\title{
4 Unternehmensnahe Stiftungen als politische Bildungsakteure
}

\author{
»Es ist ja nicht immer klar, wie viel Stif- \\ tungen mit Unternehmen zu tun haben, \\ die einen ähnlichen Namen tragen.« \\ (Donsbach/Brade 2013, S. 31) ${ }^{1}$
}

\subsection{Unternehmensnahe Stiftungen als Bildungsakteure zwischen Gemeinwohl und Partikularinteressen}

\subsubsection{Unternehmensnahe Stiftungen und Partikularinteressen}

Was ist eigentlich eine Stiftung? Eine scheinbar einfache Frage, auf die es aber keine einfache Antwort gibt. Allen Stiftungen ist zwar gemeinsam, dass sie über ein Stiftungskapital verfügen, aber dennoch verbirgt sich hinter dem Label »Stiftung « ein Spektrum an höchst unterschiedlichen Rechtsformen: Sei es die Stiftung des bürgerlichen Rechts oder die $\mathrm{GmbH}$ oder der Verein. ${ }^{2}$ Stiftungen können also sehr unterschiedlich ausgestaltet sein und im Prinzip ist jede einzelne Stiftung eine eigene Konstruktion, ein Hybrid. Neben den Rechtsformen können auch funktionale Stiftungsarten identifiziert werden. So gibt es unter anderem kirchliche, parteinahe oder eben unternehmensnahe Stiftungen - sie können in allen Rechtsformen organisiert werden sowie mit weiteren Stiftungen und GmbHs verbunden sein.

Betrachtet man diese hier nur angedeutete Vielfalt an Stiftungen, so überrascht und irritiert es, dass in bestehenden Untersuchungen $\mathrm{zu}$ Stiftungen oftmals nur allgemein und wenig differenziert von »Stiftungen « gesprochen wird. ${ }^{3}$

1 Der Kontext des Zitats wird zu Beginn des Kapitels 4.1.3 in dieser Arbeit erläutert.

2 Vgl. dazu ausführlich die Aufsätze im Kapitel »Stiftungsrecht «bei Strachwitz/Mercker 2005. Der Jurist Peter Lex führt in seinem Aufsatz aus: „Grundzüge des Stiftungsrechts ausfindig zu machen, ist ein schwieriges Unterfangen. Wer unter Stiftungsrecht die Rechtsmaterie versteht, die sich auf Stiftungen bezieht, wird an jeder Ecke unseres Rechtssystems fündig. Bundesrecht und Landesrecht, Verfassungsrecht, privates und öffentliches Recht, Kirchenrecht und Steuerrecht begleiten die Stiftungen von ihrer Errichtung bis zu ihrer Aufhebung. (Lex 2005, S. 205)

3 Zum Beispiel nennt die europäische Studie über »European Foundations for Research and Innovation« eine solche Differenzierung zwar, benutzt sie aber kaum als Analysekategorie (vgl. Euro- 
Die Vielfalt an Stiftungen - und somit auch an unternehmensnahen Stiftungstypen - wird überdeckt von einer Zahl: 94 Prozent. So hoch ist der Anteil der Stiftungen, die in Deutschland als gemeinnützig anerkannt sind (vgl. Anheier et al. 2017a, S. 10). Doch die Gemeinnützigkeit ist eine steuerrechtliche Kategorie, die nichts über gesellschaftliche und politische Funktionen von Stiftungen aussagt. ${ }^{4}$ Genau diese aber interessieren aus politikwissenschaftlicher Perspektive, in der Akteure und ihre Rolle im politischen Prozess analysiert werden. Im Hinblick auf die Frage "who or what makes things happen or prevents things from happening (Roelofs 2015, S. 657) scheinen Politikwissenschaftler*innen Stiftungen nur wenig Relevanz zuzuschreiben - in Deutschland m.E. noch weniger als in den USA. ${ }^{5}$

Die Arbeit großer US-amerikanischer »corporate foundations « wurde und wird an den Beispielen der Rockefeller Foundation, Ford Foundation und Carnegie Foundation (vgl. Arnove/Pinede 2007) bzw. in jüngerer Zeit am Beispiel der Stiftungen der Koch-Brüder (vgl. Mayer 2016) oder der Gates Foundation (vgl. McGoey 2016) kritisch untersucht. Dabei besteht kaum ein Zweifel am Erfolg großer US-Stiftungen, innen- wie außenpolitisch gezielt zu wirken und partikulare Interessen als Gemeinwohlinteressen zu verallgemeinern (vgl. Roelofs 2003, 2015; Arnove 1980; Arnove/Pinede 2007).

Demgegenüber gibt es in Deutschland bislang nur vereinzelte Studien zum Wirken von unternehmensnahen Stiftungen. In die Kritik gerieten sie zum Beispiel aufgrund einer marktliberal-ideologischen Ausrichtung politischer und ökonomi-scher Bildungsangebote, die von unternehmensnahen Stiftungen ko-finanziert werden (vgl. Möller/Hedtke 2015), oder aufgrund des Wirkens der Bertelsmann Stiftung als politischer Think-Tank (vgl. Wernicke/Bultmann 2010). Zuletzt veröffentlichte Matthias Holland-Letz (2015) eine kleine Studie mit Beispielen zur Überschneidung von Geschäftsinteressen und Stiftungsarbeit. Der Wirtschaftswissenschaftler Christian Kreiß hat die »Vernetzung von Industrie und Forschung in der deutschen Wissenschaft « untersucht und erwähnt, »dass sich viele Stiftungen als unabhängig deklarieren, in Wirklichkeit jedoch industrienahe Partikularinteressen vertreten « (Kreiß 2015, S. 178f.). »Wie konzernfreundliche Partizipation gestiftet wird « hat Thomas Wagner analysiert: Er verweist dabei auf die Rolle von unternehmensnahen Stiftungen wie der BMW Stiftung Herbert Quandt und der Bertelsmann Stiftung (vgl. Wagner 2013, S. 121-129; ausführlich dazu: Kapitel 4.2.2).

pean Commission 2015a). Auch die jüngst erschienene, umfassende Stiftungsstudie von Anheier et al. betrachtet unternehmensnahe Stiftungen nicht gesondert (vgl. Anheier et al. 2017a, b, c).

4 So betont Hanke: »Aus dieser juristischen Zweckbestimmung läßt sich weder der wirtschaftliche noch der politische Inhalt der Stiftungen ersehen. (Hanke 1971, S. 5) Auch Höhne/Schreck heben die steuerrechtliche Definition von Gemeinnützigkeit hervor (vgl. Höhne/Schreck 2009, S. 122).

5 Roelofs stellt für die USA fest, dass corporate foundations ein von Politikwissenschaftler*innen zu wenig beachteter Forschungsgegenstand seien: »Political scientists hardly ever take notice of either the official or critical literature about philanthropy and its spawn.« (Roelofs 2003, S. ix) 
Die These der politischen Funktion von unternehmensnahen Stiftungen belegt z.B. die detaillierte Untersuchung von Thomas Schuler über die Bertelsmann Stiftung (vgl. Schuler 2010): Die Stiftung nutzt den gemeinnützigen Satzungszweck »Förderung der Wissenschaft« dazu, um Studien und Policy Papers zu gesellschaftspolitischen Themen zu erarbeiten und diese öffentlichkeitswirksam, z.B. über Podien, Fachkongresse oder Kampagnen, in die Diskussion einzubringen. Auch hat die Stiftung ein breites und hochkarätiges politisches Netzwerk aufgebaut, in dem zahlreiche Spitzenpolitiker"innen - bis zu Bundeskanzler*in oder Bundespräsident"innen - zu finden sind. Schulers Analyse verweist zudem auf die politische Orientierung der Stiftung, die sich zum Beispiel im Rahmen der sogenannten Hartz-IV-Reformen u.a. mit wissenschaftlichen Expertisen für den Umbau des Sozialstaates einsetzte (vgl. Schuler 2010, S. 101ff.).

Der Satzungszweck Wissenschaft ermöglicht es Stiftungen folglich, sich als Think-Tank zu profilieren und Politikberatung zu betreiben - steuerbegünstigt und damit subventioniert von Steuerzahler*innen, wie Kritiker*innen der Bertelsmann Stiftung seit Jahren bemängeln (vgl. Lindner/Krämer/Priehn 2009; zit.n.: Schuler 2010, S. 268). Bei der Bertelsmann Stiftung wird bis heute auf den Nutzen der Stiftungsarbeit (besonders jener im Bildungsbereich) für den Bertelsmann-Konzern verwiesen. So stand sie 2015 wieder im Zentrum der (medialen) Kritik, weil sie sich - genau wie die Deutsche Telekom Stiftung - im Bereich Digitalisierung neu ausrichtete, während das dazugehörige Unternehmen Bertelsmann AG (bzw. Deutsche Telekom AG) dies auch tut (vgl. Füller 2015; Kapitel 4.2.1). Dabei ist hervorzuheben, dass die Bertelsmann Stiftung ausschließlich operativ tätig ist, das heißt, keine Projekte anderer Institutionen oder Initiativen fördert; andere Stiftungen sind dagegen auch fördernd oder sogar ausschließlich fördernd tätig.

Neben der politischen Funktion von unternehmensnahen Stiftungen verweisen US-amerikanische wie deutsche Autor*innen auf deren ökonomische Funktion (vgl. Arnove 2007; Hanke 1971). Diese besteht zunächst vor allem in umfangreichen Steuererleichterungen. Je nach Stiftungskonstruktion werden Körperschafts, Erbschafts- oder Gewerbesteuern eingespart. ${ }^{6}$ Im Hinblick auf den Satzungszweck ,Förderung von Bildung sind weitere ökonomische Funktionen zu ergänzen, z.B. die Ausbildung des eigenen betrieblichen Nachwuchses (vgl. u.a. Hanke 1971, S. 50; Holland-Letz 2015, S. 54ff.).

Die sogenannte CSR-Stiftung, bei der die Stiftung ihr Vermögen vom Unternehmen erhält, erfüllt für das Unternehmen die Funktion, mit gemeinnützigen

6 Die Familie Bertelsmann hat 2009 geschätzte 4,62 Milliarden Euro Erbschaftssteuer »durch die Stiftungskonstruktion gespart« (Schuler 2010, S. 223). Auch bei der Gründung der USamerikanischen Ford Foundation 1936 soll »die Vermeidung der Erbschaftssteuern in Höhe von über 300 Millionen ausschlaggebend« (Hanke 1971, S. 14) gewesen sein. 
Aktivitäten die "gesellschaftliche Verantwortung « des Unternehmens zu zeigen ${ }^{7}$ und ist dementsprechend in die Unternehmenskommunikation eingebunden (vgl. Junck 2007, S. 14). Als Teil von CSR gilt auch für sie das Credo aller CSR-Aktivitäten: »Nur wenn auch unternehmensintern das Engagement in seinem betrieblichen Nutzen unmittelbar einleuchtet, wird das auf lange Sicht die notwendige breite Unterstützung erfahren im Unternehmen.« (Hardtke/Kleinfeld 2010, S. 246)

Folgt man diesen Ausführungen aus der Fachliteratur, dann gehört die Verknüpfung der unternehmerischen mit gemeinnützigen Interessen somit zur offziellen Funktion von CSR-Stiftungen. Bei dieser Stiftungsform ist es deshalb auch üblich, dass hochrangige aktuelle und ehemalige Unternehmensrepräsentant*innen in den Stiftungsgremien vertreten sind. ${ }^{8}$ Das Beispiel der CSR-Stiftungen verweist damit recht anschaulich auf die Notwendigkeit, den Einfluss von Unternehmen auf Stiftungen genauer zu untersuchen - zumal diese »in den letzten Jahren die bevorzugte Erscheinungsform der Stiftung in einem unternehmerischen Umfeld [sind] (Habighorst/Seltenreich 2007). Bisher liegen aber nur Untersuchungen vor, die vor allem die umgekehrte Einflussnahme fokussieren, nämlich von Stiftungen auf Unternehmen. Hier sind die Studien der Wirtschaftswissenschaftler Marc Eulerich und Martin K. Welge zu nennen, die Unternehmensträgerstiftungen, bei denen Stiftungen Anteile am Unternehmen halten, analysiert haben (vgl. Eulerich/Welge 2011; Eulerich 2016).

Die enge Unternehmensverbundenheit von CSR-Stiftungen und das sich daraus ergebende Spannungsverhältnis von Gemeinwohl- und Partikularinteressen werden in der (deutschen) Forschungsliteratur bisher nur vereinzelt thematisiert. ${ }^{9}$ Der Bundesverband Deutscher Stiftungen (BDS) hat 2010 zudem »Zehn Empfehlungen für gemeinnützige Unternehmensstiftungen« verabschiedet (Bundesverband Deutscher Stiftung o.J.a). Diese Empfehlungen lassen jedoch viel Raum für Interpretation: Z.B., wenn gefordert wird, eine Unternehmensstiftung solle »vom

7 Junck schreibt dazu: »Errichtet ein Unternehmen eine gemeinnützige CSR-Stiftung, zeigt es öffentlich, dass ihm gesellschaftliche Verantwortung wichtig ist. (Junck 2007, S. 27)

8 Besonders bemerkenswert ist m.E. der Fall der L'Oréal-Unternehmensstiftung, bei der der Stiftungsvorsitzende auch der Konzernvorsitzende von L'Oréal Deutschland ist (vgl. L'Oréal 2017). Auch ist ein Vorstandsmitglied der Deutschen Telekom AC Mitglied des zweiköpfigen Vorstands der Deutschen Telekom Stiftung (vgl. Deutsche Telekom Stiftung 2017).

9 So hat beispielsweise Jörg Ultsch untersucht, wie die zehn Empfehlungen für gemeinnützige Unternehmensstiftungen des BDS von Unternehmensstiftungen eingeschätzt werden (vgl. Ultsch 2011). Zu CSR-Aktivitäten (in Deutschland) allgemein gibt es bisher kaum sozialwissenschaftliche Analysen: 2011 veröffentlichte Fifka überhaupt erst einmal eine »Bestandsaufnahme der Aktivitäten von Corporate Citizenship in Deutschland [...] (Fifka 2011, S. 7). Auch gibt es Handbücher, die jedoch eher praxisorientiert sind und sich an potenzielle Stiftungsgründer/innen und Unternehmen richten oder Sammelbände, in denen kaum politikwissenschaftliche Analysen zu finden sind (vgl. u.a. Backhaus-Maul et al. 2010; Schneider/Schmidpeter 2015; Hardtke/Kleinfeld 2010). 
Stifterunternehmen möglichst unabhängig sein« (ebd.). Zudem sind die Standards nicht verpflichtend - auch nicht für die Stiftungen, die Mitglied im BDS sind.

Der Konflikt von Gemeinwohl- und Partikularinteressen ergibt sich jedoch nicht nur aus der ökonomischen Funktion von Unternehmensstiftungen - als einem speziellen Typ der unternehmensnahen Stiftung -, sondern dieser Konflikt ist auch bei den sogenannten sunternehmensverbundenen Stiftungen $\mathrm{zu}$ finden, bei denen die Stiftungen Anteile an den Unternehmen halten (für diesen Stiftungstypen gibt es unterschiedliche Begriffe, vgl. Ausführungen weiter unten). Diese können als »konzerneigene Bank [...] (Hanke 1971, S. 19) fungieren oder - wie Schuler zuspitzt - als »Sparbüchse« (Schuler 2010, S. 279), da mit ihnen Gewinne im Unternehmen gehalten werden können. Dementsprechend können die Ausschüttungen an die Stiftung gering ausfallen. ${ }^{10}$ Diese Tatsache sorgte in den USA für Stiftungsreformen, die Stiftungen >nur noch 20 Prozent Anteile an einem Unternehmen erlauben. In Deutschland gibt es demgegenüber bisher keine Beschränkungen. Darüber brachte die Politikwissenschaftlerin Annette Zimmer im Kontext der Debatte um eine Reformierung der Stiftungsgesetzgebung ihre Verwunderung zum Ausdruck:

»Wir sind eines der wenigen Länder, wo es möglich ist, dass Unternehmen und Stiftungen in der Weise verkoppelt sind, wie es zum Beispiel bei der Bosch Stiftung oder bei der Bertelsmann Stiftung der Fall ist. In den USA ist das verboten, und ich glaube, es gibt auch gute Cründe, dass man diese beiden Bereiche trennt. [...]« (Zimmer; zit.n.: Schuler 2010, S. 243)

Ein Vorschlag für eine Stiftungsreform sah 1997 den Grundsatz vor, dass Stiftungen keine Zwecke verfolgen dürften, die »auf einen wirtschaftlichen Geschäftsbetrieb gerichtet sind « (Deutscher Bundestag 1997, S. 3). Gegen die Reformvorschläge setzten sich die Bertelsmann Stiftung und auch der Bundesverband Deutscher Stiftungen als Interessenvertretung deutscher Stiftungen erfolgreich zur Wehr: Die Reform verschwand von der politischen Agenda. ${ }^{11}$ Das damals verhandelte Argument, dass Stiftungen keine Geschäftsinteressen verfolgen sollten, wirkt vor dem heutigen Boom und zum Beispiel der steuerlichen Begünstigung von CSR-Stiftungen fast schon antik. Denn CSR-Stiftungen sind als Botschafter für ihre Unternehmen - hochoffiziell und für jeden auf Anhieb ersichtlich - mit den Geschäftsinteressen ihrer Eigentümer verbunden.

Sowohl die politischen als auch die ökonomischen Funktionen von unternehmensnahen Stiftungen verweisen auf Partikularinteressen, die mit der Gründung

10 Schuler problematisierte dies 2010 für die Bertelsmann Stiftung (vgl. Schuler 2010, S. 217), Holland-Letz 2015 für die Robert Bosch Stiftung (vgl. Holland-Letz 2015, S. 131ff.).

11 In diesem Zusammenhang verweist Schuler auch auf die starken Lobbyverbände im Stiftungsbereich (vgl. Schuler 2010, S. 233ff.). 
einer als gemeinnützig anerkannten, unternehmensnahen Stiftung verknüpft werden können. Damit werden die dem offiziellen gemeinnützigen Stiftungszweck entsprechenden Aktivitäten keineswegs in Abrede gestellt, aber es erscheint dringend erforderlich, eine realistischere Analyse der Multifunktionalität von unternehmensnahen Stiftungen an die Stelle einseitiger Postulate im Sinne der Gemeinwohlorientierung zu setzen.

Dies wird aber meist nur an Einzelfällen wie der Bertelsmann Stiftung gezeigt. Ist die Bertelsmann Stiftung aber vielleicht nur ein »schwarzes Schaf«? Oder werden Stiftungen und gemeinnützige Stiftungszwecke (wie etwa politische Bildung) von vielen unternehmensnahen Stiftungen zur erweiterten politischen Einflussnahme genutzt? Welche Formen kann diese erweiterte Einflussnahme annehmen ? $^{12}$ Helmut Anheier et al. haben 2017 die Ergebnisse eines großen Forschungsprojekts zum Stiftungswirken in unterschiedlichen Bereich (Bildung, Wissenschaft, Soziales, Kultur) publiziert, jedoch werden auch hier unternehmensnahe Stiftungen nicht gesondert betrachtet (vgl. Anheier et al. 2017a, b, c). Wie bereits eingangs (vgl. Kapitel 1) erwähnt, gibt es bisher keine einzige Studie, die das Wirken unternehmensnaher Stiftungen in Deutschland in einem bestimmten Politik- oder Themenfeld untersucht. Lediglich die Studie von Horst Hanke aus dem Jahr 1971 hat einen (aber keinen ausschließlichen) Schwerpunkt auf unternehmensnahe Stiftungen, wenn sie unter anderem nach der »Rolle der Stiftungen in der staatsmonopolitischen Wissenschaftspolitik« fragt (vgl. Hanke 1971). Eine systematisierende Untersuchung mit dem Bezug zu einem bestimmten Bereich steht aktuell somit (noch) aus.

Die Wirtschaftswissenschaftler Eulerich/Wagner konstatieren in ihrer Studie über den Einfluss von Stiftungen auf Unternehmen großen Bedarf an Grundlagenforschung (vgl. Eulerich/Welge 2011, S. 93ff.). Bislang wird der mitunter sehr enge Zusammenhang von Stiftungen und Unternehmen kaum analysiert (vgl. ebd., S. 9), auch wenn Eulerich mit seiner Studie »Stiftungsverbundene Unternehmen in Deutschland« von 2016 einen wesentlichen und aktuellen Beitrag leistet (vgl. Eulerich 2016). Die Untersuchung umfasst u.a. eine qualitative Mehrfallstudie zu stiftungsverbundenen Unternehmen, in der auch personelle Verflechtungen zwischen

12 In den USA werden auch als progressiv gehandelte Vorhaben unternehmensnaher Stiftungen kritisch befragt: So gehen Arnove/Pinede in Bezug auf die Ford Foundation und die Stiftungen von Rockefeller und Carnegie der Frage nach, »[...] how the big three foundations over the last two decades have attempted to respond to national and international events in a progressive way; and yet, how they might also still be considered representative of what Fisher (1980) has called a >sophisticated conservatism, $<$ [sic!] supporting changes that help to maintain and make more efficient an international system of power and privilege. Although these foundations claim to attack the root causes of the ills of humanity, they essentially engage in ameliorative practices to maintain social and economic systems that generate the very inequalities and injustices they wish to correct. «(Arnove/Pinede 2007, S. 392f.; Fisher 1980) 
Stiftungen und Unternehmen analysiert werden, die prinzipiell eine Einflussnahme nicht nur von Stiftung auf das Unternehmen, sondern auch umgekehrt zulassen (vgl. ebd., S. 317ff.). ${ }^{13}$

Ein Resultat der fehlenden Grundlagenforschung ist der feststellbare Mangel an konzeptioneller und begrifflicher Klarheit. In der Fachliteratur gibt es keine einheitliche Verwendung von Begriffen: Zum Beispiel wird die Robert Bosch Stiftung in einer Studie des BDS als Unternehmensbeteiligungsstiftung oder von dem Wirtschaftswissenschaftler Matthias Fifka als Beteiligungsträgerstiftung bezeichnet, bei Eulerich wird sie als Unternehmensträgerstiftung analysiert (vgl. Junck 2007, S. 14; Fifka 2011, S. 121; Eulerich 2016, S. 322ff.). Angesichts der fehlenden Grundlagenforschung wundert es auch nicht, dass selbst der BDS als größte Interessenvertretung deutscher Stiftungen keine Angaben darüber machen kann, wie viele Stiftungen unter den Begriff >unternehmensnah، fallen bzw. was eigentlich eine unternehmensnahe Stiftung ist.

Zwar hat der BDS bereits im Jahr 2007 eine Studie zu unternehmensnahen Stiftungen beauftragt, in der nach Definition, Stiftungstypen und Anzahl von unternehmensnahen Stiftungen gefragt wird und für die ein erster Datensatz zusammengestellt wurde, in welchem drei Stiftungstypen unterschieden wurden (Unternehmensträger-, Unternehmensbeteiligungs- und Unternehmensstiftung). ${ }^{14}$ Weil die Kategorie »unternehmensnah« damals aber zu »unkonkret« gefasst worden sei, stellt der BDS den Datensatz für weitere wissenschaftliche Analysen nicht zur Verfügung. ${ }^{15}$ Der Datensatz umfasst rund 1500 Stiftungen, wobei auch die rund 600 Sparkassenstiftungen aufgenommen wurden, die aber aus Sicht des BDS einen "Sonderfall« darstellen (Junck 2007, S. 32). ${ }^{16}$

Um zu klären, welche Stiftungen aus welchen Gründen als >unternehmensnah gelten können, werden deshalb im Folgenden in gebotener Kürze unternehmensnahe Stiftungstypen erläutert und dabei auch die unterschiedlichen Fachmeinungen angeführt, um die Umstrittenheit der Begriffe zu verdeutlichen. Während in der US-Fachliteratur der Begriff >corporate foundation` gebraucht wird, werden in dieser Studie nachfolgend nicht die Begriffe >Unternehmensstiftungく oder >unternehmensverbundene Stiftungen als Oberbegriffe genutzt. Denn anders als im allgemeinen Sprachgebrauch stellen diese in der Fachliteratur Begriffe für bestimm-

13 Auf einige Ergebnisse dieser Studie zur Robert Bosch Stiftung wird in Kapitel 4.3.1 eingegangen.

14 Die Studie stellt damit einen Überblick über die zahlreichen Organisationsformen bereit, jedoch keine Analyse der Stiftungsstrukturen und -funktionen unternehmensnaher Stiftungen.

15 Diese Aussage ist einem Gespräch mit einer BDS-Mitarbeiterin im August 2015 entnommen. Aufgrund der Schwierigkeit mit der Kategorie »unternehmensnah« ist die Studie auch nicht mehr veröffentlicht.

16 Die 600 Sparkassenstiftungen gehörten zu »den fünf kapitalstärksten Unternehmensstiftungen Deutschlands«, würde man von ihrem Gesamtvermögen in der Höhe von 1,3 Mrd. Euro ausgehen (Junck 2007, S. 32). 
te Typen dar, die in der vorliegenden Studie unter dem Oberbegriff sunternehmensnahe Stiftungen zusammengefasst werden. Dies geschieht in begrifflicher Anlehnung an die erwähnte Typologie unternehmensnaher Stiftungen in der BDSStudie. Die Autorin Sibylle Junck unterscheidet drei Typen; sie stützt sich dabei auf die bestehende Fachliteratur und verschiedene (meist vom BDS initiierte) Fachtagungen, die rund um das Thema >Stiftung und Unternehmen< stattfanden (vgl. Junck 2007, S. 9-14):

- die Unternehmensträgerstiftung, bei der die Stiftung selbst am Markt agiert, Unternehmens- und Stiftungszweck fallen bei diesen Stiftungen zusammen (vgl. ebd., S. 14);

- die Unternehmensbeteiligungsstiftung, die Anteile am Unternehmen hält, bei der aber Stiftungs- und Unternehmenszweck getrennt sind (vgl. ebd.);

- die Unternehmensstiftung (CSR-Stiftung), die ihr Vermögen vom Unternehmen erhält und in der Regel keine Unternehmensanteile besitzt, wie Junck ausführt (vgl. ebd.).

Eulerich weist auf das Problem der Ungenauigkeit des Begriffs der Unternehmensträgerstiftung hin: Was eine solche »explizit konstituiert, ist in der Wissenschaft nicht definiert. (Eulerich 2016, S. 109) Er arbeitet mit dem Begriff der Unternehmensträgerstiftung, wobei man analog zur bestehenden Fachliteratur zwischen Unternehmensträgerstiftungen im engen und weiten Sinn unterscheiden könne (vgl. ebd., S. 110). ${ }^{17}$ Analog zu seinen Ausführungen kann man die ersten beiden unternehmensnahen Stiftungstypen in Juncks Studie (»Unternehmensträgerstiftung « und »Unternehmensbeteiligungsstiftung«) unter diesem Begriff subsumieren. Denn unter Unternehmensträgerstiftungen im engen Sinn würden in der Wissenschaft Unternehmen verstanden, »die in der Rechtsform der Stiftung geführt werden (ebd.), ${ }^{18}$ wobei die Stiftung »unmittelbarer Inhaber des Unternehmens und [...] somit persönlich haftender Gesellschafter einer OHG oder KG « sei (ebd.). ${ }^{19}$

17 An anderer Stelle spricht er von unternehmensverbundenen Stiftungen mit »unmittelbare[n] und mittelbaren Verbindungen «zum Unternehmen: Bei erstgenannten »hat das Unternehmen selbst die Rechtsform der Stiftung, beispielsweise als Stiftung \& Co. KC. Deutlich häufiger anzutreffen ist der mittelbare Fall: Eine Stiftung hält Anteile an einem Unternehmen. Die Beteiligung kann unterschiedliche Formen annehmen: von der Minderheitsbeteiligung über Beteiligungen, die eine Sperrminorität erlauben, bis hin zum Mehrheitseigner und alleinigen Eigner.« (Eulerich; zit.n.: Bundesverband Deutscher Stiftungen 2013b, S. 40)

18 Eulerich bezieht sich hier auf: Müller-Faßbender 1994, S. 99; Brandmüller/Lindner 2005, S. 68f.; Trappe 2010, S. 146.

19 Dies belegt Eulerich mit dem Verweis auf: Werthern 1986, S. 98; Müller-Faßbender 1994, S. 99; Wigand et al. 2011, S. 35. Die hier als Unternehmensträgerstiftung im engen Sinn beschriebene Form hätten jedoch nur wenige Stiftungen in Deutschland, z.B. die Carl-Zeiss-Stiftung bis zur Änderung ihrer Stiftungsstruktur im Jahr 2004 (vgl. Eulerich 2016, S. 110). 
Häufiger kämen jedoch »Unternehmensträgerstiftungen im weiten Sinn bzw. mittelbare Unternehmensträgerstiftungen« (ebd.) vor. Dazu gehören »all jene Stiftungen, die an einem Unternehmen beteiligt sind « (ebd.). ${ }^{20}$ Das Unternehmen ist hier in einer »eigene[n], von der Stiftung losgelöste[n] Rechtsform (Personen- oder Kapitalgesellschaft)« organisiert, die Stiftung kann wiederum

»als Gesellschafter Einfluss auf den eigentlichen Unternehmensträger ausüben. Demnach wird das Unternehmen nach erwerbswirtschaftlichen Grundsätzen geführt, während die daraus resultierenden ausgeschütteten Cewinne teilweise oder ganz der Stiftung zufließen.«(Ebd.) ${ }^{21}$

Bei den Unternehmensträgerstiftungen im weiten Sinn gibt es weiterhin unterschiedliche Typen, bei denen m.E. zu fragen wäre, ob diese unterschiedlichen Gestaltungsformen die Ausrichtung der gemeinnützig anerkannten Stiftungsarbeit beeinflussen. So wird zwischen Stiftungen unterschieden, die »unternehmerisch (Beteiligungsträgerstiftung) oder rein vermögensverwaltend (Kapitalfondsträgerstiftung) aktiv« (ebd.) sind. ${ }^{22}$ Laut Eulerich sind »die Kriterien zur Abgrenzung « dieser beiden Typen »in Literatur und Rechtsprechung umstritten « (ebd., S. 111). ${ }^{23}$

Basierend auf diesen beiden einzigen aktuelleren Studien $\mathrm{zu}$ unternehmensnahen Stiftungstypen von Eulerich (2016) und Junck (2007) können m.E. somit zunächst zwei Stiftungstypen identifiziert werden, die (vergleichsweise unumstritten) als unternehmensnah gelten dürfen:

\section{- Unternehmensstiftung (auch: CSR-Stiftung)}

- Unternehmensträgerstiftungen im engen und weiten Sinne, auch als unternehmensverbundene Stiftungen bezeichnet

Auch der Bundesverband Deutscher Stiftungen unterscheidet in einer »Stiftungstypologie« zwischen diesen beiden Typen: Unternehmensstiftungen, »für die das Unternehmen das Stiftungsvermögen und gegebenenfalls laufende Mittel bereitstellt", und unternehmensverbundenen Stiftungen, die "Anteile an Unternehmen [halten] oder [...] selbst ein Unternehmen [betreiben]« (Bundesverband Deutscher Stiftungen 2013b, S. 125f.).

$\mathrm{Da}$ aber ungeklärt ist, »[z]u welchem prozentualen Anteil ein Unternehmen mit einer Stiftung verbunden sein muss, um als stiftungsverbunden zu gelten [...]« (ebd., S. 37), und es dazu keine »eindeutige juristische Definition« gibt, wie Eulerich in einem Interview mit dem BDS betont (Eulerich; zit.n.: Bundesverband

20 Diese würden »auch als Beteiligungsträgerstiftungen bezeichnet« (Eulerich 2016, S. 110). Eulerich bezieht sich hier auf Schwake 2008, S. 6.

21 Eulerich bezieht sich hier auf: Trops 1970, S. 367; Werthern 1986, S. 97.

22 Eulerich bezieht sich hier auf die Angaben in: Berndt 2003, S. 522f.; Schwake 2008, S. 7.

23 Eulerich bezieht sich auf: Berndt 2003, S. 526; Trappe 2010, S. $121 \mathrm{f}$. 
Deutscher Stiftungen 2013b, S. 40), sind unternehmensverbundene Stiftungen als solche nicht immer leicht zu identifizieren. Schaut man sich einige gemeinnützige private Stiftungen im Bildungsbereich an, so fällt auf, dass diese zwar Unternehmensanteile halten und insofern zu Unternehmensträgerstiftungen im weiten Sinn (nach obiger Definition) bzw. als eine mittelbare unternehmensverbundene Stiftung gelten könnten, aber als solche nicht gleich erkenntlich sind. ${ }^{24}$

Darüber hinaus gibt es viele private Stiftungen, die kaum Angaben zu ihrem Vermögen machen und daher nicht bekannt ist, ob sie Unternehmensanteile halten und als unternehmensverbundene Stiftung gelten dürfen. Dies lässt sich am Beispiel der Stiftung Mercator verdeutlichen, die sich auch auf dem Feld der politischen Bildung engagiert: Sie wurde von der Familie Schmidt gegründet, die zu den Hauptanteilseignern der Metro Group gehört, und ist »mit erheblichen finanziellen Mitteln« (Stiftung Mercator 2017) aus dem Vermögen der Familie ausgestattet. Mehr Information gibt die Stiftung über die Herkunft ihres Vermögens von rund 279 Millionen Euro nicht preis (dies. 2015, S. 17). Folglich kann auch über die »Unternehmensnähe« der Stiftung Mercator nur spekuliert werden, da nicht bekannt ist, ob und in welchem Umfang Aktien und Unternehmensbeteiligungen zum Teil des Stiftungsvermögens gehören. Aufgrund fehlender Transparenzstandards (vgl. Kapitel 4.1.3) ist oft nicht bekannt, wie sich das Vermögen von Stiftungen genau zusammensetzt; Aktien und Unternehmensbeteiligungen gehören dabei nicht selten zu dem privaten Vermögen von Unternehmer*innen und damit auch zum Stiftungsvermögen vieler privater Stiftungen.

Es wird also deutlich, dass im Falle von sprivaten Stiftungen Recherchebedarf besteht, um Art und Umfang möglicher Unternehmensverbindungen zu ermitteln. Solange sie ihre Vermögensstruktur nicht offenlegen, können Stiftungen, wie die Stiftung Mercator, m.E nicht als eindeutig nicht-unternehmensnah gelten. Dabei sei auch noch einmal betont, dass nicht klar ist, ob und inwiefern das Halten von Unternehmensanteilen die weiteren Stiftungsstrukturen und -arbeit beeinflusst. Dies müsste z.B. anhand von Fallstudien untersucht werden.

Die auf dem Feld der politischen Bildung agierende Stiftung Mercator, ZEITStiftung oder Gemeinnützige Hertie Stiftung könnten auch unter dem Begriff der »Unternehmer-Stiftung« geführt werden, zu denen Junck Stiftungen zählt, »deren

24 Hier wäre zum Beispiel die Joachim Herz Stiftung zu nennen, die auf dem Feld der ökonomischen und politischen Bildung tätig ist (vgl. Kapitel 4.2) und die oft als `private Stiftung` geführt wird (vgl. u.a. ihre Nennung im Band »Private Stiftungen als Partner der Wissenschaft«, Bundesverband Deutscher Stiftungen 2013a). Es gibt auch Beispiele von im Bildungsbereich tätigen Stiftungen, die ihre Aktienanteile an Croßkonzernen gar nicht auf der Stiftungs-Website vermerken: So z.B. die Dietmar Hopp Stiftung, die von einem der Mitgründer des SoftwareKonzerns SAP, Dietmar Hopp, ins Leben gerufen wurde. Ihr »Stiftungsvermögen besteht überwiegend aus SAP-Aktien, die Dietmar Hopp aus seinem privaten Besitz eingebracht hat.«(Bundesverband Deutscher Stiftungen o.J.b) 
Kapital aus dem Privatvermögen eines Unternehmers besteht « (Junck 2007, S. 13). ${ }^{25}$ Diese Kategorie ist jedoch u.a. dahingehend als ungenau zu bezeichnen, als dass weitere Verbindungen zum Unternehmen (z.B. Unternehmensanteile) wenig Beachtung finden, weshalb nachfolgend mit ihr nicht gearbeitet wird. Aus einer politikwissenschaftlichen Perspektive auf das Thema unternehmensnahe Stiftungen im Spannungsfeld von Partikular- und Gemeinwohlinteressen wäre es insgesamt sinnvoll, verschiedene Dimensionen der Unternehmensverbundenheit zu differenzieren und ihren jeweiligen Einfluss auf die Stiftungsarbeit zu analysieren.

\subsubsection{Politischer Einfluss und institutionelle Strukturen}

In der sallgemeinen Stiftungsforschung, die oftmals eben nicht zwischen den unterschiedlichen Stiftungen bzw. Stiftungsformen unterscheidet, wird oft betont, dass Stiftungen nicht sehr einflussreich seien - zu klein sei ihr Budget für struktu-

25 Junck fasst diesen Stiftungstyp sogar als »Unternehmensstiftung i.w.S.« (Junck 2007, S. 14). Sie bezieht sich dabei auf »eine Diskussion über Begrifflichkeiten« bei einer Tagung der Arbeitsgruppe Unternehmensverbundene Stiftungen im Jahr 2007. Dort sei es »Tenor « gewesen, »sich an der Praxis zu orientieren und bei der Kategorisierung des Typs der Unternehmensstiftung (i.w.S.) als Kriterien die Person des Stifters und die Vermögensquelle zu berücksichtigen« (ebd., S. 13). Obwohl Junck die »Unternehmer-Stiftung «zur»Unternehmensstiftung i.w.S. «zählt, steht bei der darauffolgenden Beschreibung nur noch die »Unternehmensstiftung (CSR-Stiftung) « im Titel (ebd., S. 14). Dies erweckt den Eindruck, als bedürfe es keiner näheren Betrachtung der >Unternehmer-Stiftung «. M.E. unterscheidet sich die sogenannte Unternehmer*innen-Stiftung substanziell von dem Typ der Unternehmensstiftung, die direkt von einem Unternehmen errichtet wird. Anders als bei Unternehmensstiftungen kommt das Vermögen i.d.R. nicht direkt aus dem Unternehmen, sondern stammt aus dem Privatvermögen von Unternehmer ${ }^{*}$ innen; die Stiftungen werden zudem von Privatpersonen, nicht von Unternehmen gegründet. Eine Zuordnung zu den Unternehmensstiftungen erscheint insofern nicht ausreichend differenzierend. 
relle Reformen. ${ }^{26}$ Gleichzeitig findet die hohe Konzentrierung an Stiftungskapital ${ }^{27}$ und damit potenziell einhergehende Möglichkeiten zur politischen Einflussnahme nicht ausreichend Beachtung. Der ausschließliche Blick auf das Geldvermögen kann dazu führen, dass die Steuerungskapazitäten und der Einfluss von Stiftungen im Allgemeinen und von unternehmensnahen Stiftungen im Besonderen systematisch unterschätzt werden. So sind beispielsweise Agenda Setting ${ }^{28}$ und die Herausbildung von strategischen Koalitionen zur Durchsetzung von bestimmten Orientierungen nicht - zumindest aber nicht allein - durch den Anteil der verfügbaren finanziellen Mittel in einem Arbeitsgebiet bestimmt.

Unternehmensnahe Stiftungen sind darüber hinaus als besondere Institutionen in Bezug auf Fragen des politischen Wandels zu erachten. Wie der Erziehungswissenschaftler Höhne herausgearbeitet hat (vgl. Höhne 2012, S. 247), vereinen sie meist alle komplementär wirkenden, von Pierre Bourdieu definierten Kapitalformen: Sie sind finanziell sehr gut ausgestattet und verfügen somit über ein hohes ökonomisches Kapital; sie können (über Stiftungsgremien, Veranstaltungen, Projektkooperationen usw.) ein hohes soziales Kapital, z.B. in Form von Netzwerken, oder ein hohes kulturelles Kapital erlangen, z.B. Wissen darüber, welche Regeln in einem sozialen Feld als anerkannt gelten. Aus diesen drei Kapitalien generiert sich (jeweils und besonders im Zusammenspiel) zudem ein hohes symbolisches Kapital, in Form von Reputation und gesellschaftlicher Anerkennung, das auch durch die anerkannte Gemeinnützigkeit und ggf. dem Image einer Wissenschafts-, Bildungs-

26 So wird z.B. im Germany Country Report der EUFORI-Studie eine Aussage des Geschäftsführers der Stiftung Mercator (Wolfgang Rohe) zu Wissenschaftsstiftungen in Deutschland hervorgehoben: " Science foundations provide only about $1 \%$ of public science funding' « (Rohe; zit.n.: European Commission 2015b, S. 38). Anheier et al. schätzen das »Ausgabenvolumen der deutschen Stiftungen« auf 13,1 Milliarden Euro (Anheier et al. 2017a, S. 232) und betonen: „Selbst wenn die Vermögen der deutschen Stiftungen, zu deren Größe weiterhin keine verlässlichen Angaben vorliegen, die aber grob auf 100 Milliarden Euro[...] geschätzt werden, für Außenstehende als hoch erscheinen und selbst Experten des Dritten Sektors erstaunt aufschauen lassen, so sind die daraus fließenden Summen des Ausgabenvolumen jährlich geschätzte 13,1\%. Mit anderen Worten, wenn innerhalb eines Jahres die gesamten Erträge des Stiftungsvermögens verausgabt würden, könnte dadurch der deutsche Dritte Sektor bezogen auf seine Bruttowertschöpfung von 89 Milliarden Euro für gerade einmal knapp acht Wochen finanziert werden." (Ebd., S. 233) Bei der Schätzung auf 100 Milliarden Euro wird sich bezogen auf: Rosenski 2012.

27 Anheier et al. verweisen auf die »[...] frappierende Ungleichverteilung der finanziellen Mittel im Stiftungswesen: 15 \% der deutschen Stiftungen fallen in die Kategorie der großen Dienstleister, deren Anteil am jährlichen Gesamtbudget aller Stiftungen bei $42 \%$ liegt. Die kleineren Nischenanbieter machen hingegen $29 \%$ aller Stiftungen und gerade einmal $1 \%$ des Cesamtbudgets aus. Betrachtet man nur das Budget aller operativ und gemischt tätigen Stiftungen, so liegt der Anteil der Dienstleister am Cesamtbudget sogar bei $97 \%$.« (Anheier et al. 2017a, S. 82f.)

28 Höhne/Schreck kommen zu dem Schluss, dass Stiftungen über ihre Projekte, Gesprächsrunden etc. »[...] wesentlich zur Formierung von Diskursen bei[tragen]« (Höhne/Schreck 2009, S. 124). 
oder Kulturstiftung befördert wird. Hierbei spielt auch eine Rolle, dass ressourcenstarke unternehmensnahe Stiftungen nicht nur die (oben dargestellten) verschiedenen Kapitalformen in sich vereinen können, sondern auch »ein spezieller Ort der Vermittlung besagter Kapitalien an andere Akteure« (Höhne 2012, S. 243) sind.

Ignoriert man das Zusammenspiel der Kapitalformen, das oftmals bei den ressourcenstarken unternehmensnahen Stiftung anzutreffen ist, dann bleiben Machtpotenziale von Stiftungen unerkannt, zum Beispiel der Einfluss von Machteliten, die vielleicht nicht ein gesamtes Politikfeld dominieren, aber dafür in einem Teilbereich beträchtlichen Einfluss ausüben können. ${ }^{29}$ Als Beispiel für den Bildungsbereich kann das Engagement der Deutschen Telekom Stiftung in der MINT-Förderung ${ }^{30}$ angeführt werden. Die Stiftung sieht

»ihren größten Erfolg darin, die öffentliche Aufmerksamkeit für das Thema der MINT-Bildung zu erhöhen. Die Stiftung war von Anfang an dabei als das Thema zu Beginn des letzten Jahrzehnts an Relevanz gewann. Die Stiftung sieht es auch als ihren Verdienst andere Stiftungen und Akteure mit auf das Thema gebracht zu haben. Sie ist zudem Mitgründer oder Financier wichtiger MINTInitiativen wie bei der Arbeitgeberinitiative >MINT-Zukunft schaffen<, dem Haus der Kleinen Forscher oder dem Nationalen MINT-Forum. Auch die Landschaft der mathematischen Lehrerbildung sehe ohne die Deutsche Telekom Stiftung und das von ihr initiierte Deutsche Zentrum für Lehrerbildung Mathematik deutlich anders aus: Ein Konsortium aus aktuell sieben Hochschulen erarbeitete die fachdidaktischen Grundlagen und es sei die Fachdidaktik als Community gestärkt und ein wesentlich höherer Grad an Vernetzung erreicht worden.« (Striebing 2017b, S. 57)

Problematisch an Stiftungen ist dabei, dass sie »Einfluss auf die öffentliche Ressourcenallokation « (Adloff 2010, S. 378) nehmen, obwohl sie selbst über keine - etwa den politischen Parteien oder anderen Mitgliederorganisationen vergleichbare - demokratische Legitimation und Rückbindung verfügen. Wie in der Stiftungsfachliteratur regelmäßig festgestellt wird, sind Stiftungen nicht rechenschaftspflichtig gegenüber Mitgliedern, Wähler*innen oder Anteilseigner*innen,

29 Ein bekanntes Beispiel für die These liefert die Analyse von Thomas Schuler zur Rolle der Bertelsmann Stiftung in der Hochschulpolitik (vgl. Schuler 2010, S. 138-175). Auch der Germany Country Report der EUFORI-Studie, die Wissenschaftsstiftungen untersucht, weist darauf hin, dass es einige wenige große Stiftungen gibt, »which control the majority of the financial means of the sector. These big foundations can in fact influence a certain field of research by funding either a specialised infrastructure or individuals that fit into the foundations \& focus programs." (European Commission 2015b, S. 37)

30 »MINT-Fächer ist eine zusammenfassende Bezeichnung von Unterrichts- und Studienfächern beziehungsweise Berufen aus den Bereichen Mathematik, Informatik, Naturwissenschaft und Technik.«(Wikipedia 2017a) 
wie das zum Beispiel bei einem Verein, einer Partei oder einem Unternehmen der Fall ist (vgl. u.a. Anheier/Appel 2004, S. 13). Schuler hat deshalb in Hinblick auf die Bertelsmann Stiftung und ihre politische Einflussnahme über Politikberatungstätigkeiten die berechtigte Frage aufgeworfen: »[...] wie demokratisch muss eine Stiftung sein, die Einfluss auf die Demokratie nimmt?« (Schuler 2010, S. 284)

Hinzu kommt, dass die bestehende Stiftungsforschung das Stiftungswesen als »Elitenphilanthropie« (Adloff 2010, S. 405) beschreibt und die innere Organisation von Stiftungen insgesamt eher »vordemokratisch-dynastischen Entscheidungsstrukturen« (Höhne 2012, S. 249) entspricht (was wiederum der Durchsetzung partikularer Interessen Vorschub leistet). Die Aussage des Politikwissenschaftlers Kenneth Prewitt über das US-amerikanische Stiftungswesen trifft auch auf das deutsche $\mathrm{zu}$ :

»Der Stiftungssektor ist per Definition und Cesetz weitgehend undemokratisch, denn wie sonst soll man eine wohlhabende Elite beschreiben, die steuerfreie Dollars verwendet, um ihre Vision des öffentlichen Wohls geschehen zu lassen?« (Prewitt 2011, S. 96)

Anheier/Appel gehen bei Stiftungen von »engen Verbindungen zur politischen und gesellschaftlichen Elite« (Anheier/Appel 2004, S. 10) aus und stellen fest, dass »(i)m Falle Großbritanniens und der USA [...] Stiftungen von der Elite des Landes nicht nur gegründet, sondern auch geführt und kontrolliert werden« (ebd., S. 9). Umso mehr überrascht, wie wenig die elitäre Ausrichtung von deutschen Stiftungen - zum Beispiel mit Blick auf die Stiftungsorgane und Entscheider*innen in Stiftungen - empirisch erforscht ist. Geht man von dem weitgehend anerkannten Verständnis der funktionalistischen Eliteforschung aus, so zählen zu den gesellschaftlichen Eliten »Inhaber der jeweils höchsten Positionen aus Politik [...], Verwaltung [...], Wirtschaft [...], Justiz [...], Medien [...], Wissenschaft [...], Militär [...] und Gewerkschaft [...] (Hartmann 2008, S. 10f.). ${ }^{31}$ Ob deren Anteil unter den Gremienmitgliedern in den ressourcenstarken unternehmensnahen Stiftungen in Deutschland besonders hoch ist, ist bisher nicht erforscht.

Ein Blick auf die institutionelle Binnenstruktur und Gremien von Stiftungen verrät, dass eine Stiftung nur ein einziges Organ besitzen muss: einen Vorstand, der laut Gesetz sogar nur aus mindestens einer Person bestehen muss (vgl. Wigand et al. 2015, S. 42). Die Funktion des Vorstands wird zudem unterschiedlich benannt, was die Transparenz nicht gerade erhöht. Der Vorstand »entscheidet [...] alleine über die Geschäftsführungsmaßnahmen« und »verfügt damit über einen großen Handlungsspielraum« (ebd., S. 47). Die Geschäftsführung beinhaltet die

31 Hartmann führt aus, dass »diese Auflistung von allen, das heißt auch den kritischen Eliteforschern geteilt« werde, lediglich »die Einbeziehung der Gewerkschaftsspitzen« sei »nicht unumstritten« (Hartmann 2008, S. 11). 
»[...] Verwaltung des Stiftungsvermögens und der Verwirklichung der Stiftungszwecke im Rahmen der Stiftungssatzung durch die Erträge des Stiftungsvermögens« (ebd.). Laut Gesetz darf also nur eine einzige Person über die Verwendung des Stiftungsbudgets bestimmen und ist gesetzlich nicht dazu verpflichtet, einem zweiten kontrollierenden Organ Rechenschaft abzulegen.

Oftmals ist der Vorstand um ein zweites Organ, einen Stiftungsrat, ein Kuratorium oder ggf. weitere Organe (z.B. einem Wissenschaftlichen Beirat) ergänzt. Diesen weiteren Organen wird in der Fachliteratur eine Kontrollfunktion zugesprochen, da sie unter anderem den Vorstand beraten, die Haushalts- und Wirtschaftsführung prüfen, den Vorstand be- und abrufen können (vgl. ebd., S. 49). Diese Einschätzung, dass zusätzliche Organe eine Kontrollfunktion haben, ist jedoch zu relativieren: Denn wie sie ausgestaltet sind, wird von dem*der Stifter*in festgelegt. Karl-Heinz Boeßenecker, ehemaliger Bundesgeschäftsführer der Stiftung Mitarbeit, weist deshalb auf den Widerspruch zwischen dem oftmals vertretenen Anspruch gemeinnütziger Stiftungen, Demokratie fördern zu wollen, und der eigenen Struktur hin:

»Institutionell trägt die Rechtsform der Stiftung dem von ihr vertretenen partizipativen Demokratie- und Förderverständnis nur bedingt Rechnung. Denn die Zusammensetzung der Gremien folgt unverändert der Logik der Kooptation.« (Boeßenecker 2014, S. 64) ${ }^{32}$

Hinzu kommt, dass selbst wenn es ein Kuratorium gibt, dort auch Personen aus dem Stiftungsvorstand in Personalunion vertreten sein dürfen. Komplexer wird das Feld noch dadurch, dass Stiftungen in unterschiedlichen Rechtsformen organisiert sind und die Funktionen der Organe in der Satzung festgelegt werden und von Stiftung zu Stiftung unterschiedlich ausgestaltet sein können.

In manchen Stiftungen gibt es zudem Personalunionen zwischen Stiftungsvorstand und Kuratorium oder Stiftungsorgane werden gar gleich komplett mit denselben Personen besetzt (z.B. bei der Robert Bosch Stiftung). Aus Sicht des*der Stifters*in mag das Sinn ergeben, um sich und den Nachfolgenden Einfluss zu sichern. Aus demokratietheoretischen Überlegungen dagegen erscheint die fehlende öffentliche Kontrolle über das steuerlich begünstigte Vermögen, das zu gemeinnützigen Zwecken eingesetzt werden soll, problematisch. Der Soziologe Clemens Striebing hat sich in seiner jüngst erschienenen Dissertation (2017a) mit der »Le-

32 Wenig überraschen dürfte vor diesem Hintergrund auch, dass »[a]llem Anschein nach [...] die Personalstruktur in Stiftungen ziemlich homogen [ist] « (Bundesverband Deutscher Stiftungen 2012b, S. 60). Man stößt vor allem auf »eine weiße Mittelschicht - gut (aus-)gebildet, gut situiert und sehr engagiert« (ebd.). Der BDS stellt hier einen »Nachholbedarf« im Stiftungswesen fest (ebd.). 
gitimierung von Stiftungen« u.a. im Rahmen einer Satzungsanalyse auseinandergesetzt. Auf dieser Basis befindet er,

»dass das rechtswissenschaftliche Paradigma von der seigentümerlosen Stiftung grundsätzlich auf dem Prüfstand gestellt werden sollte. Wenn die Covernance von mehr als der Hälfte der untersuchten Stiftungen auf ihre Financiers und Gründer zugeschnitten ist, kann nicht von einer wirklichen Aufgabe von Eigentümerrechten gesprochen werden.« (Ebd., S. 367)

Er konstatiert, dass mit Stiftungen den »[n]ormalen Bürgern [...] plötzlich >SuperBürger gegenüber[stehen], denen eine ganze Organisation zur Hand steht, um ihre Vorstellungen vom Gemeinwohl zu verwirklichen.« (Ebd.)

\subsubsection{Transparenzstandards}

")Es ist ja nicht immer klar, wie viel Stiftungen mit Unternehmen zu tun haben, die einen ähnlichen Namen tragen.« (Donsbach/Brade 2013, S. 31) Damit leiten Wolfgang Donsbach und Anne-Marie Brade (2013) eine der Fragen ein, mit der sie die Wahrnehmung forschungsfördernder Stiftungen bei deren Stakeholdern erheben wollen. Doch warum ist das eigentlich >nicht immer klars? Auf die Frage könnte man mit einem gängigen Bonmot über die mangelnden gesetzlichen Transparenzverpflichtungen von Stiftungen antworten:»Jeder Betreiber einer Würstelbude muss mehr offenlegen als eine Stiftung. " ${ }^{33}$ Gemeinnützig anerkannte Stiftungen sind in Deutschland nicht dazu verpflichtet, ihre Satzung zu veröffentlichen, Rechenschaft über ihre Aktivitäten, ihr Stiftungsvermögen, ihr jährliches Stiftungsbudget, ihre Mittelherkunft und -verwendung abzulegen. ${ }^{34}$

Jedoch lässt sich - im Gegensatz zur Würstelbude - ein essenzielles öffentliches Interesse an der Mittelherkunft und -verwendung von Stiftungen begründen, wie es auch gegenüber anderen gemeinwohlorientierten (oder auch öffentlich finanzierten) Institutionen formuliert wird (vgl. Schuler 2010, S. 17). Zum einen sind gemeinnützig anerkannte Stiftungen in öffentlich relevanten Bereichen (wie in der Wissenschaft, im Bildungs-, Sozial- und Kulturbereich) aktiv und beeinflussen damit öffentliche Belange, die alle angehen. Zum anderen sind Stiftungen aufgrund der steuerlichen Vergünstigungen als "öffentlich subventioniert (Adloff 2010, S. 415) zu betrachten. So hebt Frank Adloff hervor, dass ein

33 Dies sei ein Bonmot unter Wirtschaftsprüfer*innen und Steuerberater*innen (vgl. Schuler 2010, S. 218).

34 Schulers wissenschaftsjournalistische Analyse der Bertelsmann Stiftung zeigt beispielhaft die fehlenden Transparenzstandards im Stiftungssektor auf: Es verlangt umfangreiche Recherchearbeiten, wenn man sich für das Stiftungsvermögen, die Herkunft der Stiftungseinahmen und deren Verwendung interessiert (vgl. Schuler 2010). 
»Teil des sonst anfallenden Steueraufkommens der politischen Entscheidung entzogen wird. Stiftungen nehmen also Einfluss auf die öffentliche Ressourcenallokation und setzen eigene Förderschwerpunkte, die völlig abweichend von denen des Staates sein können « (ebd., S. 378). ${ }^{35}$

Dass Stiftungen völlig andere Förderschwerpunkte als der Staat setzen, muss dabei keineswegs problematisch sein bzw. wird oftmals als Chance gesehen. Jedoch könnte für Stiftungen dann trotzdem das gelten, was auch für öffentliche Einrichtungen (wie Bundesbehörden) gilt, nämlich das Informationsfreiheitsgesetz, das Bürger*innen das Recht auf Akteneinsicht gewährt. ${ }^{36}$ Das Gesetz beinhaltet ein verbrieftes Recht für Bürger*innen, Unterlagen einsehen zu dürfen. Bei gemeinnützigen Stiftungen können Bürger*innen das nicht - obwohl sie ja eben gemeinnützig sind und sich daraus m.E. auch ein Anspruch der Öffentlichkeit auf die Transparenz dieser Stiftungen ableitet.

Wie die stiftungsabhängige "private Vision des Gemeinwohls« (Adloff 2010, S. 415) aussieht, kann (zumindest ohne größeren Recherche-Aufwand) momentan nur anhand der Materialien beurteilt werden, die Stiftungen im Rahmen ihrer eigenen Öffentlichkeitsarbeit bereitstellen. Dass dies unzureichend ist, zeigt Schuler an zahlreichen Beispielen in seiner Analyse der Arbeit der Bertelsmann Stiftung. So rechnet die Stiftung beispielsweise ihre Vermögenswerte klein, sodass »die Ausschüttung in der Bilanz der Stiftung nicht ganz so gering erscheint, wie sie eigentlich ist « (Schuler 2010, S. 75).

Ein großes Transparenzdefizit ist dabei, dass es bis heute kein öffentlich gefördertes (und öffentlich zugängliches) Register gibt, das alle Stiftungen, die in Deutschland wirken, erfasst. ${ }^{37}$ Zuletzt wurde ein solches Register in einem Gesetzentwurf aus dem Jahr 1997 vorgeschlagen, seine Notwendigkeit wird also auf politischer Ebene nun schon seit 20 Jahren erkannt (vgl. Deutscher Bundestag 1997). In den USA gibt es ein Stiftungsregister, das über die Angaben des BDS hinausgeht, jedoch ebenfalls käuflich erworben werden muss. In diesem sind die Informationen verarbeitet, die Stiftungen gegenüber der Steuerbehörde machen müssen, darunter auch die Gehälter ranghoher Stiftungsmitarbeiter*innen (vgl. Schnurbein/Wyser/Steffen 2012, S. 14). Obwohl in den letzten Jahren Stiftungen (und damit auch unternehmensnahe Stiftungen) in Deutschland steuerlich stark begünstigt wurden, gibt es immer noch kein Stiftungsregister.

35 Adloff verweist hier auf Münkler (2007, S. 205).

36 Dieser Hinweis ist der Analyse von Schuler (2010, S. 17) oder auch von Holland-Letz (2015, S. 151f.) zu entnehmen.

37 Der Bundesverband Deutscher Stiftungen und das Maecenata Institut haben zwar ein Cesamtregister, jedoch muss man sich den Zugang dazu käuflich erwerben. Auf den jeweiligen Webseiten wird jeweils nur ein Teil der Stiftungen bzw. der Informationen angezeigt. 
Viele Stiftungen unterliegen der Stiftungsaufsicht des jeweiligen Bundeslandes. Diese überprüft jedoch nur, ob die Stiftungen in den angegebenen Stiftungszwecken engagiert sind - sie ist eine rechtliche, keine inhaltliche Aufsicht: "Die Stiftungsaufsicht ist eine reine Rechtsaufsicht. Die Aufsichtsbehörde darf daher nicht an Stelle der Stiftungsorgane Entscheidungen treffen. (Wigand et al. 2015, S. 55) Des Weiteren diene die Aufsicht »dem Interesse des Stifters, nicht dem Interesse des Staates. Sie hat den Zweck, die Stiftung vor Schäden zu bewahren. Die Behörden prüfen, ob der Stifterwille verwirklicht wird und ob die Gesetze eingehalten werden« (ebd.). Von daher werde der Stiftungsaufsicht zwar »eine Kontrollund Überwachungsfunktion dahingehend zugesprochen, die Gemeinwohlverträglichkeit einer Stiftung zu überprüfen« (Bundesverband Deutscher Stiftungen 2016). Doch da sich der Blick der Aufsicht nur auf die Stiftung, nicht aber auch auf die Unternehmen richtet, die mit der Stiftung (auf unterschiedliche Weise) verbunden sind, können Überschneidungen nicht in den Blick geraten (vgl. Füller 2015, S. 7). Etliche große Stiftungen sind zudem als $\mathrm{GmbH}$ organisiert, wie die Stiftung Mercator oder die Robert Bosch Stiftung, und unterliegen gar nicht der Stiftungsaufsicht. Zur letztgenannten merkt Adloff an:

»Bei der Robert Bosch Stiftung hat die Rechtsform dezidiert mit der Ablehnung der Stiftungsaufsicht zu tun. Sie geht auf den Willen Robert Boschs zurück, der keine staatliche Kontrolle wünschte und die Stiftungstätigkeit als Verlängerung der Unternehmensführung betrachtete.« (Adloff 2010, S. 389)

Dass der Anspruch der Öffentlichkeit auf Transparenz auch z.T. im Stiftungswesen anerkannt wird, zeigt sich daran, dass der größte deutsche Stiftungsverband, der Bundesverband Deutscher Stiftungen, die Initiative Transparente Zivilgesellschaft unterstützt. Diese Initiative hat - unterstützt vom BDS und anderen Trägern im Jahr 2010 minimale Transparenzstandards für gemeinnützige Organisationen entwickelt, denen sich Stiftungen freiwillig anschließen können. $\mathrm{Zu}$ den Kriterien gehören u.a. die Veröffentlichung der Satzung, die Berichterstattung über die Tätigkeiten, Angaben zu Vermögen und zur Mittelverwendung und -herkunft (einschließlich Großspenden) sowie zu Namen und Funktionen von Entscheidungsträger*innen (vgl. Transparency International Deutschland 2017a). ${ }^{38}$

Die Entwicklungen im Stiftungswesen, die unter demokratischen Gesichtspunkten problematisch sind, werden - wenn überhaupt - in der Stiftungsfachliteratur oftmals nur kurz benannt, nicht aber kontrovers diskutiert. Stattdessen liegt der Schwerpunkt meist auf der Frage, wie eine bessere und effizientere Stiftungs-

38 Zur Einhaltung dieser Standards durch unternehmensnahe Stiftungen, die auf dem Feld der politischen Bildung aktiv sind: vgl. Kapitel 4.2.1. 
arbeit aussehen könnte. ${ }^{39}$ So wird zwar immer wieder auf die elitäre Ausrichtung von Stiftungen oder auch auf fehlende Stiftungsregister hingewiesen (vgl. European Commission 2015a, S. 119, 9), dennoch wird gleichzeitig gefordert, dass gesetzliche Barrieren für die Gründung von Stiftungen abgebaut und sie noch stärker steuerlich begünstigt werden sollen (vgl. dazu die Forderungen aus der Stiftungsforschung oder von der Bundesregierung: European Commission 2015a, S. 18; Bundesministerium für Arbeit und Soziales 2013, S. XLVIII). Stiftungsregister und Transparenzstandards werden oftmals nicht als Voraussetzung für solche Erleichterungen genannt und erscheinen somit schnell als fakultative Verzierung.

Dabei fällt in Hinblick auf die Stiftungsforschung auf, dass zahlreiche Forschungsarbeiten entweder von Stiftungen beauftragt oder/und von stiftungsnahen Instituten erarbeitet worden sind. So publiziert das Maecanata Institut zwei Schriftenreihen (Maecenata Schriften, Reihe Opuscula), in denen sich etliche Studien zum Stiftungswesen finden. Der Direktor des Instituts ist Rupert Graf Strachwitz, der über 20 Jahre der Geschäftsführer der Maecenata Management $\mathrm{GmbH}$ war, einer Dienstleistungs- und Beratungsgesellschaft für Stiftungen und andere gemeinnützige Organisationen. Der Bundesverband Deutscher Stiftungen gibt ebenfalls regelmäßig erscheinende Publikationsreihen heraus (StiftungsReport, StiftungsRatgeber, Magazin StiftungsWelt). Einschlägige Studien werden vom Centrum für soziale Investitionen und Innovationen (CSI) erarbeitet, das u.a. die Robert Bosch Stiftung, die Fritz Thyssen Stiftung, die Deutsche Bank Stiftung und die Hertie Stiftung finanzieren, so z.B. auch die Studie von Anheier et al. (2017a, b, c). Diese 2017 erschienene, systematische Erforschung des deutschen Stiftungswesens ist ein gemeinsames Projekt des CSI mit der Hertie School of Governance und wurde als

»Kooperationsprojekt [...] von der Fritz Thyssen Stiftung, der Cemeinnützigen Hertie-Stiftung, der Robert Bosch Stiftung, der Stiftung Mercator, der Volkswagen Stiftung und dem Stifterverband für die Deutsche Wissenschaft gefördert.« (Anheier 2016, S. 4)

Die stiftungsfinanzierte Stiftungsforschung verweist somit recht eindringlich auf Probleme mangelnder Distanz der Forschung zum Gegenstand sowie auf eine we-

39 Ein Beispiel dafür ist die Studie von Donsbach/Brade (2013). 2014 hat das Centrum für Soziale Innovationen und Investitionen (CSI) die Studie »Philanthropy and Education - Strategies for impact « veröffentlicht, die den Forschungsbedarf über das Wirken von Stiftungen im Bildungsbereich aufgreift (vgl. Thümler et al. 2014). Die von der Stiftung Mercator finanzierte Studie erforscht die Wirkungsweise und Effizienz von Stiftungen im Bildungsbereich. Im Gegensatz dazu thematisiert die nicht stiftungs-finanzierte Forschung auch die Legitimität und die Zielsetzungen von Stiftungen im Bildungsbereich (vgl. hierzu u.a. Höhne/Schreck 2009; Höhne 2012, 2016), ist aber in der zunehmend unter- und drittmittelfinanzierten Forschungslandschaft nicht stark ausgeprägt. 
nig pluralistische Landschaft der Stiftungsforschung: Philanthropie-kritische (jedoch nicht per se ablehnende) Positionen, die nicht a priori von der Legitimität von Stiftungen ausgehen, sind in der Forschung über Stiftungen (in Deutschland) bislang eher selten zu finden.

\subsection{Unternehmensnahe Stiftungen auf dem Feld der politischen Bildung}

\subsection{1 Überblick über Stiftungen}

Oftmals war jetzt schon die Rede von der Robert Bosch Stiftung oder der Bertelsmann Stiftung, jedoch agieren auch viele andere unternehmensnahe Stiftungen auf dem Feld der politischen Bildung (vgl. dazu auch die Auflistung weiter unten). Damit wird die Dringlichkeit systematischer Untersuchungen dieser Stiftungen und ihrer Aktivitäten deutlich. Diese ist erschwert, z.B. hinsichtlich der Zusammenstellung eines Datensatzes mit unternehmensnahen Stiftungen, die im Bereich der politischen Bildung als wichtige Akteure gelten können. Denn in den vorhandenen Stiftungsdatenbanken ${ }^{40}$ kann nicht gezielt nach unternehmensnahen Stiftungen gesucht werden - wohl auch, weil die Definition(en) zu unternehmensnahen Stiftungen ungeklärt bzw. umstritten sind (vgl. Kapitel 4.1.1). Auch eine Suche nach eindeutig unternehmensnahen Stiftungstypen (wie der Unternehmensstiftung, bei der ein Unternehmen eine Stiftung gründet und die somit über die Identifikation des Stifters klar als sunternehmensnahく bzw. unternehmensverbunden erkennbar wäre) ist momentan nicht möglich.

Auch die Rechtsform einer Stiftung liefert keinen Anhaltspunkt für eine systematische Identifikation unternehmensnaher Stiftungen: Unternehmensverbundene Stiftungen sind beispielsweise als $\mathrm{GmbH}$ (Robert Bosch Stiftung) oder auch als private Stiftung bürgerlichen Rechts (Körber Stiftung) organisiert. Auch gibt es keine Möglichkeit, eine Grundgesamtheit der Stiftungen zusammenzustellen, die in der politischen Bildung tätig sind, um daraus dann im zweiten Schritt unternehmensnahe Stiftungen >zu filtern ‘. Denn in der politischen Bildung tätige Stiftungen geben unterschiedliche Stiftungszwecke an: z.B. den Zweck >Bildungく, etliche auch >Völkerverständigungく oder >demokratisches Staatswesen $<{ }^{41}$

Nachfolgend wird deshalb ein explorativ angelegter Überblick über unternehmensnahe Stiftungen auf dem Feld der politischen Bildung gegeben. Hierfür soll

40 Hier ist besonders auf die (nur im bestimmten Umfang) öffentlich zugänglichen und bundesweiten Datenbanken des Bundesverbands Deutscher Stiftung und des Maecenata Instituts zu verweisen.

41 Diese Stiftungszwecke können damit als wichtiger, aber nicht hinreichender Indikator für die Identifizierung von Stiftungen in der politischen Bildung dienen. 
kurz das dabei zugrunde gelegte Verständnis von Institutionen und politischer Bildung erläutert werden: Institutionen politischer Bildung können analog zu Zeuner anhand der "Definitionen für Institutionen der Erwachsenenbildung« bestimmt werden (Zeuner 2014, S. 138), mithin als »[...] ein Bedingungsgefüge, das so konstruiert ist, dass organisiertes Lernen von Erwachsenen nicht nur einmal, oder hin und wieder, sondern ausdauernd zustande kommen könnte« (Weinberg 1985, S. 90; zit.n.: Zeuner 2014, S. 138f.). Zeuner verweist darauf, dass man verschiedene Einrichtungstypen unterscheiden könne, darunter nicht nur solche, "die ausschließlich politische Bildung anbieten«, sondern auch jene, bei denen dies nur einen Teil der Angebote ausmacht (vgl. Zeuner 2014, S. 140). ${ }^{42}$ Die hier betrachteten unternehmensnahen Stiftungen sind dabei i.d.R. letztgenanntem Einrichtungstyp zuzuordnen.

Bei der Identifikation von unternehmensnahen Stiftungen als politischen Bildungsakteuren wurde zudem ein weites Verständnis von politischer Bildung zugrunde gelegt, das z.B. auch die Förderung von Bürger*innenbeteiligung und -engagement oder ökonomische Bildung einschließt. ${ }^{43}$ Wie auch im »Monitor politische Bildung «, dem Standardwerk zu politischen Bildungsakteuren in Deutschland, wurde somit »kein einheitliches Konzept von politischer Bildung« verwandt, sondern "politische Bildung variabel und flexibel bestimmt, um nicht von vornherein auf ein zu eng gefasstes Begriffskorsett festgelegt zu sein« (Lange 2010, S. 11).

Einige der nachfolgend identifizierten Stiftungen waren im »Monitor politische Bildung « (als >zivilgesellschaftliche Stiftungen $`$ ) zu finden,${ }^{44}$ die anderen wurden eher über das `Schneeballsystem< identifiziert, was bedeutet, dass diese Stiftungen z.B. als Programmpartner der bereits identifizierten unternehmensnahen Stiftungen agieren oder in der Fachliteratur zur politischen Jugendbildung genannt sind (z.B. als Finanziers von Programmen). Es sei ausdrücklich betont, dass der nachfolgende Überblick keine systematische Erhebung darstellt, sondern das Ergebnis erster Recherchen ist, deren Wert nicht in Vollständigkeit besteht, sondern

42 Zeuner nennt hier als Beispiel, dass auch in »Bürgerinitiativen, Nichtregierungsorganisationen, Kulturzentren, Geschichtswerkstätten, Museen usw.«politische Bildung betrieben werde oder auch durch die Wohlfahrtsverbände, die Bundeswehr, dem Bundesfreiwilligendienst; diese Einrichtungen hätten jedoch andere Hauptaufgaben (vgl. Zeuner 2014, S. 142).

43 Formate zur Bürger*innenbeteiligung und zum Bürger*innenengagement sind relevant, da hier oftmals auch informelle politische Lernprozesse stattfinden; die ökonomische Bildung wiederum ist mit politischer Bildung eng verknüpft, da »[s]eit jeher [...] ökonomische Themen und Kompetenzen zum Kern des sozialwissenschaftlichen Lernbereichs [gehören] « (Hedtke 2014, S. 312).

44 Dies sind: Robert Bosch Stiftung, Gemeinnützige Hertie-Stiftung, ZEIT-Stiftung, Körber Stiftung, Freudenberg Stiftung, Bertelsmann Stiftung (vgl. Lange 2010, S. 6). 
darin, dass die Recherchen das Spektrum an (unterschiedlichen) unternehmensnahen Stiftungen und Stiftungsprogrammen explorativ sichten. Damit soll auf den Facettenreichtum eines bisher wenig beachteten Forschungsgegenstands aufmerksam gemacht werden.

Unternehmensnahe Stiftungen

$\mathrm{Zu}$ den identifizierten Unternehmensstiftungen - mithin also Stiftungen, die von Unternehmen gegründet und finanziell ausgestattet werden - gehören:

- BMW Stiftung Herbert Quandt

- DFL Stiftung (bis 2017: Bundesliga Stiftung) ${ }^{45}$

- Deutsche Bahn Stiftung

- innogy Stiftung (bis 2016: RWE Stiftung)

- TUI Stiftung

- Vodafone Stiftung

$\mathrm{Zu}$ den identifizierten unternehmensverbundenen Stiftungen - mithin also Stiftungen, die Anteile an Unternehmen halten - gehören:

- Bertelsmann Stiftung

- Freudenberg Stiftung

- Körber Stiftung

- Robert Bosch Stiftung (RBS)

Was bedeutet diese Aufzählung für die politische Bildung? Ist bei diesen Stiftungen davon auszugehen, dass ihre gemeinnützigen Stiftungsaktivitäten - also auch die in der politischen Bildung - zumindest teilweise an Unternehmensinteressen ausgerichtet werden? Empirische Forschung, die dieser Frage systematisch und vergleichend nachgeht, existiert bislang nicht. Jedoch wird im öffentlich-medialen Diskurs und auf Basis journalistischer Recherchen genau diese Frage diskutiert: am Beispiel der Förderung der Digitalisierung im Bildungsbereich durch die Unternehmensstiftung Deutsche Telekom Stiftung und die unternehmensverbundene Bertelsmann Stiftung. Sowohl für die Deutsche Telekom als auch für den Bertelsmann-Konzern ist das Thema Digitalisierung geschäftspolitisch relevant (vgl. Füller 2015).

Für den Typ der Unternehmens- bzw. CSR-Stiftung (wie eben der Deutschen Telekom Stiftung) ist es nicht unüblich, produktnahe gemeinnützige Programme

45 Die DFL Stiftung ist zwar eine CSR-Stiftung, weist aber einige Besonderheiten auf (vgl. dazu ausführlich Kapitel 4.3.3.4). 
zu fördern: »Die Unternehmensbotschaften sollen mit Hilfe der Errichtung und Tätigkeit einer gemeinnützigen Stiftung besser vermittelt werden können.« (Mecking 2010, S. 382) Damit ist umgekehrt jedoch nicht gesagt, dass eine Unternehmensstiftung immer produktnahe gemeinnützige Programme fördert: Es kann hinsichtlich des Stiftungszwecks entschieden werden, »[...] ob die Stiftung in einem Bereich tätig ist, der in inhaltlicher Nähe zum Unternehmensgegenstand liegt oder ob die Stiftung [...] inhaltlich in unternehmensfremden Bereichen tätig ist (ebd., S. 379).

Die (oftmals wirtschaftswissenschaftliche) CSR-Fachliteratur hebt das »Potential« von Corporate-Citizenship-Maßnahmen, wie sie z.B. in Form von CSRStiftungen realisiert werden, hervor: Es läge in seinem sogenannten »Win-winCharakter«, mithin »dem wechselseitigen Vorteil von Unternehmen, gemeinnützig tätigen Partnerorganisationen und gesellschaftlichem Umfeld« (ebd., S. 372). Gleichzeitig sei jedoch die

»[d]ie altruistische Zwecksetzung [...] mit der Erwartung verbunden, ein gesundes gesellschaftliches Umfeld für die eigene (erwerbs-)wirtschaftliche Ceschäftstätigkeit zu schaffen sowie innerhalb der Zielgruppe das Ansehen des Unternehmens zu steigern und den Umsatz und den Ertrag zu optimieren.« (Ebd., S. 371f.) ${ }^{46}$

CSR ist somit im Rahmen von "strategische[r] Unternehmenskommunikation « (Biedermann 2008, S. 291-306) und teilweise auch in Verbindung mit Lobbyismus (vgl. Speth 2010) zu betrachten. Ulrich Brömmling konstatiert, dass »[e]in schmaler Grat« zwischen »Unternehmenszielen und stifterischen Aktivitäten der zugehörigen Unternehmensstiftungen« läge (Brömmling 2011, S. 15). Der Geschäftsführer der Deutschen Telekom Stiftung, Ekkehard Winter, befindet, dass man »[n]atürlich nicht so nah am Kerngeschäft « sein sollte, »dass die Gemeinnützigkeit gefährdet ist «, gleichzeitig »[a]ber bezüglich der bearbeiteten Themen auch nicht so weit weg, dass man keinen Zusammenhang zwischen Stifterunternehmen und Unternehmensstiftung mehr erkennen kann« (Winter; zit.n.: Brömmling 2011, S. 15).

Bei unternehmensverbundenen Stiftungen, die Anteile an Unternehmen halten, ${ }^{47}$ wird oftmals - ganz im Gegenteil zu den Unternehmensstiftungen - hervorgehoben, das philanthropische und geschäftliche Felder strikt getrennt seien (vgl.

46 Politikwissenschaftliche Forschung, die z.B. den Fokus auf damit umgesetzte, unternehmerische Partikularinteressen im (deutschen) Bildungsbereich setzt, gibt es bisher nicht.

47 Wobei an dieser Stelle nur die >Unternehmensträgerstiftungen im weiten Sinn< gemeint sind und ausdrücklich nicht die >Unternehmensträgerstiftungen im engen Sinn<, mithin Unternehmen, »die in der Rechtsform der Stiftung geführt werden « (Eulerich 2016, S. 110). Vgl. zu diesen Unterscheidungen die Ausführungen zu unternehmensverbundenen Stiftungen in Kapitel 4.1.1. 
die Ausführungen zur Robert Bosch Stiftung in Kapitel 4.3.1). Das Beispiel Bertelsmann Stiftung zeigt jedoch, dass auch unternehmensverbundene Stiftungen nicht generell >unternehmensfern im Rahmen ihrer gemeinnützigen Aktivitäten agieren. Interessant ist, dass sich in beiden Stiftungen, die zu unterschiedlichen unternehmensnahen Stiftungstypen gehören, höchste Konzernvertreter*innen im Stiftungsvorstand befinden. ${ }^{48}$ Bei der Bertelsmann Stiftung wird die (z.T. vorgefundene) Ausrichtung von Stiftungsaktivitäten im Unternehmensinteresse in Verbindung mit diesen Personalunionen diskutiert (vgl. Füller 2015): Dadurch besteht die Möglichkeit, dass die Stiftung im Unternehmensinteresse genutzt wird. Personalunionen zwischen höchsten Stiftungs- und Unternehmensgremien können dabei als gängige Praxis gelten und finden sich in vielen der hier genannten Stiftungen, z.B. auch bei der RBS oder der Freudenberg Stiftung (vgl. Kapitel 4.3.1). ${ }^{49}$ Sie sind nur oftmals nicht so leicht ersichtlich, wie am Beispiel der RBS noch ausführlich erläutert werden soll (vgl. ebd.).

Unternehmensstiftungen wird dabei offen zuerkannt, dass es »[...] durchaus üblich [ist], dass Führungskräfte des Unternehmens auch im Entscheidungsgremium der Stiftung vertreten sind und dort die Richtlinien der Stiftungspolitik mitbestimmen« (Habighorst/Seltenreich 2007). Auf diese Weise lasse sich »eine Koordinierung der Interessen [...] über die Besetzung der Stiftungsgremien einrichten«, obwohl »die unternehmensnahe Stiftung und das Unternehmen [...] rechtlich selbstständig und unabhängig nebeneinander [stehen] (ebd.). Was dies für die politische Bildungsarbeit bedeutet, wird nachfolgend noch ausführlicher dargestellt am Beispiel der DFL Stiftung, die Partner der RBS im Programm »Lernort Stadion« ist (vgl. Kapitel 4.3.3.4), sowie in der Fallstudie beleuchtet (vgl. Kapitel 5.2).

\section{Weitere Stiftungen}

Wie in Kapitel 4.1.1 erläutert, kommt es bei der Definition sunternehmensnaher Stiftungen darauf an, welche Dimensionen (z.B. rechtliche, finanzielle, personelle

48 Liz Mohn und ihre Tochter Brigitte Mohn sind nicht nur im Vorstand der Stiftung (vgl. Bertelsmann Stiftung 2017), sondern gleichzeitig auch Vorsitzende (Liz Mohn) und Cesellschafterin (Brigitte Mohn) der Bertelsmann Verwaltungsgesellschaft mbH (BVC) (vgl. Bertelsmann 2017a). Diese »kontrolliert die Stimmrechte in der Hauptversammlung der Bertelsmann SE \& Co. KGaA [...]. Darüber hinaus kontrolliert die BVG die Stimmrechte in der Hauptversammlung der Bertelsmann Management SE.«(Bertelsmann 2017b) Dies sind nur die einschlägigsten Personalunionen; es gibt weitere zwischen dem Kuratorium der Stiftung auf der einen Seite und der BVG oder dem Aufsichtsrat des Unternehmens auf der anderen Seite. Bei der Deutschen Telekom Stiftung ist Thomas Dannenfeldt nicht nur Vorstands- und Kuratoriumsmitglied der Stiftung, sondern auch »Vorstandsmitglied Finanzen Deutsche Telekom AG « (Deutsche Telekom Stiftung 2017). Dies ist nur die einschlägigste Personalunion, es gibt weitere zwischen Kuratorium und höchsten Unternehmenspositionen.

49 Beide Stiftungen (RBS und Freudenberg Stiftung) haben zudem nur ein Stiftungsorgan - ein Kontrollorgan fehlt. 
Verbindungen) zugrunde gelegt werden. Nachfolgend sollen einige Stiftungen aufgeführt werden, die nicht zu den Unternehmensstiftungen oder unternehmensverbundenen Stiftungen gezählt werden, über deren >Unternehmensnäheく aber dennoch diskutiert werden kann. Sie wurden von Unternehmer*innen gegründet ${ }^{50}$ oder/und haben viele Personen aus dem Wirtschaftsleben in ihren Gremien sitzen oder/und finanzieren sich in beträchtlichem Maße durch Mittel von Unternehmen und unternehmensnahen Stiftungen. ${ }^{51}$

- Gemeinnützige Hertie-Stiftung: Sie wurde 1974 gegründet und gehört mit 1 Mrd. Stiftungsvermögen zu den größten privaten Stiftungen in Deutschland. Ihre Stifter sind Brigitte Gräfin von Norman und Hans-Georg Karg, der Inhaber des Hertie Waren- und Kaufhauses war (vgl. Gemeinnützige Hertie Stiftung 2017a). Während ursprünglich »97,5 Prozent der Anteile der Hertie Waren- und Kaufhaus $\mathrm{GmbH}$ « in die Stiftung eingebracht wurden, habe sich die Stiftung $1998 »[\mathrm{~m}]$ it dem Verkauf ihrer Karstadt-Anteile an Quelle aus ihrer Unternehmensbindung « gelöst (ebd.). ${ }^{52}$ Die Stiftung war in diverse Steuer-Skandale verwickelt. ${ }^{53}$ Im Vorstand und Kuratorium lassen sich viele (z.T. auch ehemalige) Repräsentant*innen aus der Wirtschaft finden. ${ }^{54}$

50 Während es in der US-amerikanischen Fachliteratur üblich ist, von Unternehmer*innen bzw. Unternehmensfamilien gegründete Stiftungen als >corporate foundations zu bezeichnen, wird in Deutschland oft von sprivaten « Stiftungen gesprochen. Von einigen werden sie dennoch als sunternehmensnah< geführt: vgl. Junck 2014, S. 12-14; Brömmling 2011, S. 11; Striebing 2017b, S. 84.

51 Es gibt weiterhin Stiftungen, die von Arbeitgeber-, Arbeitnehmer- und Unternehmensverbänden gegründet wurden; diese konnten hier nicht berücksichtigt werden.

52 In der Fachliteratur wird die Gemeinnützige Hertie Stiftung z.T. noch als unternehmensnahe Stiftung geführt (vgl. Hardtke/Kleinfeld 2010, S. 63).

53 Robert Jacobi schreibt in einem Artikel in der Welt, dass »die Gemeinnützige Hertie-Stiftung [...] in den neunziger Jahren als finanzieller Verschiebebahnhof [diente], um die Übernahme der Kaufhauskette durch Karstadt steuersparend gestalten zu können.«(Jacobi 2009)

54 Im Vorstand sitzen u.a. Bernd Knobloch (ehemaliges Vorstandsmitglied der Commerzbank AG, ehemaliger Vorstandsvorsitzender der Eurohypo AG), Rainer Neske (Vorsitzender des Vorstands Baden-Württembergische Bank und der Landesbank Baden-Württemberg) und Gordon Riske (Vorsitzender des Vorstands der KION GROUP AG). Im Kuratorium sitzen u.a. Michael Endres (ehemaliges Vorstandsmitglied der Deutsche Bank AG), Hans-Jörg Vetter (ehemaliger Vorsitzender des Vorstands Baden-Württembergische Bank und der Landesbank Baden-Württemberg), Andreas Barner (ehemaliger Vorstandsvorsitzender Boehringer Ingelheim Pharma CmbH \& Co. KG; jetzt: Mitglied des Gesellschaftsausschusses der C.H. Boehringer Sohn AC \& Co. KG), Nico Hofmann (Co-CEO/Ceschäftsführer UFA CmbH, Regisseur, Drehbuchautor), Frank Mattern (Senior Partner Deutschland McKinsey \& Company Inc.), Nikolaus von Bomhard (ehemaliger Vorsitzender des Vorstands Münchener RückversicherungsCesellschaft) (vgl. Gemeinnützige Hertie Stiftung 2017b). 
- Heinz NixdorfStiftung: Sie ist »[a]us dem Nachlass des 1986 verstorbenen Unternehmers Heinz Nixdorf " gemeinsam mit der Stiftung Westfalen hervorgegangen (Heinz Nixdorf Stiftung 2017c).

- Herbert Quandt Stiftung: Sie wurde 1980 als Unternehmensstiftung von dem Chemiekonzern Atlana AG gegründet, »2007 erhielt die Stiftung im Zuge von strukturellen Veränderungen der ALTANA AG eine Zustiftung über 15 Mio. Euro von der Unternehmerin und Stiftungsratsvorsitzenden Susanne Klatten « (Herbert Quandt Stiftung 2017c); Klatten ist "milliardenschwere Erbin der BMWEigentümerfamilie Quandt« (Der Spiegel 2015). 2016 hat die Stiftung ihre operative Arbeit eingestellt (vgl. Herbert Quandt Stiftung 2017c), war aber jahrelang auf dem Feld der politischen Bildung tätig. ${ }^{55}$

- Joachim Herz Stiftung: Das Vermögen der Joachim-Herz-Stiftung stammt aus dem Privatvermögen des Unternehmers Joachim Herz, der 2008 verstorben ist; die Stiftung wurde im selben Jahr von seiner Frau Petra Herz gegründet (vgl. Joachim Herz Stiftung 2017d). Das Vermögen setzt sich zu rund 75 Prozent aus »unternehmerische[n] Beteiligungen« zusammen, wobei »[d]er Schwerpunkt [...] in der Beiersdorf AG« liegt (dies. 2017c).

- Roland Berger Stiftung: Sie wurde 2008 von Roland Berger gegründet, der »Ehrenvorsitzender des Aufsichtsrats der Münchner Strategieberatung Roland Berger $\mathrm{GmbH}$ « ist (Roland Berger Stiftung 2017a). Die von ihm gegründete Unternehmensberatung Roland Berger $\mathrm{GmbH}$ gehört $\mathrm{zu}$ den »weltweit führenden Strategieberatungen und berät internationale Industrie- und Dienstleistungsunternehmen sowie öffentliche Institutionen« (ebd.). Roland Berger ist Mitglied des Kuratoriums der Stiftung, das "satzungsgemäß in allen grundsätzlichen Angelegenheiten der Stiftung [entscheidet]« (dies. 2017b). Gleichzeitig ist er »als Investor tätig [...] in zahlreichen Unternehmen und Start-Ups« sowie »Mitglied verschiedener Aufsichts- und Beiräte von nationalen und internationalen Unternehmen, Stiftungen und Organisationen;

55 Beispielhaft für ihre Aktivitäten sei hier genannt, dass die Herbert-Quandt-Stiftung u.a. den Programmschwerpunkt »Trialog der Kulturen«hatte, in dessen Rahmen sie »[j]edes Schuljahr [...] Lehrer und Schüler aller Schultypen und ]ahrgänge ab Stufe fünf ein[lud], eigenständige und kreative Beiträge zur Wissensvermittlung über die drei Kulturen zu entwickeln und Wege zu einer vertieften Reflexion über das Verhältnis von Religion und Staat zu finden«(Herbert Quandt Stiftung 2017a). Auch führte sie das Programm »Bürger.Innen.Land - Für eine aktive Zivilgesellschaft in Mecklenburg-Vorpommern«durch, das u.a. auf die Stärkung von »Demokratiebewusstsein«abzielte: »Angesichts vielfältiger Herausforderungen in Mecklenburg-Vorpommern entsteht bei den Menschen häufig das Gefühl, ihr Cemeinwesen könne die anstehenden Probleme nicht mehr lösen. Von diesem Frustrationspotenzial profitieren verstärkt extremistische Parteien und Gruppierungen. Bürgerschaftliches Engagement dagegen stellt den eigenen Ort positiv dar und zeigt, dass die Zivilgesellschaft funktioniert. Eine Stärkung des Bewusstseins für demokratisches Zusammenleben verbessert die Bürgergesellschaft nachhaltig.« (Herbert Quandt Stiftung 2017b) 
derzeit bei der Schuler AG, Göppingen, Rocket Internet SE, Berlin, Banzai S.p.A. Mailand, Geox S.p.A., Biadene di Montebelluna und China Mensheng Investment, Shanghai« (dies. 2017a).

- Stiftung Mercator: Sie wurde 1996 von der »Stifterfamilie Schmidt gegründet, die »neben Haniel und Beisheim zu den Hauptanteilseignern der Metro Group [gehört]« (Stiftung Mercator 2017).

- ZEIT-Stiftung Ebelin und Gerd Bucerius (kurz: ZEIT-Stiftung): Sie wurde 1971 von Gerd Bucerius, dem Gründer der Wochenzeitung DIE ZEIT, ins Leben gerufen; seine Frau Ebelin Bucerius war als Geschäftsführerin im ZEIT-Verlag tätig (vgl. ZEIT-Stiftung 2017).

Die nachfolgenden Stiftungen wurden nicht von Unternehmer*innen oder Unternehmen gegründet. Legt man Gremienbesetzung oder Mittelherkunft als Kriterien zugrunde, weisen diese Stiftungen m.E. dennoch eine gewisse Unternehmensnähe auf:

- Deutsche Kinder- und Jugendstiftung: Sie wurde "auf Initiative der International Youth Foundation und der damaligen Bundestagspräsidentin Rita Süssmuth « gegründet (Wikipedia 2017b). ${ }^{56}$ Ihre Programme werden "größtenteils über Zuwendungen, Spenden oder im Rahmen von Kooperationen finanziert. Dabei stehen die öffentlichen und privaten Gelder etwa im Verhältnis 58 $\mathrm{zu} 42$ (Deutsche Kinder- und Jugendstiftung 2017c). Als $\mathrm{GmbH}$ verfügt die Deutsche Kinder- und Jugendstiftung neben der Geschäftsführung und einem Stiftungsrat über »Gesellschafter«, die »verantwortlich [sind] für die unternehmerischen und strategischen Entscheidungen, für die finanzielle Stabilität und Effektivität« (dies. 2017a). Als Gesellschafter werden neben Einzelpersonen (wie Werner Gegenbauer, dem Aufsichtsratsvorsitzenden der Unternehmensgruppe Gegenbauer) vier unternehmensverbundene Stiftungen aufgeführt: die Freudenberg Stiftung, die Jacobs Foundation, ${ }^{57}$ die Körber-Stiftung und die Robert Bosch Stiftung (vgl. ebd.). Darüber hinaus werden als »Programmpartner und Förderer zahlreiche Unternehmen und unternehmensnahe Stiftungen auf der Website der Deutschen Kinder- und Jugendstiftung genannt (dies. 2017b).

- Landesstiftung Baden-Württemberg: Sie wurde 2000 in der Rechtsform der GmbH gegründet und ihr Stiftungsvermögen von 2,2 Mrd. stammt »im Wesentlichen

56 Es wird hier Wikipedia zitiert, da sich keine sonstigen öffentlich zugänglichen Quellen zur Ceschichte der Deutschen Kinder-und Jugendstiftung finden lassen.

57 Die Jacobs Foundation ist eine Stiftung schweizerischen Rechts, z.T. agiert sie auch im deutschen Bildungsbereich, wie an der Funktion der Gesellschafterin der Deutschen Kinder- und Jugendstiftung deutlich wird. 
aus Investmentfonds, Immobilien und Unternehmensbeteiligungen« (BadenWürttemberg Stiftung 2017).

- Schwarzkopf-Stiftung Junges Europa: Die Stiftung wurde 1971 von Pauline Schwarzkopf gegründet (vgl. Schwarzkopf-Stiftung Junges Europa 2017b), der Frau des Haarkosmetik-Unternehmers Heinz Schwarzkopf. Im Stiftungsvorstand sitzen u.a. Michael Maßbaum (Deloitte), Eric Schweitzer (Präsident des Deutschen Industrie- und Handelskammertags) und Georg Fahrenschon (Präsident des Deutschen Sparkassen- und Giroverbands) (vgl. dies. 2017c).

Dass alle diese Stiftungen als `gemeinnützigく anerkannt sind, sollte Wissenschaft und Öffentlichkeit nicht davon abhalten, genauer hinzuschauen: Welche politischen Positionen und welche Weltanschauungen werden in den Stiftungsprojekten vorangetrieben? Die von den Stiftungen postulierte `Unabhängigkeit und der betont >zivilgesellschaftliche Charakter< von Stiftungen darf nicht dazu führen, diesen Institutionen per se eine von Wirtschaftsinteressen unabhängige Ausrichtung $\mathrm{zu}$ bescheinigen oder umgekehrt eine ggf. vorhandene Wirtschaftsnähe prinzipiell als eine zu interpretieren, die im Interesse aller Gesellschaftsmitglieder ist.

Bei diesen Stiftungen ist m.E. grundsätzlich danach zu fragen, inwiefern sie - z.B. aufgrund eines*einer Stifters"in mit Unternehmensvergangenheit - Eigentümer"innen-Interessen (die eben nicht automatisch mit Gemeinwohlinteressen gleichzusetzen sind) nahe stehen. Oder ob diese Stiftungen eine weltanschauliche Nähe zu unternehmerischen Prinzipien (wie Wettbewerb, Eigentumsrechten und Eigenverantwortung) haben. Unabhängig davon, ob man hier der These der sneoliberalen Ausrichtung، folgen mag oder nicht, handelt es sich bei den Stiftungen um Institutionen der gesellschaftlichen Eliten, denen nicht pro forma unterstellt werden kann, Bildungsförderung im Sinne der Allgemeinheit zu betreiben. Ob eine - wie auch immer geartete - Unternehmensnähe (z.B. durch Personalunionen) Einfluss auf die Ausrichtung der konkreten Bildungsarbeit hat, die oftmals mit Partnern aus dem öffentlichen und zivilgesellschaftlichen Bereich umgesetzt wird, kann mangels empirischer Forschung nicht beurteilt werden. Dieser Frage wird deshalb noch in einer Fallstudie nachgegangen (vgl. Kapitel 5).

\section{Transparenz}

Die Identifikation von Personalunionen in höchsten Stiftungsgremien - als erster Hinweis für eine mögliche Ausrichtung von Stiftungstätigkeiten im Unternehmensinteresse - ist mit einem unerwartet hohen Rechercheaufwand verbunden. Dies liegt u.a. daran, dass der Großteil der Stiftungen seine Satzungen nicht veröffentlicht und so die genaue Funktion der Organe, ggf. sogar die Anzahl, oftmals nicht bekannt ist. Um überhaupt eine Unternehmensnähe zu klären, müsste zudem die Vermögenszusammensetzung veröffentlicht werden, da dadurch ersicht- 
lich würde, ob Unternehmensbeteiligungen, Aktien etc. zum Vermögen gehören. Dadurch würde es möglich, im zweiten Schritt zu analysieren, mit welchen Unternehmen es relevante Personalunionen geben könnte.

Kurzum: Gesetzlich verpflichtende Transparenzstandards für Stiftungen wären wichtig, jedoch gibt es diese nicht. Es existieren generell keine »(e)inheitliche[n] Veröffentlichungspflichten für gemeinnützige Organisationen [...] in Deutschland« (Transparency International Deutschland 2017c). Es gibt seit 2010 nur die bereits erwähnten freiwilligen Standards der Initiative Transparente Zivilgesellschaft (ITZ). Die Initiatoren - darunter auch der Bundesverband Deutscher Stiftungen - treten dafür ein, dass eine Einrichtung, die "für das Gemeinwohl tätig wird, [...] der Gemeinschaft [...] sagen« sollte, »welche Ziele die Organisation genau anstrebt, woher die Mittel stammen, wie sie verwendet werden und wer die Entscheidungsträger sind« (ebd.) (vgl. auch Kapitel 4.1.3).

Anfang September 2017 haben 912 Organisationen die Initiative unterschrieben (vgl. dies. 2017b), dies ist lediglich ein Bruchteil der gemeinnützigen Organisationen in Deutschland..$^{58}$ Die Beachtung der ITZ-Kriterien kann jedoch gerade bei unternehmensnahen Stiftungen für die Beurteilung ihres gemeinwohlorientierten Charakters als besonders wichtig erachtet werden, da partikulare und gemeinwohlorientierte Interessen eng beieinander liegen können, diese oftmals finanziell gut ausgestattet sind und dementsprechend auch einflussreich sein können (vgl. dazu Kapitel 4.1.1 und 4.1.2). Von den hier zehn genannten (eindeutig zu klassifizierenden) unternehmensnahen Stiftungen nimmt nur die Körber Stiftung an der ITZ teil (vgl. Tabelle 2). Tabelle 2 zeigt weiterhin, dass nur eine der zehn Stiftungen eine Satzung und nur vier von zehn Stiftungen einen regelmäßigen Jahresbericht veröffentlichen. Dabei wären Satzung und Jahresbericht sehr wichtig, um einen Einblick in Stiftungsstrukturen und -tätigkeiten zu erhalten. ${ }^{59}$

Die Relevanz von gesetzlich verpflichtenden Transparenzstandards kann abschließend am Beispiel der DFL Stiftung (die bis 2017 unter dem Namen Bundesliga Stiftung firmierte) veranschaulicht werden, die aufgrund ihres Programms Lernort Stadion, ${ }^{60}$ das sich an $>$ benachteiligte Jugendliche< richtet, noch näher betrachtet wird (vgl. Kapitel 4.3.3.4). Die Stiftung betont zwar (wie so viele Stiftungen), dass »[i]hre Stiftungsarbeit [...] von [...] größtmöglicher Transparenz [...] geprägt« sei

58 Der aktuelle ZIVIZ-Survey geht von rund 633.922 gemeinnützigen Organisationen aus; der Survey ist eine »repräsentative Befragung zivilgesellschaftlicher Organisationen in Deutschland « und versteht unter »organisierter Zivilgesellschaft $<$ [...] gemeinnützige Organisationen « wie»Vereine und Stiftungen, Cenossenschaften und Kapitalgesellschaften [...]«(Priemer/Krimmer/Labigne 2017, S. 5).

59 Für die Jahresberichte gibt es jedoch keine gesetzlich festgelegten Standards, sodass sich diese im Umfang und Inhalt stark unterscheiden können. Zudem stellen Jahresberichte ein Instrument zur Öffentlichkeitsarbeit dar.

60 Das Programm wird von weiteren Partnern gefördert, vgl. ausführlich dazu Kapitel 4.3.3.3. 
(Bundesliga Stiftung 2012, S. 9), veröffentlicht aber keine Satzung, keinen regelmäßigen Jahresbericht und ist keine Unterzeichnerin der ITZ.

Recherchen zur DFL Stiftung ergaben, dass eine CSR-Vollversammlung existiert, bei der aller zwei Jahre die Mitarbeiter*innen der DFL Stiftung sowie der CSR-Stiftungen und -Abteilungen der Fußballclubs zusammenkommen (vgl. Cologne Business School 2015). ${ }^{61}$ Bei den CSR-Vollversammlungen wird sich mit »wichtigen gesellschaftlichen Themen« befasst, wie auf der Seite von Bayer 04 Leverkusen zu lesen ist - 2015 z.B. mit dem Thema "Flucht und Integration« (Bayer O4 Leverkusen 2015). Im Wechsel mit der CSR-Vollversammlung findet alle zwei Jahre zudem ein »Methodentag der Bundesliga-Stiftung zum sozialen und gesellschaftlichen Engagement des Profifußballs«statt (DFL Stiftung 2017a). 2016 trafen sich »Verantwortliche aus dem Bereich Corporate Social Responsibility (CSR) von 30 Proficlubs« (ebd.). Zum Schwerpunkt der Tagung gehörte u.a. die »Verknüpfung von gesellschaftlichem Engagement und wirtschaftlichem Nutzen«, in deren Rahmen auch »das wirtschaftliche Potenzial des gesellschaftlichen Engagements« diskutiert wurde (ebd.). Da CSR-Stiftungen nicht dem Informationsfreiheitsgesetz unterliegen, sind Unterlagen dieser Gremien (Tagungsberichte/-protokolle, Beschlüsse etc.) nicht öffentlich einsehbar, obwohl mit dem >sozialen Engagement ganz offensichtlich öffentliche Belange berührt sind. Ein Recht auf Einsicht in Stiftungsunterlagen wäre folglich sinnvoll - nicht nur, um Einblick in die Strategieentwicklung zu bekommen, sondern auch, um sich über die Arbeit von CSR-Gremien informieren zu können.

61 Im Februar 2015 wurde ein Gespräch mit einer Mitarbeiterin der DFL Stiftung in Frankfurt a.M. geführt, in dem diese u.a. die CSR-Versammlungen erwähnte. Da es keine öffentlich einsehbaren Dokumente gibt, wurden Informationen zu den CSR-Versammlungen im Internet recherchiert, weshalb hier u.a. die Websites der >Cologne Business Schook und nachfolgend auch die von Bayer o4 Leverkusen zitiert sind. 
Tabelle 2: Erfüllung von Transparenzstandards unternehmensnaher Stiftungen in der politischen Bildung (Stand: 3. August 2017), Quelle: Eigene Recherche

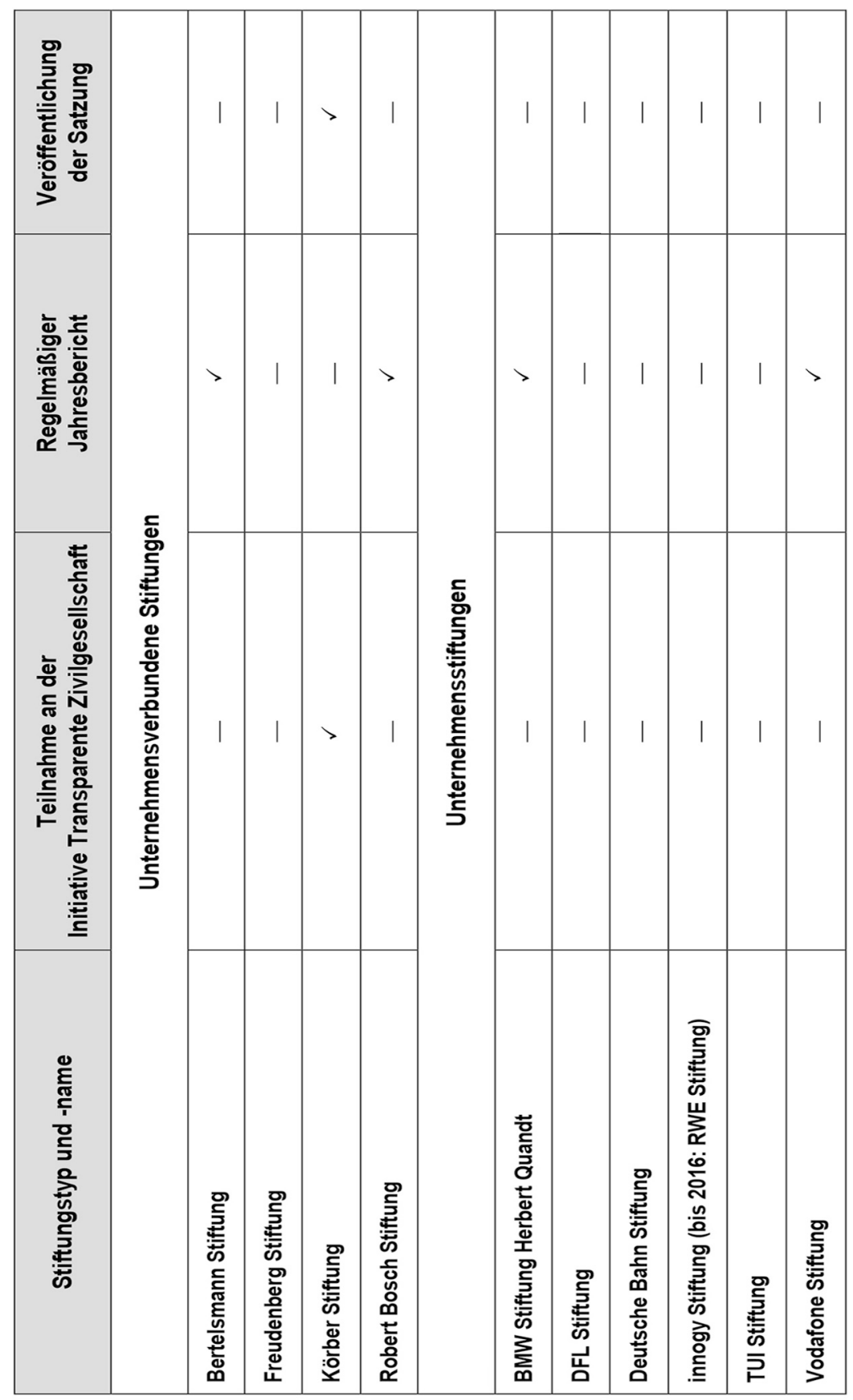




\subsection{2 Überblick über Aktivitäten}

Der Überblick über unternehmensnahe Stiftungen (vgl. Kapitel 4.2.1) deutet bereits darauf hin, dass die »bildungspolitischen Aktivitäten von Stiftungen « eben nicht nur insgesamt »sichtbar zugenommen « haben (Höhne 2016, S. 35), sondern auch in der politischen Bildung. Unter Verweis auf eine feld- und machttheoretische Verortung des Engagements von Stiftungen im Bildungsbereich betont der Erziehungswissenschaftler Höhne, dass »Stiftungen sowohl Ergebnis als auch Katalysatoren einer Privatisierungspolitik sind, die gerade durch ein netzwerkförmig-korporatistisches Politikarrangement staatlicherseits vorangetrieben wird« (ebd., S. 37). Neben der stiftungsfreundlichen Gesetzgebung der letzten Jahre (vgl. Wigand et al. 2015, S. 2f.) und des damit einhergehenden Stiftungsbooms kann dabei die verstärkte Sichtbarkeit unternehmensnaher Stiftungen in der politischen Bildung auch auf die Politik der Engagementförderung der Bundesregierung zurückgeführt werden. So wurde z.B. 2007 die »Förderung bürgerschaftlichen Engagements als gemeinnützig eingestuft und damit als steuerlicher Gemeinnützigkeitszweck anerkannt« (Bundesverband Deutscher Stiftungen 2012b, S. 71). Im Jahr 2010 verabschiedete die Bundesregierung zudem die Nationale Engagementstrategie, mit welcher die Rahmenbedingungen auch für Unternehmen und unternehmensnahe Stiftungen verbessert wurden, um sich verstärkt im Bereich Bürger*innenengagement zu betätigen:

»Die Bundesregierung setzt mit der Engagementstrategie einen Rahmen, der das bürgerschaftliche Engagement der Wirtschaft, der Stiftungen und der Bürgerstiftungen nicht nur anerkennt, sondern auch systematisch unterstützen wird.« (Bundesregierung 2010, S. 4)

Die verstärkten Aktivitäten von unternehmensnahen Stiftungen in der politischen Bildung sind insgesamt vor dem Hintergrund einer zunehmenden Privatisierung öffentlicher Bereiche einzuordnen, die »sich nach der Deregulierung und Privatisierung in Arbeits- und Sozialpolitik nun auch für den Bildungsbereich $a b[z e i c h-$ net] [...]« (Höhne 2016, S. 35). Im Zuge der Privatisierung von Bildung stößt man nicht nur verstärkt auf unternehmensnahe Stiftungen, sondern oftmals auch auf Unternehmen (vgl. Kapitel 5), die sich im Rahmen von Public-private-Partnerships (PPPs), also Öffentlich-privaten Partnerschaften (ÖPP), engagieren. PPPs stellen längst »eine verbreitete Organisationsform der Finanzierung und Erbringung öffentlicher Aufgaben« dar (Gericke 2013) und gelten mittlerweile auch im Bildungsbereich »als aussichtsreiches Gestaltungsmittel« (ebd.). ${ }^{62}$ Fraglich an PPPs allge-

62 Christina Gericke findet den Begriff der Partnerschaft dabei jedoch »irreführend«, da es nicht um eine gleichberechtigte Partnerschaft ginge, sondern PPPs »vielmehr ein wichtiges politisches Instrument zur (Teil-)Privatisierung staatlicher Aufgaben« seien (Gericke 2013). 
mein und so auch an denen im Bildungsbereich ist, ob durch sie die »Qualität der >Dienstleistungen'« erhalten bleibt (ebd.). ${ }^{63}$

Zweifel an unternehmerischen Initiativen in der Beteiligungsförderung, in dessen Kontext es auch zum politischen Lernen kommt oder kommen kann, scheinen angebracht, wenn man die Ausführungen des Journalisten Thomas Wagner zugrunde legt. In seinem Buch »Die Mitmachfalle. Bürgerbeteiligung als Herrschaftsinstrument « von 2013 macht er auf Beteiligungsprojekte aufmerksam, die von Konzernen gefördert wurden und dementsprechend auch Konzerninteressen dienen sollten (vgl. Wagner 2013) ${ }^{64}$ Er zeigt die "politischen Aspekte einer [...] neuen Beteiligungskultur « auf, »in der wichtige Fragen des Zusammenlebens zunehmend in öffentlichen Foren debattiert werden, die von Sponsoren aus der Wirtschaft oder konzernnahen Stiftungen finanziert werden.«(Ebd., S. 14)

Diese Beteiligungskultur deutet er vor dem zeithistorischen Hintergrund: Aufgrund der Forderungen von Bürger*innen nach mehr Beteiligung und dem Verlust »an Bindekraft« von Großorganisationen wie »Parteien, Gewerkschaften und

63 Gericke führt hierzu aus: »Was an ÖPP im Bereich des Gebäudebaus und der Bewirtschaftung kritisiert wird - mangelnde Transparenz, fehlende Kontrollmöglichkeit bei zunehmender Abhängigkeit von den privaten Partnern, schließlich Qualitätsmängel - erfährt auf der Ebene von Schule und Unterricht eine brisante Zuspitzung: Öffentlich-private Partnerschaften werden derzeit weder systematisch erfasst noch inhaltlich geprüft, geschweige denn auf ihre Effekte hin analysiert. (Gericke 2013)

64 Als Beispiel ist der »Fall der Berliner Schneemann-Demo« aus dem Jahr 2010 zu nennen (vgl. Wagner 2013, S. 8): Über soziale Netzwerke waren Menschen zum Protest gegen den Klimawandel aufgerufen. Der hessische Energiekonzern Entega hatte Kunstschnee und Deko-Material bereitgestellt. Durch die Demonstration sollten die Teilnehmenden dazu bewogen werden, »zu dem in der Eigendarstellung ökologischen Stromanbieter Entega zu wechseln. Somit handelte es sich um eine Werbeveranstaltung mit dem Ziel der Neuerschließung des Berliner (Strom)Markts und war entsprechend auch nicht als Demonstration angemeldet worden [...]. Die im Internet angegebenen Kosten der Aktion beliefen sich auf ca. 500.000 Euro «(Hinterhuber/Möller 2013, S. 214; zit.n.: Wagner 2013, S. 8). Wagner benennt zudem das vom Autokonzern BMW finanzierte BMW Guggenheim Lab, in dessen Rahmen »in neun Metropolen über die Probleme von sogenannten Mega-Cities diskutiert werden« sollte (Wagner 2013, S. 21). Die Beschreibungen rund um das Projekt klangen »demokratisch, transparent und an drängenden Problemen der Stadtbewohner orientiert« (ebd., S. 22; die Beschreibungen gibt Wagner ausführlich wieder: vgl. ebd., S. 21f.). Tatsächlich wollte BMW (laut dem damaligen Marketingchef) aber vor allem diejenigen »ansprechen, die heute vielleicht noch keine besondere Affinität zur Marke BMW haben - möglicherweise dem Auto sogar ambivalent gegenüber stehen«; das Projekt diene der»langfristige[n], positive[n] Wahrnehmung des Unternehmens « und der»Reputation der Marke BMW - auch in der Presse (Ellinghaus 2011; zit.n.: Wagner 2013, S. 23). Wagner führt aus, dass z.B. das »Lab-Thema Urban Design und Mobility [...] nicht ergebnisoffen « diskutiert wurde, sondern »von vorneherein auf den Profitzweck des Unternehmens zugeschnitten « gewesen sei (Wagner 2013, S. 23). Es sollten »Mobilitäts- und Informationsdienstleistungen wie das Carsharing, die Vernetzung mit öffentlichen Verkehrsmitteln sowie die Anbindung der Fahrzeuge an die Kommunikationsmöglichkeiten des Internet [sic!] verstärkt eine Rolle spielen.«(Ebd.) 
Großkirchen« (ebd.) wachse »die Einsicht in Konzernen, Think Tanks, Beratungsunternehmen und Politik, dass sich manches ändern muss, damit grundsätzlich alles so bleiben kann wie bisher« (ebd., S. 14f.). So hätten schließlich auch Luc Boltanski/Chiapello (2003) in ihrer Analyse des >neuen Geistes des Kapitalismus herausgearbeitet, dass jener neue Geist von den abhängig Beschäftigten »die Fähigkeit zur Selbstorganisation« abfordere (und auch begünstige) und die klassischen Großorganisationen "immer weniger dazu in der Lage sind, sie ssozialpartnerschaftlich $\mathrm{zu}$ vereinnahmen« (Wagner 2013, S. 15). Insofern seien »innovative[...] Weisen der Befriedung bzw. der Vereinnahmung von potenziellem Widerstand« gefragt: »Hierher rührt die zunehmende Bereitschaft der Mächtigen in Wirtschaft und Politik, mit neuen Beteiligungsformen zu experimentieren.« (Ebd.)

Wagner selbst legt seiner Kritik an der Beteiligungsförderung durch Unternehmen und »konzernnahe[...] Stiftungen« (ebd., S. 14) ein Demokratieverständnis zugrunde, das Demokratie als "gesellschaftliche Organisationsform « versteht, »die ausgerichtet ist an der regulativen Idee einer Überwindung von Herrschaft« (ebd., S. 11). Er zitiert den Staatsrechtler Andreas Fisahn: "Dieses Verständnis geht hinaus über den in der Staatsrechtslehre herrschenden Begriff von Demokratie, der nur als Legitimation von - dann nicht in Frage gestellter - Herrschaft verstanden wird.« (Fisahn 2008, S. 62; zit.n.: Wagner 2013, S. 11f.) Demzufolge ginge es bei der Überwindung von Herrschaft

»nicht nur um die gleiche Teilhabe an den im engeren Sinne politischen Institutionen, sondern darüber hinaus am gesellschaftlich produzierten Reichtum, am Eigentum an den Produktionsmitteln und an den ökonomischen Entscheidungen.«(Wagner 2013, S. 12) ${ }^{65}$

Werde auf die »Erweiterung demokratischer Kontrollmöglichkeiten auf den Bereich der Produktion, des Privateigentums und der Wirtschaftslenkung« verzichtet,

»verliert der nach wie vor beliebte Slogan von der >Demokratisierung der Demokratie seine emanzipatorische Stoßrichtung und wird auch für jene Kräfte attraktiv, die nach neuen Wegen suchen, um die bestehende Eigentums- und Herrschaftsordnung zu erhalten und wenn möglich weiter auszubauen.« (Ebd.)

Für die Beteiligungsprojekte der unternehmensverbundenen Bertelsmann Stiftung stellt er deshalb fest, dass dies »[...] im Einzelnen durchaus seine Berechtigung haben« könnte (ebd., S. 147). Wenn aber »der gesellschaftliche Herrschaftszusammenhang dabei ausgeblendet« werde,

65 Wagner verweist hier auf Auseinandersetzungen mit dem Begriff der Demokratie bei Salomon (2012) und Wagner (2011). 
»können solche Projekte einen Beitrag zur Legitimation der bestehenden Verhältnisse leisten, ohne dass die Beteiligten das im Sinn gehabt haben müssen. Wie ökonomische Prozesse demokratisch gesteuert werden können, welche Eigentumsformen mit dem Anspruch gleicher politischer Beteiligung überhaupt vereinbar sind oder wie Betriebe demokratisch organisiert werden können: Diese entscheidenden Fragen für eine umfassende Demokratisierung der Cesellschaft werden überhaupt nicht berührt.« (Ebd., S. 147f.)

Wagner bezieht sich - vor allem am Beispiel seiner Analyse zu Mediations- und Dialogverfahren - auch auf Gramscis Überlegungen zu einer passiven Revolution (vgl. ebd., S. 138) (vgl. Kapitel 3.1.2). In Bezug auf »Konzernstiftungen« geht er davon aus, dass diese "großzügig bei der Bildung von bürgergesellschaftlichen Netzwerken helfen«, um dadurch »der Selbstorganisation der Bürger einen Rahmen und eine Richtung zu geben, die den Vorrang privater Profitinteressen vor dem Gemeinwohl nicht gefährdet.« (Ebd., S. 136) Es stellt sich die Frage, ob diese These auch auf politische Jugendbildungsprogramme übertragen werden kann bzw. inwiefern sie hier ggf. auch modifiziert werden muss.

Dass »[e]in großer Teil der deutschen Stiftungen [...] die Bedeutung des Bürgerengagements erkannt und in die Stiftungsaktivitäten aufgenommen hat « (Bundesverband Deutscher Stiftungen 2012b, S. 71), zeigt die Kurzstudie »Engagementförderung durch Stiftungen in Deutschland « - herausgegeben u.a. von der unternehmensverbundenen Körber Stiftung (vgl. BDS/Körber Stiftung 2010, S. 38). Der Bundesverband Deutscher Stiftungen hat zudem 2010 (vgl. ebd., S. 45) ein Forum Engagementförderung als »Plattform für den fachlichen Austausch zum Thema Bürgerengagement« initiiert (Bundesverband Deutscher Stiftungen 2012b, S. 71). In dem Forum wurde das Positionspapier »Mehr Bürgergesellschaft wagen! Stiftungen in der Engagementförderung« verabschiedet (vgl. Forum Engagementförderung 2012). Zu den zehn Autor*innen des Papiers gehören u.a. vier Vertreter*innen unternehmensnaher Stiftungen (Körber Stiftung, Robert Bosch Stiftung, Bertelsmann Stiftung und Vodafone Stiftung) (vgl. ebd.), was wiederum die Relevanz des Themas Bürgerengagement für unternehmensnahe Stiftungen zeigt.

Im öffentlichen Diskurs ist oftmals eine geradezu enthusiastische Begeisterung zum Thema Engagementförderung zu finden. Entgegengesetzt dazu hinterfragen Sozialwissenschaftler*innen eben jene Engagementförderung in Hinblick auf eine sogenannte Aktivierungsideologie, in der Bürger*innen zunehmend dazu angehalten werden, Aufgaben der staatlichen Wohlfahrtsproduktion zu übernehmen. Diese Diskussion kann hier nicht ausführlich dargestellt werden, weshalb nur beispielhaft auf die Analysen von Alexander Wohnig verwiesen sei, der sich mit der Bedeutung von »Engagement-Lernen in Politik, Gesellschaft und politischer Bildung « auseinandergesetzt hat (vgl. Wohnig 2014, 2017). Er schlussfolgert aus seiner Analyse der Engagementpolitik der Bundesregierung: 
»Die Aufgaben scheinen also klar verteilt. Der Staat, nicht mehr fähig für alle seine BürgerInnen aus dem Steueraufkommen Sorge zu tragen, soll Rahmenbedingungen für eine Selbsthilfe der BürgerInnen schaffen. Bürgerschaftliches Engagement erscheint hier als soziale Leistung, als unentgeltliche Unterstützung für den Staat.« (Ders. 2017, S. 124)

Weiterhin leitet er aus den Ausführungen des 2012 erschienenen ersten Engagementberichts der Bundesregierung (Titel: »Für eine Kultur der Mitverantwortung«, vgl. BMFSFJ 2012) ab, dass »[b] ürgerschafltiches Engagement und zivilgesellschaftliche Organisationen [...] im Rahmen des >Dritten Sektors einen Beitrag zur Wohlfahrtsproduktion leisten [sollen] (Wohnig 2017, S. 112). Es sei »eine Denkweise zu beobachten, die >Engagementく offen als dritte Säule des Sozialsystems begreift« (ders. 2014, S. 217). Basierend auf seiner Untersuchung der Engagementpolitik ordnet er die Forderungen nach verstärktem `Engagementlernen kritisch ein: »Im Kern soll Engagementlernen den >Sozialstaat der Zukunft sichern $<$ - und den klassischen Sozialstaat >entlasten`.« (Ebd., S. 219)

Insofern hinterfragt Wohnig auch Programme zum sogenannten Service Learning (in welchem Schüler*innen zu sozialem Engagement motiviert werden sollen) oder die Förderung von Engagementlernen im Rahmen von politischer Bildung (vgl. ebd., S. 220ff.) - beides Ansätze, die auch von unternehmensverbundenen Stiftungen vorangetrieben werden. So fördert die Freudenberg Stiftung seit vielen Jahren Forschung und Programme zum Service Learning (vgl. Ausführungen weiter unten). Die Bertelsmann Stiftung fördert >Engagementlernen politischen Bildung, z.B. über Unterrichtsmaterialien wie den »Mitmachheften«, die bei Schüler"innen aller Altersgruppen (Grundschule, Sekundarstufe 1 und 2) die »Bereitschaft zu zivilgesellschaftlichem Engagement [...] wecken« sollen (Lange 2011a, b, c). Wohnig deutet sie im Kontext des Versuchs,

»SchülerInnen in Engagementverhältnisse zu bringen, auch damit sie als Erwachsene später soziales Engagement zeigen, sich also im Sinne des staatlichen Wunsches nach mehr Engagement verhalten.« (Wohnig 2014, S. 224) ${ }^{66}$

Vor dem hier skizzierten Hintergrund deuten sich bereits viele (weiterführende) Fragen zur Beteiligungs- und politischen Bildungsförderung durch unterneh-

66 Wohnig bezieht sich hier auf den »Bericht zur Lage und den Perspektiven bürgerschaftlichen Engagements in Deutschland « (vgl. Alscher et al. 2009), an dem er zuvor den sstaatlichen Wunsch nach mehr Engagement « herausgearbeitet hat. Basierend auf der politikdidaktischen Lernprozessanalyse, die Wohnig am Beispiel des Modellprojekts »Soziale Praxis und Politische Bildung - Compassion \& Service Learning politisch denken« vorgenommen hat, plädiert er dafür, Engagementförderung als hegemoniales Muster im Kontext von Engagementförderungs-

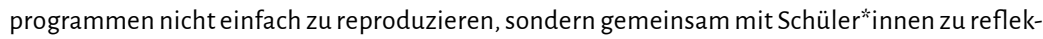
tieren (vgl. Wohnig 2017). 
mensnahe Stiftungen an: Werden hierbei gesellschaftliche Themen in einer bestimmten Weise >kanalisiert « und bearbeitet, die im Interesse von Unternehmen und deren Eigentümer*innen ist? Mit welchen Intentionen wird Bürger*innenengagement gefördert? Diese Fragen werden in der Fallstudie (vgl. Kapitel 5.2) wieder aufgegriffen. Zunächst soll ein Überblick über die Vielfalt an Aktivitäten unternehmensnaher Stiftungen sowie Stiftungen, deren Unternehmensnähe als ungeklärt gelten darf (vgl. dazu die Ausführungen in Kapitel 4.2.1), gegeben werden.

Hinsichtlich der thematischen Ausrichtung der Aktivitäten der in dieser Untersuchung identifizierten Stiftungen werden unterschiedliche Stoßrichtungen politischer Bildung erkennbar, unter anderem in Anknüpfung an:

- ökonomische Bildung: z.B. in Programmen der Joachim Herz Stiftung (»Youtube-Stars erklären Wirtschaft« oder das Forschungsprojekt »Schüler urteilen als Wirtschaftsbürger«, in dessen Rahmen auch Unterrichtsmaterialien entwickelt werden); ${ }^{67}$

- berufliche Bildung: z.B. bei der Robert Bosch Stiftung (Studie »Politische Bildung in der Berufsschule«) oder der Freudenberg Stiftung (z.B. »Business \& Bildung e.V.«). Die TUI Stiftung beschreibt ihren Leitgedanken wie folgt:

»Wir glauben, der Schlüssel zu einer tragfähigen Zukunft ist mehr denn je der Zugang zu Bildung und gesellschaftlicher Weiterentwicklung. Um die Chancengleichheit von jungen Menschen in allen Ländern der Europäischen Union zu verbessern, fördern wir Projekte rund um das Thema >]unges Europa mit den Schwerpunkten Bildung, Ausbildung sowie individuelle und berufliche Entwicklung. [...]« (TUI Stiftung 2017)

Die Heinz Nixdorf Stiftung fördert (gemeinsam mit der Stiftung der Deutschen Wirtschaft) die Initiative »Herausforderung Unternehmertum $«,{ }^{68}$ mit der ein Beitrag zur Stärkung des unternehmerischen Denkens und Handelns in Deutschland« geleistet werden soll (Heinz Nixdorf Stiftung 2017d). Stipendiat*innen »erhalten« hierbei

»die Chance, unternehmerische Qualifikationen zu erwerben und ihre Projektund Cründungsideen in die Tat umzusetzen. [...] Das Spektrum reicht von ge-

67 Alle nachfolgend genannten Programme finden sich mit Quellennachweis und Kurzbeschreibung im Anhang. Für bessere Lesbarkeit wurde deshalb nachfolgend darauf verzichtet, zu jedem Programmname die Quelle im Fließtext zu nennen.

68 Die Heinz Nixdorf Stiftung führt diese Initiative auf ihrer Website unter dem Stichwort »Förderprojekte demokratisches Staatswesen«(Heinz Nixdorf Stiftung 2017d). 
winnorientierten Unternehmensgründungen über Vorhaben im Sinne eines >Social Entrepreneurship bis zu gemeinnützigen Projekten« (ebd.);

- historische Bildung: z.B. bei der Körber Stiftung (z.B. »History Campus«, »history@debate«, »Geschichtswettbewerb des Bundespräsidenten«);

- soziale Bildung: z.B. bei der Freudenberg Stiftung (Service-LearningProgramm »Lernen durch Engagement «).

Interessant ist, dass das letztgenannte Programm nun auch verstärkt im Kontext beruflicher Bildung verortetet wird. So fördert die Freudenberg Stiftung gemeinsam mit der Deutschen Bahn Stiftung »Berufene Helden - Lernen durch Engagement für Chancen im Beruf « (Deutsche Bahn Stiftung 2017). Dabei wird »Lernen durch Engagement< (Abk. LdE, engl. Service-Learning)« als »eine Lehr- und Lernform« verstanden,

»die gesellschaftliches Engagement von Schülerlnnen mit fachlichem Lernen verbindet. Das Engagement der SchülerInnen wird dabei im Unterricht geplant, reflektiert und mit dem Erwerb spezifischer Kompetenzen und Fähigkeiten um Ausbildungsreife zu erlangen verknüpft.« (Ebd.)

Die Verbindung von ökonomischer und politischer Bildung wird aktuell kontrovers diskutiert. ${ }^{69}$ Gerade private Akteure (wie Unternehmen und unternehmensnahe Stiftungen) sehen sich hier dem Vorwurf ausgesetzt, einseitige, sarbeitgebernahe< Bildung zu vermitteln (vgl. Möller/Hedtke 2011; Engartner 2015). Ob ökonomische Bildungsangebote von unternehmensnahen Stiftungen prinzipiell einseitig sind, ist bisher nicht untersucht. In Programmbeschreibungen unternehmensnaher Stiftungen lässt sich häufig das Ziel finden, dass Jugendliche etwas über >Unternehmensgründung und -führungく lernen sollen, womit eher die Arbeitgeber-, als die Arbeitnehmerperspektive angesprochen scheint. Beispielhaft sei hier eine Projektbeschreibung der Joachim Herz Stiftung genannt:

»Die Teilnahme an dem computergestützten Unternehmensplanspiel EcoStartup hilft den Schülern, die von angehenden Unternehmern benötigten Kompetenzen zu erwerben. Durch den Einsatz von EcoStartup werden den Schülern grundlegende betriebswirtschaftliche Kenntnisse vermittelt. In Teams gründen sie ihre eigenen Unternehmen, entwickeln auf Basis der Geschäftsidee den konkreten Businessplan und setzen ihn in den ersten Ceschäftsjahren um. Dabei stehen sie im Wettbewerb mit weiteren Jungunternehmern und lehren etablierte Betriebe [sic!] das Fürchten. Durch EcoStartup werden die betrieblichen Abläufe

69 Einen Überblick über die aktuelle Debatte geben Engartner/Krisanthan (2017) in ihrem Band »Wieviel ökonomische Bildung braucht politische Bildung?«. 
und Zusammenhänge der Unternehmensgründung und -führung sowie deren Einbettung in ein individuelles Unternehmensumfeld deutlich gemacht. Neben dem betriebswirtschaftlichen und gründungsspezifischen Fachwissen werden auch Sozialkompetenz und Konfliktfähigkeit gefördert. (Joachim Herz Stiftung 2017a)

Das Zitat macht deutlich, wie Jugendliche sich in Wettbewerbsverhalten einüben und unternehmerische Leitbilder verinnerlichen sollen. Es stellt sich die Frage, ob ökonomische Bildung auf diese Einübung zielen oder nicht vielmehr diese gesellschaftlichen Anforderungen auch kritisch hinterfragen sollte. Die Joachim Herz Stiftung hat weiterhin »[i]n Zusammenarbeit mit bekannten YouTubern [...] insgesamt 40 Videos zum Thema Wirtschaft« produziert - genau genommen zur Frage »Was ich noch nie über Wirtschaft wissen wollte'« (dies. 2017b). Dabei würden "grundlegende ökonomische Zusammenhänge mit direktem Aha-Effekt« erklärt zu Fragen wie:

»Welche Produkte will ich kaufen? Welcher Beruf passt zu mir? Will ich für mein Alter privat vorsorgen? Die Jugendlichen erkennen, dass sie in der Rolle des Konsumenten, des Erwerbstätigen oder als Wirtschaftsbürger wirtschaftlich agieren und so die Wirtschaft mitgestalten.« (Ebd.)

Damit findet sich in dem Programm recht offensichtlich auch das vorherrschende Bild des eigenverantwortlichen Konsumenten wieder, das in der politischen Bildung z.T. kritisch betrachtet wird (vgl. Eis/Moulin-Doos 2015, S. 16, 20).

Lobbyismus an Schulen wird mittlerweile gesellschaftlich breiter diskutiert und auch kritisiert. ${ }^{70}$ Inwiefern auch außerschulische Jugendbildung als Anknüpfungspunkt für Lobbyismus genutzt wird, steht dagegen bisher kaum zur Debatte. Dafür, dass es auch hier Anknüpfungspunkte für Imageförderungsmaßnahmen von Unternehmen gibt, lassen sich auch mit wenig Rechercheaufwand Hinweise finden. So wurde z.B. in Kooperation von der Schwarzkopf-Stiftung Junges Europa, dem Europäischen Jugendparlament und der innogy Stiftung (des Energiekonzerns innogy, einer Tochterfirma von RWE) das Projekt »Young Energy for Europe« 2013/2014 organisiert:

»Im Rahmen eines pan-europäischen Plenums zum Thema Energie lernten sie [junge Menschen; Anmerk. v. A.H.], sich aktiv mit gesellschaftlichen Fragen auseinander zu setzen und Visionen zur Gestaltung Europas zu entwickeln.« (RWE Stiftung 2017a)

70 So gab es z.B. eine kontroverse Debatte zu einem Cesetzentwurf des Landes Hessen, Werbung an Schulen zu verbieten (vgl. RT 2017). 
Inwieweit in solchen Programmen auch Lobbyismus betrieben wird, also z.B. bestimmte Positionen zur Energiewende gezielt vermittelt werden, müsste näher untersucht werden. Bei der Schwarzkopf-Stiftung Junges Europa sind weitere Beispiele für die Kooperation mit Unternehmen zu finden. ${ }^{71}$

Zahlreiche Stiftungsprogramme in der politischen Bildung zielen auf Kompetenzförderung, die auf den Stiftungs-Websites nicht selten in Verbindung mit dem Erwerb von beruflichen Qualifikationen betrachtet werden. Beispielhaft seien genannt:

- »Jugend debattiert (gefördert durch die Stiftung Mercator, Gemeinnützige Hertie Stiftung, Robert Bosch Stiftung, Heinz Nixdorf Stiftung) ${ }^{72}$ »stärkt« bei Schüler*innen ab der 5 . Klasse »kommunikative Fähigkeiten, verbessert die politische Urteilsbildung, gibt mehr Sicherheit im Auftreten bei Prüfung und Bewerbung, vernetzt, bundesweit und vielfältig « (Jugend debattiert 2017b). ${ }^{73}$

- Beim »European Youth Parliament« (gefördert durch die Stiftung Mercator und die Schwarzkopf-Stiftung Junges Europa $)^{74}$ sollen die Teilnehmenden auch »Schlüsselqualifikationen für die berufliche Zukunft, wie etwa internationale Teamarbeit, Sprachen und soziale Kompetenz«, erwerben (SchwarzkopfStiftung Junges Europa 2017a).

- Die Körber Stiftung

»[engagiert sich] [m]it dem Fokusthema Digitale Mündigkeit [...] für mündiges Handeln und eine starke Zivilgesellschaft im digitalen Zeitalter. Sie setzt sich für die Vermittlung umfassender digitaler Kompetenzen ebenso ein wie für eine gesamtgesellschaftliche Debatte über die Cestaltung des digitalen Wandels.« (Körber Stiftung 2017a)

Weiterhin sind zahlreiche Stiftungsprogramme zur europapolitischen Bildung und zur Extremismusprävention zu finden. So hat beispielsweise die SchwarzkopfStiftung Junges Europa den Schwerpunkt auf diese Themen:

71 Der aktuelle Jahresbericht verweist unter dem Stichwort »Partner und Sponsoren« u.a. auf die Berliner Sparkasse, Deloitte, Stroer, Ernest \& Young, Deutsche Telekom (vgl. SchwarzkopfStiftung Junges Europa 2016).

72 Zu den Partnern gehören außerdem: die Kultusministerkonferenz, die Kultusministerien und Parlamente der 16 deutschen Länder, Schirmherr ist der Bundespräsident (vgl. Jugend Debattiert 2017a).

73 Vgl. hierzu kritisch: Wolf 2011.

74 Zu den Partnern gehören außerdem: die Unternehmen Deutsche Telekom AC und ECO Schulte $\mathrm{CmbH} \&$ Co. KG, das Auswärtige Amt, die Vertretung der Europäischen Kommission in Deutschland und das EU-Programm Erasmus+ (vgl. Europäisches Jugendparlament 2017). 
»Stiftungszweck ist die Förderung der Entwicklung junger Menschen zu politisch bewussten und verantwortungsbereiten Persönlichkeiten mit dem Ziel der Stärkung des europäischen Gedankens, der gesamteuropäischen Völkerverständigung und der Bekämpfung von Rechtsextremismus, Antisemitismus und Rassismus.« (Schwarzkopf-Stiftung Junges Europa 2017b)

Weitere Formate zur Europabildung sind zu finden bei der

- BMW Stiftung Herbert Quandt (Dialogreihe »Europa in Vielfalt vereint«, Diskussionsreihe »Inseldialoge - Europa in der Welt«),

- Gemeinnützigen Hertie-Stiftung (Projekt »Nächste Generation: Europäer?!«),

- Robert Bosch Stiftung (»Schulbrücke Europa«),

- Stiftung Mercator (Förderung der Plattform »Europa verstehen«).

Extremismuspräventions-Programme finden sich z.B. bei der

- Freudenberg Stiftung (z.B. Unterstützung des Online-Portals »Netz gegen Nazis«)

- oder bei der Robert Bosch Stiftung (Programm »Starke Lehrer - Starke Schüler«, »Jugend gegen Extremismus« oder »Rückgrat - Gegen Rechtsextremismus«).

Es lassen sich bestimmte Schwerpunktsetzungen der Stiftungen ausmachen, wie eben das Thema Europa bei der Schwarzkopf-Stiftung Junges Europa, das Thema Wirtschaftsbildung (in starker Verbindung mit gesellschaftspolitischen Themen) bei der Joachim Herz Stiftung oder der Fokus auf >politikferne< Jugendliche bei der Robert Bosch Stiftung (vgl. Kapitel 4.3). Einige der genannten Stiftungen zeichnen sich durch ein breites Zielgruppen- und damit einhergehend auch breites Formatspektrum aus. Beispielhaft sei die RBS genannt, die Schüler*innen (»Dialog macht Schule«), Jugendliche außerhalb der Schule (»Lernort Stadion«), Kindererzieher*innen (»Partizipation in der Kita«), Lehrkräfte (»Starke Lehrer - starke Schüler«), Journalist*innen (»Journalistenprogramme«) oder die weitere Bevölkerung (»Actors of Urban Change«, »Neulandgewinner. Zukunft erfinden vor Ort«) anspricht.

Auch sei auf die vielfältigen Politikberatungsaktivitäten unternehmensnaher Stiftungen hingewiesen, die sich z.T. an der Schnittstelle zur politischen Bildung befinden. Es kommt gewissermaßen auf den Standpunkt der Betrachtung an: Denn wenn z.B. im Rahmen von Diskussionsreihen Politiker*innen eingeladen sind und gleichzeitig ein öffentliches Publikum angesprochen wird, kann eine solche Diskussionsreihe unterschiedliche Funktionen erfüllen - eben Politikberatung oder/und politische Bildung. 
Der »Monitor politische Bildung« ordnet auch solche Veranstaltungen der politischen Bildung $\mathrm{zu}$, die sich ausschließlich an >Entscheidungsträger*innen richten, also auch als Politikberatungsaktivitäten gelten können: So werden z.B. die Formate der Körber Stiftung »Bergedorfer Gesprächskreis« oder das »KörberNetzwerk Außenpolitik« genannt (Lange 2010, S. 129). Bei Ersterem »diskutieren hochrangige internationale Politiker und Experten in kleiner und vertraulicher Runde Grundfragen deutscher und europäischer Außen- und Sicherheitspolitik« (Körber Stiftung 2017b), das Zweite »bringt regelmäßig einen festen Kreis jüngerer Außenpolitiker ins Gespräch mit internationalen Entscheidungsträgern« (dies. 2017c). Die Einordnung solcher Formate als politische Bildung sollte nicht dazu verleiten, Funktionen der Politikberatung und Elitennetzwerkbildung $\mathrm{zu}$ übersehen. Gerade aus politikwissenschaftlicher Perspektive gilt es, hier genauer hinzuschauen.

Politikberatungsformate lassen sich auch bei der BMW Stiftung Herbert Quandt finden. ${ }^{75}$ Ihre Veranstaltungen sind nicht für alle zugänglich, sondern »[t]eils öffentlich, teils auf Einladung« (BMW Stiftung Herbert Quandt 2016). Die Stiftung benennt sogar »internationale Führungspersönlichkeiten ${ }^{76}$ als die Hauptzielgruppe ihrer gemeinnützigen Stiftungstätigkeiten und ist damit dezidiert elitär ausgerichtet. Begründet wird dies darüber, dass Führungspersönlichkeiten

»[a]ls Multiplikatoren, mit ihrem Einfluss und Erfahrungsschatz [...] einen wichtigen Beitrag zu einer solidarischen Cesellschaft leisten [können]. Zum einen, indem sie im Beruf immer auch die gesellschaftlichen Rückkoppelungen ihres Handelns berücksichtigen. Zum anderen können sie dem Auseinanderdriften moderner Cesellschaften entgegenwirken, wenn sie ihren gewohnten Handlungsraum verlassen und Cemeinsinn zeigen.«(BMW Stiftung Herbert Quandt 2017a)

Auch die Nixdorf Stiftung führt z.B. das Programm »Young Leaders for Europe« unter dem Stichwort >Förderprojekte demokratisches Staatswesen (vgl. Nixdorf Stiftung 2017a).

Schließlich sei noch erwähnt, dass unternehmensnahe und private Stiftungen andere Einrichtungen, wie Governance Schools oder weitere Stiftungen, fördern und auch auf diese Weise Einfluss auf dem Feld der politischen Bildung geltend machen: So fördert z.B.

75 Als Beispiel kann der BMW Foundation European Table genannt werden (vgl. BMW Stiftung Herbert Quandt 2017b).

76 Auf der Website steht zu lesen: »Mit unserer Arbeit wenden wir uns besonders an eine Zielgruppe: internationale Führungspersönlichkeiten.«(BMW Stiftung Herbert Quandt 2017a) 
- die Stiftung Mercator die NRW School of Governance,

- die Nixdorf Stiftung die Ludwig-Ehrhart-Stiftung ${ }^{77}$ und die Stiftung politische und christliche Jugendbildung, ${ }^{78}$

- $\quad$ die Freudenberg Stiftung die Amadeu Antonio Stiftung. ${ }^{79}$

$\mathrm{Zu}$ den Kooperationspartnern der Stiftungen konnte im Rahmen dieser Studie nicht recherchiert werden, jedoch verweisen die Programmbeschreibungen auf Kooperationen (in der politischen Bildung) u.a. mit der bpb, den Landeszentralen für politische Bildung, den parteinahen Stiftungen, Schulen und Ministerien, mit EUInstitutionen, anderen Stiftungen und Unternehmen. ${ }^{80}$

Die Bertelsmann Stiftung, RBS und Stiftung Mercator sind zudem im Beirat der Transferstelle politische Bildung vertreten (vgl. Transferstelle politische Bildung 2017b). Diese wird von der bpb finanziert, stellt Informationen zu Forschungsarbeiten der politischen Bildung bereit und dient »der Vernetzung und dem Austausch von Praxis, Wissenschaft und Politik« (dies. 2017a). Der Beirat "soll sicherstellen, dass die Arbeit der Transferstelle am tatsächlichen Bedarf der Praxis politischer Bildung ausgerichtet ist « (dies. 2017c). Unternehmensnahe Stiftungen spielen in der politischen Bildung zudem eine Rolle als Auftraggeber von Forschung. ${ }^{81}$

77 Die Ludwig-Erhard-Stiftung wurde von Altbundeskanzler Ludwig Erhard 1967 gegründet; er "gab ihr die Aufgabe, für freiheitliche Crundsätze in Wirtschaft und Politik einzutreten und die Soziale Marktwirtschaft wachzuhalten und zu stärken« (Ludwig Erhard Stiftung 2017).

78 Die Stiftung hat keine Website, laut Angabe auf der Website der Nixdorf Stiftung ist sie »ein überparteiliches und überkonfessionelles Bildungswerk. Ihr Ziel ist, jungen Menschen die ideellen Voraussetzungen der freiheitlichen Cesellschafts- und sozialen Marktwirtschaftsordnung zu vermitteln. Dabei stützt sie sich auf die christliche Sozialethik und Soziallehre und wendet sich gegen Rechtsextreme, Linksextreme und islamistisch-fundamentalistische Ideologien. Zu den Lernzielen gehört die Vermittlung von Toleranz. [...] « (Nixdorf Stiftung 2017b)

79 Die Amadeu Antonio Stiftung wurde 1998 gegründet. Ihr »Ziel« sei es, »eine demokratische Zivilgesellschaft zu stärken, die sich konsequent gegen Rechtsextremismus, Rassismus und Antisemitismus wendet« (Amadeu Antonio Stiftung 2017).

80 Exemplarisch und in Ergänzung der bisherigen Ausführungen, in denen schon einige Kooperationspartner genannt wurden, sei hingewiesen auf die folgenden Partner: Die bpb ist Kooperationspartner der Robert Bosch Stiftung (z.B. im Programm »Dialog macht Schule«). Die parteinahe Heinrich-Böll-Stiftung kooperierte 2014 u.a. mit der Bertelsmann Stiftung bei der internationalen Konferenz »Cities of Migration. Eine Agenda des Wohlstands für alle« (vgl. Heinrich Böll Stiftung 2017). Die Landeszentrale für politische Bildung Hamburg kooperierte mit der Körber Stiftung (und weiteren Partnern) für die »Tage des Exils« (vgl. Körber Stiftung 2017d). Die Freudenberg Stiftung kooperiert mit dem Bildungsministerium Thüringen bei der Umsetzung des Programms »Service-Learning - Lernen durch Engagement« (vgl. Freudenberg Stiftung 2017c).

81 So weist Becker in ihrer Bestandsaufnahme empirischer politischer Bildungsforschung darauf hin, dass " 35 bis $32 \%$ der Studien [...] im direkten Auftrag von Ministerien oder Stiftungen erstellt [wurden] « (Becker 2011a, S. 15). Unter den Drittmittelgebern finden sich neben den par- 
Die Angaben zu Stiftungsbudgets wurden im Rahmen dieser Untersuchung nicht erhoben, da i.d.R. nur das Jahresgesamtbudget und nicht das Budget für politische Bildungsaktivitäten angegeben wird. ${ }^{82}$ Nimmt man den bpb-Haushalt (2015: rund 42 Millionen) als Vergleichshaushalt (vgl. Bundeszentrale für politische Bildung 2016), so ist das Stiftungsbudget einiger Stiftungen dennoch beeindruckend: die Stiftung Mercator hat rund 114 Millionen, die Bertelsmann Stiftung rund 156 Millionen und die Bosch Stiftung rund 106 Millionen zur Verfügung. ${ }^{83}$ Wieviel genau davon in politische Bildungsaktivitäten fließt, müsste eigens erhoben werden.

Bezüglich der Einschätzung des Einflusses auf den (politischen wie auch allgemeinen) Bildungsbereich ist schließlich noch wichtig, den Arbeitsmodus der Stiftungen (operativ/fördernd) zu beachten. So sind einige nur operativ tätig, d.h., sie kooperieren zwar ggf. in Programmen, aber fördern keine anderen Initiativen, sondern führen nur selbst konzipierte Projekte durch und nehmen somit eine starke Steuerung vor. ${ }^{84}$ Eine reine operative Tätigkeit wird in der Fachöffentlichkeit z.T. kritisch beurteilt. Die Politikwissenschaftlerin Gesine Schwan hat sich so z.B. für eine Begrenzung operativer Stiftungen ausgesprochen: Sie sollten »allenfalls 10 oder 20 Prozent für operative Zwecke« verwenden und den »Rest, um wirklich Vielfalt und Zivilgesellschaft zu fördern« (Kocka et al. 2011, S. 187). Ausschließlich operativ tätig sind z.B. die Bertelsmann Stiftung, die Körber Stiftung, die Joachim Herz Stiftung und die BMW Stiftung Herbert Quandt.

Dass Stiftungsprogramme - wenig überraschend - auch elitär ausgerichtet sein können, zeigen einige der bisherigen Ausführungen: So kann z.B. das Europäische Jugendparlament mit der Zielgruppe Studierende, die auf Englisch kommunizieren können, als eher elitär gelten. >Benachteiligte Jugendlicheく werden im Rahmen von Engagement-Programmen und politischen Bildungsprogrammen jedoch auch angesprochen - nicht nur von der RBS (vgl. Kapitel 4.3) und der hier im Rahmen einer anonymisierten Fallstudie betrachteten Unternehmensstiftung (vgl. Kapitel 5), sondern auch von anderen Stiftungen.

teinahen Stiftungen auch unternehmensnahe Stiftungen - wie z.B. die Bertelsmann Stiftung (vgl. ebd., S. 16). Die Deutsche Kinder-und Jugendstiftung hat z.B. einen eigenen Arbeitsbereich »Entwicklung und Evaluation «(vgl. Deutsche Kinder-und Jugendstiftung 2017d). Vgl. weiterhin dazu die Ausführungen zu den von der RBS beauftragten Evaluationen in Kapitel 4.3.3.

82 Die Erhebung der Höhe dieses Anteils bedürfte umfangreicher Recherchearbeiten, die für diese Arbeit nicht geleistet werden konnten. Problem ist zudem, dass die Stiftungen selbst oft nur grobe Angaben machen -z.B. Ausgaben im Bereich Wissenschaft, Bildung etc. Nur wenige Stiftungen geben die Ausgaben pro Programm/Projekt an (so z.B. die Stiftung Mercator).

83 Hinzu kommt bei diesen Stiftungen, dass sie noch weitere Einrichtungen (z.B. Forschungsinstitute) gegründet haben, deren Budgets hier nicht eingerechnet sind.

84 Natürlich haben auch fördernde Stiftungsaktivitäten einen operativen Charakter, wie Adloff hervorhebt, da auch eine Entscheidung über die Förderung getroffen wird (vgl. Adloff 2010, S. 390). 
Dabei scheinen die Themen soziale Ungleichheit und Politikverdrossenheit generell stärker ins Bewusstsein der Stiftungswelt gerückt zu sein: Der Bundesverband Deutscher Stiftungen hat in einem Stiftungsreport zum Thema »Wie Stiftungen den sozialen Zusammenhalt stärken« (Bundesverband Deutscher Stiftungen 2012b) als zwei von drei Problemfeldern »Soziale Ungerechtigkeiten« und »Politikverdrossenheit « definiert (ebd., S. 10). Zum Erstgenannten führt er u.a. aus, dass »[...] [d]ie ungleiche Verteilung von Teilhabechancen [...] die Akzeptanz des Systems [schwächt].«(Ebd.) Stiftungsengagement könne hierbei »zu mehr Chancengerechtigkeit « führen, wobei »vor allem ein Aspekt bedeutsam [ist]: Bildung« (ebd., S. 11). Dabei müsse man auch den Ansprüchen der Wissensgesellschaft gerecht werden:

»Im digitalen Zeitalter hat sich auch der Bildungsanspruch verändert. Die reine Vermittlung von Faktenwissen rückt in den Hintergrund. In der Wissensgesellschaft, die durch hohe Spezialisierung und ständige Abrufbarkeit von Wissen gekennzeichnet ist, sind vor allem methodische Fähigkeiten gefragt. Schüler müssen heute in erster Linie wissen, wo sie die Informationen, die sie brauchen, finden. Sie sollen Wissen transferieren und vernetzen können. Von großer Bedeutung ist der Erwerb sogenannter Soft Skills wie Kommunikationsfähigkeit. Ferner erzeugt ein moderner Bildungsansatz ein naturwissenschaftlich-technisches Verständnis und fördert ein kulturelles Bewusstsein. All das jeweils entlang der ganzen Bildungskette - von pädagogischer Frühförderung bis ins Studium. Dazu ist ein Mentalitätswandel der im Bildungssektor Tätigen notwendig. [...] In Anbetracht der Schwerfälligkeit des föderalen Bildungssystems und einer konservativen Grundhaltung vieler Bildungseinrichtungen, gehen diese neuen Impulse häufig von Stiftungen aus.« (Ebd., S. 47)

Als ein »Mittel gegen Politikverdrossenheit« wird wiederum »Bürgerbeteiligung« (ebd., S. 62) gesehen, wobei Stiftungen »auch diejenigen erreichen [wollen], die nicht in der Mitte der Gesellschaft stehen« (ebd., S. 63). ${ }^{85}$ Auch bei den hier im Fokus stehenden unternehmensnahen Stiftungen sind solche Projekte zu finden. So gehören beispielsweise »Jugendliche mit Migrationshintergrund, die keinen Ausbildungsplatz finden, Kinder aus Zuwandererfamilien, die ohne ausreichende Deutschkenntnisse in der Schule nicht zurechtkommen [...]«, zu den zwei (von vier) Hauptzielgruppen der Freudenberg Stiftung (Freudenberg Stiftung 2017a). Die Stiftung »erprobt [...] Handlungsansätze im Feld der Migration/Integration,

85 Der Zusammenhang von sozialer Ungleichheit und politischer Partizipation wird dabei in dem BDS-Report differenziert besprochen und z.B. festgestellt, dass das »Prekariat« heute kaum noch politisch repräsentiert ist. »Bei ökonomischer Benachteiligung (die schon vorher als problematisch für die Systemakzeptanz dargestellt wurde) wird unter Bezug auf Nancy Fraser festgestellt, dass hier »Umverteilung die angemessene Antwort« sei (Bundesverband Deutscher Stiftungen 2012b, S. 72f.). 
Jugend zwischen Schule und Beruf sowie der Demokratischen Kultur [...]« und ihre »Vorhaben zielen generell auf [...] soziale Inklusion oder Integration« (ebd.). ${ }^{86}$ Die Freudenberg Stiftung war Mitinitiatorin des Programms »Ein Quadratkilometer Bildung«, dessen

»Programmziel seit dem Start 2007 ist, innerhalb von zehn Jahren zu erproben und modellhaft zu zeigen, wie Bildungsförderung von Kindern und Jugendlichen im Einzugsbereich ausgewählter Brennpunktgrundschulen gelingen kann.« (Bundesverband Deutscher Stiftung 2012a, S. 40)

Das Programm »wird durch einen Stiftungsverbund fachlich begleitet «, zu dem auch die BMW Stiftung Herbert Quandt gehört (ebd.). »Demokratie leben« bildet dabei einen Schwerpunkt, der

»für das Programm Ein Quadratkilometer Bildung [heißt], dass Kinder und Jugendliche sich als selbstwirksam erfahren, demokratisch sprechen und handeln können und dass sie Kitas, Kinder- und Jugendeinrichtungen, Schulen und den Stadtteil als Orte der Demokratie erleben. Grundlegend für diesen Schwerpunkt ist der unmissverständliche Bezug auf die Kinderrechte.« (Ein Quadratkilometer Bildung 2017)

Auch die Schwarzkopf-Stiftung Junges Europa förderte z.B. politische Bildungsseminare für Teilnehmer*innen des Schüler*innenstipendiums der Roland-BergerStiftung. Im Jahresbericht von 2013 heißt es dazu:

»Das Schülerstipendium der Roland-Berger-Stiftung fördert begabte Jugendliche aus sozial benachteiligten Familien. Schon seit 2009 arbeitet die SchwarzkopfStiftung eng mit der Roland-Berger-Stiftung zusammen und gestaltet politische Bildungsseminare für die Schülerstipendiaten. Junge Seminartrainer der Schwarzkopf-Stiftung erarbeiteten im vergangenen Jahr mit über 100 Stipendiaten in beinahe allen Bundesländern Inhalte zu Themen wie Demokratie, Migration und Integration sowie Grundlagen zur Europäischen Union. [...]« (Schwarzkopf-Stiftung 2013, S. 19)

Die Vodafone Stiftung ist nur mit einzelnen Programmen im Bereich politischer Bildung aktiv, darunter keine Programme für >benachteiligte Jugendlicheく. Jedoch ist ihr Stiftungsprofil insgesamt stark gesellschaftspolitisch ausgerichtet ${ }^{87}$ und die

86 Für einen ausführlicheren Einblick in die Arbeit der Freudenberg Stiftung: vgl. Gerber 2006.

87 Die Stiftung »initiiert [...] Programme mit dem Ziel, Impulse für den gesellschaftlichen Fortschritt zu geben, die Entwicklung einer aktiven Bürgergesellschaft anzustoßen und gesellschaftspolitische Verantwortung zu übernehmen. Das Förderprofil steht unter dem Leitmotiv >Menschen fördern. Ideen fördern < und konzentriert sich auf die Bereiche Bildung, sozialer Aufstieg und gesellschaftlicher Zusammenhalt« (Vodafone Stiftung 2017a). 
Förderung >benachteiligter Jugendlicher $<$ wird über diverse Bildungs- und Sozialprogramme vorgenommen. ${ }^{88}$

Auch die im Feld der politischen Bildung sehr umtriebige Bertelsmann Stiftung hat im Rahmen ihres Jugendbeteiligungsprogramms "jungbewegt « ab 2012 für eineinhalb Jahre ein Teilprojekt für `benachteiligte Jugendlicheく realisiert: »GE$\mathrm{Be}$ - Gesellschaftliches Engagement Benachteiligter in der Offenen Kinder- und Jugendarbeit fördern « (vgl. Sturzenhecker 2016a, b). ${ }^{89}$ Es wurde »mit sieben Einrichtungen der Offenen Kinder- und Jugendarbeit « kooperiert, als Zielgruppe sollte das Programm

»eben jene Jugendlichen erreichen, die im Alltag des Jugendhauses, aber auch allgemein, nur selten die Chance bekommen, etwas mitzubestimmen und mitzugestalten. Solche benachteiligten Kinder und Jugendlichen haben nur sehr begrenzt die Möglichkeit, sich überhaupt mit ihren Meinungen und Anliegen öffentlich zu zeigen. Wenn sie sich zeigen und ihre Stimme erheben, wird das von Gesellschaftsmitgliedern oft als deviant, also abweichend, wahrgenommen.« (Schwerthelm/Sturzenhecker 2016, S. 11)

In dem Programm wollte man »neue Arbeitsweisen und Methoden zur Förderung gesellschaftlichen Engagements entwickeln und erproben«(ebd.). Dazu sollten die

»beteiligten Einrichtungen [...] den benachteiligten Kids (wieder) näherkommen und sie dabei unterstützen, neue Handlungsweisen zu entdecken, um in der Cesellschaft mitbestimmen und mitgestalten zu können.« (Ebd.)

Es wird betont, dass man damit "genau dem Auftrag der Kinder- und Jugendarbeit nach $\$ 11$ SGB VIII« folge, der darauf abzielt, »dass sich die Kinder und Jugendlichen in der Jugendarbeit Selbstbestimmung, gesellschaftliche Mitverantwortung und soziales Engagement aneignen « (ebd.). Tatsächlich steht in dem Gesetz nichts von »Machtkritik«, wie sie z.B. aktuell wieder verstärkt von der politischen Jugendbildung eingefordert wird (vgl. u.a. Frankfurter Erklärung 2015). Im Projekt GEBe sollten »die Themen der Jugendlichen [...] zum Ausgangspunkt für Ansätze gesellschaftlichen Engagements« genommen werden, die Angebote »im Dialog gemeinsam mit den Jugendlichen entwickelt und durchgeführt « werden (Schwerthelm/Sturzenhecker 2016, S. 12). Auch wenn auf das Programm GEBe im Rahmen

88 Zum Beispiel im »buddY-Programm«: »In Projekten, die individuell auf die Schulen zugeschnitten sind, übernehmen Schüler etwa Patenschaften für jüngere Mitschüler, helfen anderen beim Lernen, setzen sich als Streitschlichter ein oder engagieren sich in sozialen Einrichtungen. «(Vodafone Stiftung 2017b)

89 Aus dem Projekt sind zwei Publikationen hervorgegangen (vgl. Sturzenhecker 2016a, b). Diese wurden im Bertelsmann Verlag publiziert und sind käuflich zu erwerben; eine kostenfreie oder reduzierte Version ist nicht verfügbar. 
dieser Arbeit nicht näher eingegangen werden kann, so verweisen die wenigen Auszüge aus der Programmbeschreibung eindrücklich darauf, dass die nachfolgenden, vertiefter analysierten Fälle in ihrer Ausrichtung an Themeninteressen sbenachteiligter Jugendlicher keine Einzelfälle sind.

Ein Beitrag des Deutschlandfunks weist 2015 darauf hin, dass sich unternehmensnahe Stiftungen zwar einerseits immer wieder dem Vorwurf ausgesetzt sehen, eine neoliberale Bildungspolitik zu betreiben (vgl. Laak/Schulz 2015). Andererseits wird festgestellt, dass sich die Vodafone Stiftung wie "auch Bertelsmann, Bosch und Telekom für traditionell linke Themen ein[setzen], wie die Förderung der frühkindlichen Bildung, bessere Integration von Migranten, [sic!] und Inklusion « (ebd.). So würden »sich die jüngeren Veröffentlichungen« der Vodafone Stiftung »gar nicht neoliberal [lesen] « (ebd.). Der Geschäftsführer Mark Speich erklärt in einem Interview:

»Wenn es neoliberal ist, dass man sich dafür einsetzt, dass Kinder aus sozial schwachen Familien gelingenden Bildungsaufstieg erleben können, dann würde ich mich zu dem Begriff auch ganz offensiv bekennen, also insofern ist dieser schillernde Begriff etwas schwierig.«(Ebd.)

In der philanthropie-kritischen (politikwissenschaftlichen) Fachliteratur lässt sich das Engagement unternehmensnaher Stiftungen als Teil einer Strategie verorten, mit der die sozial rauen Seiten kapitalistischer Systeme entschärft, jedoch nicht substantiell abgebaut werden sollen. So befinden Robert F. Arnove/Pinede für die US-amerikanischen »)Big Three< Foundations« (»Carnegie, Rockefeller, and Ford Foundations«):

»These foundations claim to attack the roots causes of the ills of humanity; however they essentially engage in ameliorative practices to maintain social and economic systems that generate the very inequality and injustices they wish to correct.« (Arnove/Pinede 2007, S. 289)

Linsey McGoey befindet zudem in ihrer Analyse großer US-amerikanischer Stiftungen (mit Schwerpunkt auf der Gates Foundation) und ihren Aktivitäten im Bildungsbereich, dass es für den Philanthropiesektor besser ist, über Bildung und z.B. die Rolle von Lehrenden zu sprechen, als über Armut, denn:

»[...] if the wealthy financiers were to admit that a substantial part of the problem was poverty and not bad teachers, the question would be why people like them are allowed to make so much when others have so little.« (McCoey 2016, S. 129$)^{90}$

90 Das Zitat im Kontext lautet wie folgt: „Strangely, the greater the debilitating toll poverty takes on the lives of America's schoolchildren, the more taboo disucssion of poverty have become. Pointing out the link between poverty and education is deemed an sexcuse seized on by tea- 
Damit drängt sich im Hinblick auf die hier im Mittelpunkt stehende außerschulische politische Jugendbildung in Deutschland verstärkt die Frage auf, wie das Aufgreifen progressiver Forderungen durch unternehmensnahe Stiftungen aus einer kritisch-emanzipatorischen politischen Bildungsperspektive $\mathrm{zu}$ bewerten ist: Inwiefern überschneiden sich die Ansätze mit den Anforderungen an eine kritisch-emanzipatorische politische Bildung, an welchen Stellen weichen sie davon $a b$ ? Dafür müsste eine genauere Analyse von Programmen vorliegen; dieser wird sich nachfolgend gewidmet.

\subsection{Das Beispiel Robert Bosch Stiftung und ihr Schwerpunkt politische Bildung für swenig interessierte Jugendlicher}

\subsection{1 Über die Stiftung}

Die Ausführungen in diesem Eingangskapitel dienen dazu, die Komplexität von Stiftungsstrukturen und die >Verwobenheit< von Stiftungen und Unternehmen an dem Beispiel Robert Bosch Stiftung zu veranschaulichen. Die RBS bietet sich in dieser Studie dafür an, da sie drei Modellprojekte zur politischen Bildung mit sbenachteiligen Jugendlichen pitel 4.3.3). ${ }^{91}$ Eine eingehendere Befassung mit Stiftungsstrukturen ist notwendig, wenn Stiftungsarbeit unter der Prämisse der >Universalisierung von Partikularinteressen< (vgl. Kapitel 3.1) betrachtet werden soll, da zunächst geklärt werden muss, welche Verbindungslinien zwischen Stiftungen und Unternehmen gezogen werden können.

Robert Bosch gründete 1886 die »Werkstätte für Feinmechanik und Elektrotechnik« (Robert Bosch Stiftung 2017a), aus der sich ein international tätiges Unternehmen, die heutige Robert Bosch $\mathrm{GmbH}$, entwickelte. Die nach ihm benannte Stiftung wurde von Robert Bosch gestiftet, die Gründung erfolgte durch seine Testamentsvollstrecker im Jahr 1964. Sie »formten« Boschs »Willen für die Zukunft in der sogenannten >Verfassung des Hauses Bosch'« (dies. 2017b). Damit ist eine

chers to exonerate their poor performance. [...] The topic of poverty is quietly ignored, just as politics or religion are ignored in polite company. Why is this? One answer is simple, if cynical. As a recent article in the New York Times suggests, if the wealthy financiers were to admit that a ssubstantial part of the problem was poverty and not bad teachers, the question would be why people like them are allowed to make so much when others have so little«.« (McGoey 2016, S. 129) McGoey zitiert hier den Artikel »Teachers Get Little Say in a Book About Them« von Michael Winerup (2011).

91 Die Umschreibung > wenig interessierte Jugendliche ‘ findet sich auf der Website der RBS bei der Vorstellung ihres Schwerpunkts »]ugend und Demokratie« (vgl. Robert Bosch Stiftung 2017u). Zu den einzelnen Zielgruppenbeschreibungen in den Modellprojekten: vgl. Kapitel 4.3.3. 
Struktur gemeint, die aus vier Bausteinen besteht: dem Unternehmen, der Industrietreuhand KG, der Familie Bosch und der Vermögensverwaltung Bosch GmbH die letztgenannte wurde 1969 in die Robert Bosch Stiftung GmbH umbenannt (vgl. ebd.). ${ }^{92}$ Die Stiftung hat bis heute ihren Sitz in Stuttgart, zudem - wie auch andere unternehmensnahe Stiftungen ${ }^{93}$ - eine »Repräsentanz« in Berlin-Mitte (dies. 2017c). In der Stiftung arbeiten aktuell rund 180 Mitarbeiter*innen (vgl. ebd.).

Die RBS genießt in der Öffentlichkeit eine hohe Reputation. ${ }^{94}$ Anders als bei der Bertelsmann Stiftung stellen ihre Verflechtungen mit dem Unternehmen oder ihre Politikberatungsaktivitäten selten Gegenstand fachlicher oder öffentlicher Diskussion dar, ${ }^{95}$ ihre Intransparenz - zum Beispiel die fehlende Veröffentlichung der Satzung - wird nicht problematisiert. ${ }^{96}$ Die hohe Reputation schlägt sich auch in dem gesellschaftlich breiten Spektrum an Partnern nieder, die sie für Kooperationen zu gewinnen vermag. Dieses Spektrum reicht z.B. im Bildungsbereich von wirtschafts- oder gewerkschaftsnahen Akteuren über NGOs bis hin zu staatlichen und supranationalen Institutionen.

Die Bosch Stiftung ist eine unternehmensverbundene Stiftung, ${ }^{97}$ da sie bis heute rund 92 Prozent Kapitalanteile am »Stammkapital der Robert Bosch GmbH « hält, das 1,2 Milliarden Euro beträgt (vgl. ebd.); sie ist somit Mehrheitseignerin der Robert Bosch GmbH. Zudem ist die RBS in der Form des Modells der »Doppelstiftung« organisiert, das wiederum das vorherrschende Modell bei den unternehmensnahen Stiftungen in Deutschland ist. ${ }^{98}$ Es lohnt sich daher, dieses Stiftungsmodell ein wenig ausführlicher zu betrachten.

Eine Doppelstiftung besteht aus einer gemein- und einer privatnützigen Stiftung (vgl. Bundesverband Deutscher Stiftungen 2013b, S. 36). Die RBS ist in diesem Modell die gemeinnützige Stiftung. Die gemeinnützige Stiftung im Modell

92 Zur Robert Bosch Stiftung gehören zudem »das Robert-Bosch-Krankenhaus, das Dr. MargareteFischer-Bosch Institut für Klinische Pharmakologie und das Institut für Ceschichte der Medizin der Robert Bosch Stiftung sowie die unselbständigen Stiftungen Hans-Walz-Stiftung, Otto und Edith Mühlschlegel Stiftung, DVA-Stiftung sowie Rochus und Beatrice Mummert-Stiftung«(Robert Bosch 2017c).

93 Z.B. die Bertelsmann Stiftung oder die BMW Stiftung Herbert Quandt.

94 Vgl. dazu u.a. die Reden von Angela Merkel (deutsche Bundeskanzlerin) oder Frank Walter Steinmeier (damaliger deutscher Bundesaußenminister) zum 50. Jubiläum (vgl. Robert Bosch Stiftung 2017d).

95 Holland-Letz diskutiert die Partikularinteressen der RBS (vgl. Holland-Letz 2015, S. 131ff.). Jüngst problematisierte die Zeitung taz, dass die Robert-Bosch-Stiftung Politiker*innen Reisen finanziert (vgl. Hinck 2017).

96 Jedoch veröffentlicht die RBS einen Jahresbericht, der auch die Ausgaben in einzelnen Bereichen und für Programmschwerpunkte nennt (vgl. Robert Bosch Stiftung 2017e).

97 Konkreter kann sie auch als >Unternehmensträgerstiftung im weiten Sinn〈oder auch als >Unternehmensbeteiligungsstiftung`gefasst werden (vgl. dazu die Ausführungen in Kapitel 4.1.1).

98 Schuler betont: »Nahezu sämtliche unternehmensnahe Stiftungen funktionieren nach diesem Prinzip.«(Schuler 2010, S. 294) 
der Doppelstiftung hält »die Mehrheit des Kapitals« am Unternehmen (Kögel/Berg 2011, S. 13), in diesem Falle also an der Bosch GmbH. Die privatnützige Stiftung wiederum hält die Mehrheit der Stimmrechte am Unternehmen (vgl. ebd.). ${ }^{99}$ Im Falle Bosch erfüllt diese Funktion jedoch keine privatnützige Stiftung, sondern - das ist nämlich auch möglich - eine »andere Gesellschaftsform[...] des Privatrechts« (als die der privatnützigen Stiftung) (ebd., S. 14): die sogenannte Industrietreuhand KG (vgl. Abb. 2). Sie übt die »Unternehmerische Gesellschafterfunktion aus « und verfügt über 93 Prozent der Stimmanteile in der Hauptversammlung des Unternehmens (vgl. Eulerich 2016, S. 323). Die restlichen Stimmanteile liegen bei der Bosch-Familie (vgl. ebd.). Bei der Industrietreuhand KG (und der Bosch-Familie) liegt somit die »unternehmerische Führung der Bosch Gruppe« (Kögel/Berg 2011, S. 15f.) und die Industrietreuhand KG fungiert als das höchste Kontrollorgan der Bosch GmbH. ${ }^{100}$ Sie kann somit als das »Zentrum der Macht « des Unternehmens betrachtet werden (Klesse 2012).

Abbildung 2: Die Robert Bosch Stiftung als Teil der Struktur der Robert Bosch Gruppe

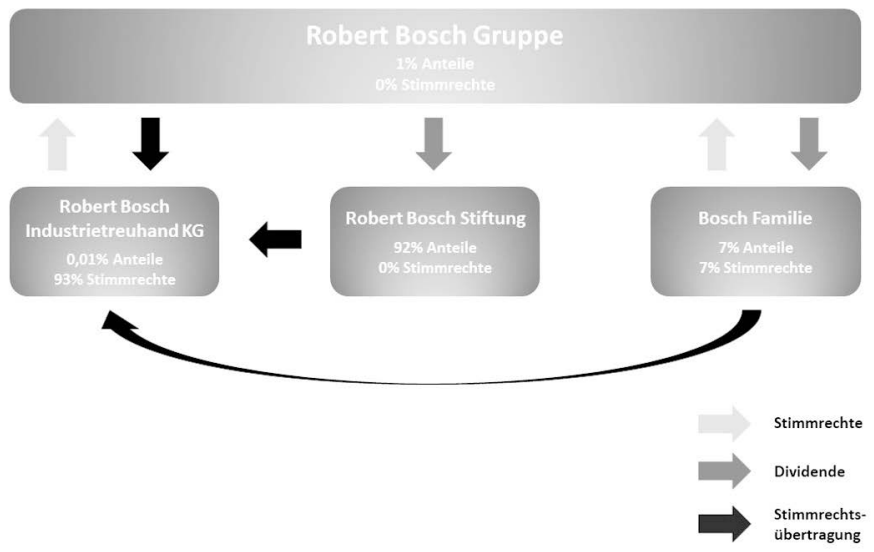

Quelle: Eulerich 2016, S. 323 (Nachbildung)

Die hier angedeutete Vielfalt für mögliche Institutionen, die die Stimmrechte am Unternehmen halten, kommt in der Realität auch zum Einsatz: Auch bei

99 Kögel/Berg schreiben, dass »die Mehrheit der Stimmrechte des Familienunternehmens [...] bei einer Familienstiftung « liegen (Kögel/Berg 2011, S. 13).

100 Eulerich/Welge befinden, dass die Industrietreuhand KG »als eine Art Aufsichtsrat [fungiert] « und bezeichnen sie weiterhin »als Kommanditgesellschaft firmierende[s] Kontrollgremium« (Eulerich/Welge 2011, S. 87f.). 
der Bertelsmann Stiftung ist das Gremium, das die Stimmrechte am Unternehmen hält, keine privatnützige Stiftung, aber auch keine Kommanditgesellschaft (wie beim Konstrukt Bosch), sondern die sogenannte »Bertelsmann Verwaltungsgesellschaft $\mathrm{mbH}$ « (Bertelsmann 2017c). Obwohl also die Bosch Stiftung und die Bertelsmann Stiftung Teil einer Doppelstiftung sind, unterscheiden sich die konkreten Stiftungskonstruktionen. Darin kann m.E. ein Grund gesehen werden, weshalb öffentlich bisher auch kaum diskutiert wird, dass die RBS - und viele andere gemeinnützige Stiftungen, die im Bildungsbereich aktiv sind - ganz ähnlich wie das umstrittene >Modell Bertelsmann Stiftung organisiert ist. ${ }^{101}$

Das Modell der Doppelstiftung ist so beliebt, weil es die Vorteile privat- und gemeinnütziger Stiftungsformen vereint: die Vorteile der "Stiftung bürgerlichen Rechts, etwa in Gestalt der Familienstiftung « und der steuerbegünstigten Stiftung (vgl. Kögel/Berg 2011, S. 16). So lägen die Vorteile der Familienstiftung beispielsweise in der »deutlich eingeschränkte[n] Fachaufsicht und (teilweise) Rechtsaufsicht durch die Stiftungsbehörden [...]«, in der "größtmögliche[n] Flexibilität in der Versorgung von Angehörigen « und in der Möglichkeit, »Betriebsvermögen schenkungs- und erbschaftssteuerfrei auf eine Familienstiftung « zu übertragen (ebd.). Der Vorteil der gemeinnützigen und deshalb steuerbegünstigten Stiftung liegt wiederum »insbesondere in ihrer steuerlichen Privilegierung« (ebd.). So können hier nicht nur Schenkungs- und Erbschaftsteuern bei der »Übertragung von Betriebs- und Privatvermögen auf die Stiftung « gespart werden, sondern auch die Stiftung selbst ist »vollständig von Ertragssteuern befreit « und »nicht verpflichtet, Körperschaft- oder Gewerbesteuer zu entrichten [...] « (ebd.). ${ }^{102}$ Obendrein ist schließlich »die Versorgung von Angehörigen [...] mit einem Drittel der Erträge der Stiftung steuerlich unschädlich möglich« (ebd.). Zur aktuellen »Verfassung des Hauses Bosch« (vgl. Abb. 2) gehören zwei Personengesellschaften, die die Familie Bosch besitzen und über die sie

»mittelbar an der Robert Bosch $\mathrm{GmbH}$ beteiligt [ist]. Über diese beiden Personengesellschaften steht der Familie Bosch ein Kapitalanteil von 7,36\% und ein Stimmrecht von 6,83\% zu. Die Satzung der Robert Bosch $\mathrm{CmbH}$ sieht zugunsten der Familie eine Vorzugsdividende vor, die laufende Auszahlungen an die Stifterfamilie sicherstellen soll.« (Kögel/Berg 2011, S. 16) ${ }^{103}$

$101 \mathrm{Vgl}$. dazu u.a. Schuler 2011, S. 224ff.; Holland-Letz 2015, S. $131 \mathrm{ff}$.

102 Kögel/Berg führen aus: „Die Stiftung ist nicht verpflichtet, Körperschaft- oder Cewerbesteuer zu entrichten, es sei denn, diese unterhält einen steuerpflichtigen wirtschaftlichen $\mathrm{Ce}$ schäftsbetrieb (z.B. Beteiligung an einem Cewerbebetrieb in der Rechtsform einer KC) [...] 《 (Kögel/Berg 2011, S. 16).

103 Eulerich/Welge verweisen 2011 darauf, dass »[d]ie Familiengesellschafter [...] rd. 5 Mio. Euro Dividende [erhalten]«(Eulerich/Welge 2011, S. 89). 
Die Versorgung der Nachkommen von Robert Bosch ist somit bis heute gesichert. Angesichts der umfänglichen Steuerersparungen und der z.T. eigennützigen Motive (wie die Versorgung von Familienmitgliedern) ist es einerseits wenig verwunderlich, dass die »Unternehmensverfassung« der RBS zum »Vorbild für viele unternehmensverbundene Stiftungen in den letzten 50 Jahren« wurde (ebd., S. 13). Andererseits überrascht es aber auch nicht, dass das Modell der Doppelstiftung in Fachkreisen umstritten ist: Zum Teil wird in ihm sogar ein "Gestaltungsmissbrauch « gesehen (ebd., S. 17). Wenig überraschend ist weiterhin, dass Rainer Kögel und Dieter Berg in ihrem Fachartikel zur »Unternehmensverfassung des Hauses Bosch als Grundmodell der Doppelstiftung« befinden, dass die Kritik am Modell der Doppelstiftung »nicht zu überzeugen [vermag]« (ebd.). Denn Berg war, als er den Text mitverfasste, Geschäftsführer der RBS. Berg und Kögel argumentieren, dass durch die »Modernisierung des Stiftungsrechts aus dem Jahre 2002« der »Gesetzgeber klargestellt« habe, »dass unternehmensverbundene Stiftungen grundsätzlich zulässig sind und keinen Missbrauch der Rechtsform Stiftung darstellen." (Ebd.) ${ }^{104}$ Das stimmt, ändert jedoch nichts daran, dass das Stiftungsmodell als politisch umstritten gelten muss und z.B. in den USA Stiftungen nicht mehr als 20 Prozent Anteile an Unternehmen halten dürfen (vgl. Kapitel 4.1.1).

Die Robert Bosch Stiftung ist somit Teil einer Unternehmensverfassung, in der Steuervermeidung und Versorgung der Nachkommen eine wichtige Rolle spielen. Dies sagt noch nichts über den Charakter der konkreten Stiftungsarbeit aus, lässt aber die eigennützigen Motive, die über das Stiftungsmodell realisiert werden können, erkennen und sollte m.E. somit zu einer grundsätzlich differenzierteren Beurteilung der Gemeinwohlorientierung von Stiftungen wie der RBS und ihrer wohltätigen Arbeit führen.

Vonseiten der Robert Bosch Stiftung wird gern die strukturelle Trennung vom Unternehmen hervorgehoben. So antwortet die ehemalige RBS-Geschäftsführerin Ingrid Hamm auf die Interviewfrage einer Wirtschaftszeitung, wie stark die RBS "mit dem Unternehmen vernetzt « sei, dass "ökonomische und philanthropische Ziele [...] getrennt und eigenständig verfolgt [werden]« (Hamm 2009). Die Ziele »Fortbestand und Weiterentwicklung des Unternehmens« einerseits und »Förderung gemeinnütziger Ziele« andererseits würden

»in unabhängigen Organisationen verwirklicht. Das macht nicht nur rechtlich und ethisch, sondern auch praktisch Sinn. Das Unternehmen sollte von Persönlichkeiten geführt werden, die etwas davon verstehen. Dasselbe gilt umgekehrt für die Stiftung.« (Ebd.)

104 Es findet sich auch der Verweis, dass erbschaftssteuerliche Nachteile bei unternehmensverbundenen Stiftungen durch das seit 2009 gültige Erbschaftssteuerrecht deutlich gemindert seien (vgl. Kögel/Berg 2011, S. 17). 
Anhand dieses Statements von Hamm könnte man davon ausgehen, dass sich in den Leitungsgremien von Unternehmen und Stiftung unterschiedliche Personen befinden - schließlich handelt es sich nach der Aussage von Hamm um sunabhängige Organisationen<. Doch es besteht eine

»sehr enge personelle Verflechtung zwischen der Stiftung und den Lenkungsorganen des Unternehmens. Viele Leute aus dem Stiftungsvorstand oder dem Beirat haben auch Lenkungs- und Überwachungsaufgaben im Unternehmen.« (Eulerich; zit.n.: Bundesverband Deutscher Stiftungen 2013b, S. 40) ${ }^{105}$

Eine wirkliche Trennung der Strukturen gibt es also nicht - genau genommen wirkt es nur so. Denn die personellen Verflechtungen verbinden die formal getrennten Strukturen von Unternehmen und Stiftung. Aktuell sind drei Mitglieder aus der Gesellschafterversammlung (das zugleich als Kuratorium fungiert) der RBS auch in der Industrietreuhand KG (Christof Bosch und Siegfried Dais) bzw. im Aufsichtsrat der Bosch GmbH (Christof Bosch, Matthias Madelung) vertreten (vgl. Robert Bosch Stiftung 2017g; Bosch 2017b). Christof Bosch, der neben seinem Sitz in der Industrietreuhand KG und im Aufsichtsrat der Bosch GmbH auch die Funktion des Sprechers der Familie Bosch hat, führt seit März 2017 den Vorsitz des RBS-Kuratoriums (vgl. Robert Bosch Stiftung 2017f). ${ }^{106}$

Für Außenstehende sind diese Personalunionen nicht leicht ersichtlich, sie müssen recherchiert werden, denn die Funktionen der Mitglieder der Gesellschafterversammlung (respektive Kuratorium) werden auf der RBS-Website nicht genannt. Wer sich nicht näher mit der Verfassung der RBS (Stichwort >Doppelstiftungく, die unterschiedlich konstruiert sein kann) oder des Unternehmens Bosch befasst hat, würde zudem vielleicht eher nach Personalunionen im Unternehmensvorstand suchen - und nicht in der Industrietreuhand KG, dem eigentlichen Machtzentrum der Bosch-Gruppe. Dies kann z.B. auch an der unternehmensverbundenen Freudenberg Stiftung ${ }^{107}$ verdeutlicht werden: Die Mitglieder der Gesellschafterversammlung der Stiftung werden ebenfalls nicht mit Funktion auf der Stiftungs-Website genannt. Damit bleibt zunächst verborgen, dass ein Gesellschafter der Stiftung, Mohsen Sohi, zugleich Sprecher des Vorstands der Freudenberg SE ist (vgl. Freudenberg SE 2017).

Diese personellen Verflechtungen sind m.E. umso relevanter, als dass die RBS über kein Kontrollgremium verfügt (vgl. zu Stiftungsstrukturen Kapitel 4.1.2). Sie gibt zwar insgesamt drei Stiftungsorgane an: die Gesellschafterversammlung, das

105 Eulerich trifft diese Aussage für die Unternehmen Bosch und Thyssen Krupp in einem Interview mit dem BDS (vgl. Eulerich; zit.n.: Bundesverband Deutscher Stiftungen 2013b, S. 40).

106 Diese Personalunion wird auch von Eulerich/Welge festgestellt (vgl. Eulerich/Welge 2011,

S. 89), aber nicht dahingehend problematisiert, dass sie nicht zum Postulat der getrennten Strukturen passt.

107 Zur Arbeit der Freudenberg Stiftung auf dem Feld der politischen Bildung: vgl. Kapitel 4.2.2. 
Kuratorium und die Geschäftsführung (vgl. Robert Bosch Stiftung 2017c), jedoch sind die ersten beiden Organe mit den gleichen Personen besetzt und letzteres stellt kein Kontrollorgan dar. Das Kuratorium (in dem zwei Personen aus der Industrietreuhand KG vertreten sind) hat wesentlichen Einfluss auf die Stiftungsarbeit, denn es »[entscheidet] [ü]ber die gemeinnützigen Aktivitäten und die Mittelverwendung der Stiftung (ebd.); »nach dem Willen von Robert Bosch« ist es mit »Mitglieder[n] der Familie, Unternehmer[n] und Persönlichkeiten des öffentlichen Lebens« (ebd.) besetzt.

Das Unternehmen Bosch GmbH kommuniziert die gemeinnützigen Tätigkeiten der RBS im Rahmen seiner CSR-Tätigkeiten (unter dem Label »Nachhaltigkeit«) (vgl. Bosch 2017a). Dies mag in Zeiten der steigenden Bedeutung von CSR aus Unternehmenssicht sinnvoll erscheinen, widerspricht gleichzeitig aber der viel beschworenen >Trennung von unternehmerischen und philanthropischen Zielen $<{ }^{108}$ Inwieweit die RBS inhaltliche Überschneidungen mit den Geschäftsfeldern der Bosch $\mathrm{GmbH}$ aufweist (wie z.B. die Bertelsmann Stiftung mit dem BertelsmannKonzern), ${ }^{109}$ ist bislang nicht systematisch untersucht. Mittelbare Bezüge der Stiftungsaktivitäten zu Geschäftsinteressen sind jedoch erkennbar. So befindet Jennifer Andres in ihrer Betrachtung der RBS:

»Auffällig ist, dass die Stiftung mitunter von marktwirtschaftlichen Interessen geleitete Projekte initiiert. Die Einrichtung eines deutsch-chinesischen, deutsch-japanischen und deutsch-indischen Schwerpunktes auf dem Gebiet der Völkerverständigung im Jahr 2006 mit der Förderung von Projekten wie zum Beispiel der >Wissenschaftsbrücke China ist unter dem Blickwinkel des starken wirtschaftlichen Aufschwungs in China und den dadurch hervorgerufenen ansteigenden Investitionen von Unternehmen in Richtung Asien sicherlich kein Zufall.« (Andres 2008, S. 17)

Diese Ausrichtung der RBS erklärt sich Andres damit, »dass die Stiftung in den letzten Jahren insgesamt sehr viel näher an das Unternehmen Bosch herangerückt ist « (Andres 2008, S. 17). Andres stellt einen Zusammenhang zwischen den Personalunionen (in Stiftung und Unternehmen) und der Stiftungsarbeit her:

»Angesichts dieser Verflechtungen und der Tatsache, dass das Kuratorium der Stiftung über Förderungspolitik, Entwicklung von Programmen sowie die Cenehmigung von Projekten oder Zuwendungen entscheidet, stellt sich die Frage, inwieweit die Trennung von ökonomischen und philanthropischen Cesichts-

108 Auch Anheier et al. stellen für Unternehmensträgerstiftungen, die »Allein- oder Mit-Trägerin eines Unternehmens « sind, fest, dass die Stiftungen »weit in die Governance des Unternehmens hineinwirken können - und umgekehrt«, mithin also auch ein Einfluss des Unternehmens auf die Stiftungsgovernance gegeben ist (Anheier et al. 2017a, S. 15).

109 Vgl. dazu u.a. Füller 2015. 
punkten tatsächlich aufrechterhalten werden kann. Diese Frage dürfte durch den, auf die Entwicklung des Unternehmens Bosch wirkenden Clobalisierungsdruck, noch größere Bedeutung erlangen, da das Unternehmen geneigt sein könnte, für seine weltweiten Aktivitäten von der Stiftung noch weitreichendere Unterstützung zu erwarten.« (Ebd., S. 18)

Eulerich/Welge stellen zudem mit Blick auf das unternehmerische und stifterische Engagement fest, dass es einen »Zielkonflikt im Erreichen beider Ziele« gibt (Eulerich/Welge 2011, S. 89). Sie führen aus, dass das Erzielen einer hohen Rendite an erster Stelle steht:

»Trotz des Umstands, dass der Bosch-Konzern über eine Eigenkapitalquote von $46 \%$ verfügt und dass die Familie den größten Teil des Cewinns vor Steuern von 3,2 Mrd. Euro im Vorjahr im Konzern belässt, achten Familie und Stiftung dennoch auf die Erzielung einer maximalen Rendite. Verluste hingegen finden selbst mit dem Hinweis auf die Übernahme gesellschaftlicher Verantwortung und Rücksichtnahme keinen positiven Zuspruch und sind nicht akzeptiert.« (Ebd., S. 88)

Darin zeigt sich, dass die »Maxime«, die schon bei der Gründung im Jahr 1964 galt, immer noch aktuell ist: ")Erst das Unternehmen, dann die Stiftung" (ebd., S. 89). ${ }^{110}$ Das Unternehmen lässt der Stiftung eine Dividende zugehen - umso mehr sie der Stiftung gibt, umso weniger bleibt für das Unternehmen. Eulerich/Welge geben 2011 an, dass das Unternehmen »jährlich rd. 2,5 \% des Nettogewinns an die Stiftung aus[schüttet]« (ebd.); die Dividende betrug für das Jahr 2016 rund 126,8 Millionen Euro (vgl. Robert Bosch Stiftung 2017e, S. 57). ${ }^{111}$

Stiftungen ermöglichen ihren Gründer*innen, ein gutes Image über sich selbst als Unternehmer*innen zu verbreiten. Die Robert Bosch Stiftung hebt z.B. hervor, dass Robert Bosch »als Erster in Deutschland den 8-Stunden-Tag für seine Mitarbeiter « eingeführt habe und »Bildungsmöglichkeiten für die Bürger, von der Volkshochschule bis zur Begabtenförderung [förderte]« (dies. 2017n). Die »Ausund Weiterbildung seiner Mitarbeiter« sei ihm »ein besonderes Anliegen« gewesen

110 Das Stiftungsmodell habe »eine kontinuierliche Expansion der Bosch Cruppe ermöglicht« (Kögel/Berg 2011, S. 13).

111 Darüber hinaus gibt es »[w]eitere Zuflüsse aus Spenden, unselbstständigen Stiftungen und Zinsen«, wobei diese »nachrangig« seien; auch gehe man oft »für ein bestimmtes Projekt eine Partnerschaft ein [...] ( Hamm 2009). Holland-Letz befindet auf Basis der Ausschüttung von 176 Millionen, also 7 Prozent des Nettogewinns der Bosch GmbH 2014 (der bei 2,6 Mrd. lag), dass die RBS damit im Vergleich zur Bertelsmann Stiftung oder zur Possehl-Stiftung wenig ausschütte (vgl. Holland-Letz 2015, S. 131f.). M.E. sind Einschätzungen solcher Art mit Vorsicht zu genießen, da jede Stiftung unterschiedliche Berechnungsgrundlagen zugrunde legt. Holland-Letz gibt hiermit dennoch einen weiteren wichtigen Hinweis für den Bedarf an systematisch-vergleichenden Untersuchungen. 
(ebd.). Während die RBS den Unternehmer Bosch für seine »vorbildliche[n] soziale[n] Leistungen« (ebd.) lobt, stellen Johannes Bähr und Paul Erker in ihrer historischen Untersuchung zum Unternehmen Bosch heraus, dass es Robert Bosch »bei der Einführung des Achtstundentags keineswegs nur um eine soziale Pioniertat [...]« ging, sondern die »kürzere Arbeitszeit zu Produktivitätssteigerungen führen« sollte (Bähr/Erker 2013, S. 65).

Die Widersprüchlichkeit, die sich im sozialen Engagement von Unternehmer*innen finden lässt, kann auch anhand eines Statements der ehemaligen RBS-Geschäftsführerin Hamm diskutiert werden. Auf die Frage, wie Bosch mit der Krise in den Nullerjahren umgegangen wäre, antwortet sie: »Es wäre ihm sehr wichtig gewesen, die Arbeitnehmer zu halten, denn er wusste, dass seine Mitarbeiter sein Kapital sind.« (Hamm 2009) Einerseits können gut ausgebildete Arbeitnehmer"innen den Profit eines Unternehmens steigern, andererseits ist die Rücksichtnahme auf die Interessen von Arbeitnehmer*innen offensichtlich besser als Rücksichtslosigkeit. Robert Bosch schien sich immerhin nicht im extremen Widerspruch $\mathrm{zu}$ seinem philanthropischen Handeln $\mathrm{zu}$ befinden, wie z.B. der US-Unternehmer Andrew Carnegie. Dieser gründete nicht nur zahlreiche große (und bis heute einflussreiche) Stiftungen, sondern muss auch mit einer der blutigsten Arbeiteraufstände der US-Geschichte in Zusammenhang gebracht werden (vgl. McGoey 2016, S. 10). ${ }^{112} \mathrm{Zu}$ dieser Ambivalenz philanthropischen Handelns gibt es in Deutschland bisher kaum Untersuchungen. ${ }^{113}$ Dies führt dazu, dass das soziale Image von Unternehmer*innen über ihre Stiftungen ohne kritische Prüfung öffentlich gepflegt werden kann.

\subsubsection{Strategie im Schwerpunkt »Jugend und Demokratie»}

Aktuell ist die RBS in fünf Fördergebieten aktiv: Gesundheit, Wissenschaft, Gesellschaft, Bildung und Völkerverständigung (vgl. Robert Bosch Stiftung 2017h). In den drei letztgenannten Themenbereichen finden sich auch Programme der politischen Bildung wieder. Bereits 1998 »wurde beschlossen, dass die Förderung von Ehrenamt und bürgerschaftlichen Engagements, ein profilgebendes Element sowie Querschnittaufgabe in der zukünftigen Arbeit der Stiftung sein soll« (Andres 2008, S. 12). ${ }^{114}$

112 Vgl. ausführlich zu den Geschehnissen: McGoey 2016, S. $10 f$.

113 Auf die Geschichte der Robert Bosch Stiftung kann an dieser Stelle nicht vertieft eingegangen werden. Der Rückgriff auf eine unabhängige, wissenschaftliche Aufarbeitung ihrer historischen Ursprünge, Entwicklung und aktuellen Ausrichtung wäre hilfreich, jedoch gibt es diese nicht. Es existiert nur eine von der RBS herausgegebene Chronik (vgl. Robert Bosch Stiftung 2000).

114 Andres bezieht sich hier auf die Chronik der Robert Bosch Stiftung (vgl. Robert Bosch Stiftung 2000, S. 112). 
Wie schon erwähnt, lassen sich politische Bildungsprogramme für Jugendliche und junge Erwachsene aus unterschiedlichen Bildungskontexten finden (für Haupt-/Realschüler*innen, Azubis/Berufsschüler*innen, Gymnasiast"innen oder Studierende) sowie zahlreiche Programme, die sich an Multiplikator*innen (wie Journalist*innen, Lehrer*innen oder Politiker*innen) richten (vgl. Kapitel 4.2.2). Wie nachfolgend ausgeführt wird, legt die RBS einen ihrer Schwerpunkte in der politischen Bildung auf sogenannte spolitikferne Zielgruppen` (vgl. Schuster 2012a).

Nicht nur in der politischen Bildung setzt sich die RBS mit >bildungsbenachteiligten Jugendlichen auseinander, sie engagiert sich auch im Bereich Bildungspolitik oftmals in Bezug auf diese: Sie vergibt den Deutschen Schulpreis, für dessen Vergabe der »Umgang mit Vielfalt« ein Kriterium ist (Robert Bosch Stiftung 2017k). Außerdem fördert die RBS das Programm »School Turnaround « zur Unterstützung von »Schulen in kritischer Lage« (dies. 2017i; Holland-Letz 2015, S. 82ff.). Investitionen in Bildung verortet Hamm, die bis 2015 als RBS-Geschäftsführerin eingesetzt war, allgemein im Kontext wirtschaftlicher Erfordernisse:

»Wir halten es für einen Fehler, dass in Deutschland Bildungsausgaben so wenig als Zukunftsinvestitionen gesehen werden. Wir sind unter OECD-Durchschnitt, und dafür gibt es in einem Land, das nur über Humankapital verfügt, keine Rechtfertigung.« (Hamm 2009)

In dem Interview aus dem Jahr 2009 führt sie weiter aus, dass Bildung nicht nur für die »Exportweltmacht« Deutschland wichtig ist, sondern verallgemeinert dieses wirtschaftliche Interesse als eines, das für jede/n Einzelne/n als >Versicherung gegen Arbeitslosigkeit< wichtig sei:

»[...] wir haben uns viele Gedanken gemacht, welche Konsequenzen die Krise für die Cesellschaft hat. Wir sind zum Schluss gekommen, dass sowohl für das Individuum als auch für unsere Cesellschaft der Weg aus der Krise nur über Bildung führt. Bildung ist die einzige Versicherung, die man persönlich wie auch als Innovationsstandort und Exportweltmacht hat. Wir müssen unsere Innovationskraft und Bildungsqualität in der Welt bewahren, wenn wir nach der Krise wirtschaftlich gestärkt sein sollen. Für den Einzelnen heißt das, Arbeitslosigkeit kann jeden treffen, aber langfristig bleibt derjenige am längsten arbeitslos, der am wenigsten gebildet ist.« (Ebd.)

Wie nachfolgend gezeigt wird, schlägt sich dieser Fokus auf die Stärkung des Individuums auch in den politischen Jugendbildungsprogrammen mit sbenachteiligten Jugendlichen nieder (vgl. Kapitel 4.3.3.4). Die Robert Bosch Stiftung stößt in diesem Bereich in die (im Kapitel 2.2.2) beschriebene »Lücke« (Kohl/Seibring 2012 b, S. 8) an innovativer Formatentwicklung in der politischen Jugendbildung und hat in den letzten Jahren drei Modellprojekte gemeinsam mit unterschiedli- 
chen Partnern gefördert (vgl. Schuster 2012, S. 117-129). Zu dieser Schwerpunktsetzung, die die RBS in der politischen Bildung verfolgt, scheint die Einschätzung von Adloff/Strachwitz zu passen, die in der RBS »ein Beispiel für staatsunabhängiges Handeln« sehen, »das sich am Gemeinwohl und explizit am Ziel orientiert, Alternativen zum Mainstream über Innovation bereitzustellen« (Adloff/Strachwitz 2004, S. 115; zit.n.: Andres 2008, S. 13). Die Gemeinwohlorientierung von Stiftungen, dies wurde bereits ausführlich dargelegt, ist dabei stets als "private Vision der Gemeinwohlorientierung « (Adloff 2010, S. 415) zu verstehen. Wie nun diese sprivate Vision von Gemeinwohlorientierung der RBS aussieht, wird in ihrer internen Strategie zum Programmschwerpunkt »Jugend und Demokratie« deutlich (vgl. Robert Bosch Stiftung 2014). ${ }^{115}$

In der Strategie begründet die RBS ihr Engagement in der politischen Bildung zunächst mit Bezug auf Robert Bosch: Er habe schon zu Lebzeiten die 1919 gegründete Deutsche Hochschule für Politik in Berlin gefördert; ${ }^{116}$ außerdem sei es ihm »bei Bildungsprozessen gerade nicht darum« gegangen,

»die Menschen vollzutrichtern mit allen möglichen Dingen, die mechanisch gelernt werden, um ein Examen bestehen zu können<, sondern darum, seinen eigenen Standpunkt, eine gefestigte Anschauung über Ereignisse aller Art [zu] bekommen.«(Ebd.) $)^{117}$

Weiterhin wird in der Strategie ein Bezug zu den Satzungszielen der RBS hergestellt:

»Entsprechend ihren Satzungszielen fördert die Robert Bosch Stiftung die gleichberechtigte Teilhabe am gesellschaftlichen Leben unter Respektierung von Vielfalt, eine demokratische, tolerante Bürgergesellschaft sowie gleiche Bildungschancen für alle Bürger. Wir wollen im Besonderen junge Menschen ermutigen, Verantwortung in der Gesellschaft zu übernehmen und sich für ihre Interessen einzusetzen.« (Ebd.)

Die »aktuellen Diskussionen über eine verstärkt in unserem Land zu beobachtende Politikverdrossenheit « waren für die RBS 2009 der Grund, einen Schwerpunkt »Politische Bildung« (bzw. »Jugend und Demokratie«) einzurichten (ebd.). Dabei geht sie davon aus, dass politische Bildung das demokratische Bewusstsein schärfe und qualifiziere, da sie Einblick in komplexe Zusammenhänge in Politik, Wirtschaft, Gesellschaft und Medien gäbe, »zur Übernahme von Verantwortung/Partizipation« ermutige, die Diskussionskultur stärke und »Kompetenzen für eine Kultur

115 Die RBS hat die (nicht veröffentlichte) Strategie freundlicherweise zur Verfügung gestellt.

116 Aus der Deutschen Hochschule für Politik ging das heute an der Freien Universität Berlin angesiedelte Otto-Suhr-Institut für Politikwissenschaft hervor (vgl. Freie Universität Berlin 2017). 117 In dem Ausschnitt wird aus der Bosch-Biographie von Heuss zitiert (vgl. Heuss 2008, S. 553). 
der fairen Auseinandersetzung « vermittle (ebd.). Dies sind Aussagen, die sicher viele Akteure der politischen Bildung unterstützen würden. Aus der Perspektive kritisch-emanzipatorischer politischer Bildung fällt auf, dass in der Strategie die Auseinandersetzung mit Macht- und Herrschaftsverhältnissen nicht eingefordert wird. Politische und gesellschaftliche Beschränkungen, die verhindern, dass sich jemand Gehör verschaffen kann, werden z.B. nicht genannt.

In der Strategie wird weiter ausgeführt, dass "geringe Wahlbeteiligung « und das schlechte Image von Politiker*innen auf das geringe Interesse von Jugendlichen »für (Partei-)Politik« verwiesen:

»Es scheint für sie eine Distanz zwischen der eigenen Lebenswelt und dem politischen System zu klaffen. Viele Jugendliche haben das Cefühl, auf dieses System keinen Einfluss zu haben, und verweigern ihre Beteiligung.« (Ebd.)

Direkt im Anschluss an diese Feststellung wird darauf hingewiesen, dass »[d]ie Notwendigkeit für neue Angebote der politischen Bildung für sogenannte politikferne Zielgruppen [...] außer Frage [steht]«, da

»[z]ahlreiche Studien belegen, dass bildungsferne Jugendliche sich seltener engagieren, seltener partizipieren und ein deutlich geringeres Interesse an Politik aufweisen als jugendliche Bildungsgewinner. Dies gilt z.B. auch für eine große Gruppe der Jugendlichen mit Migrationshintergrund. Cleichzeitig werden diese Jugendlichen kaum zielgruppengerecht von Angeboten politischer Bildung erreicht.«(Ebd.)

In der Strategie wird auch die bildungspolitische Lage der politischen Bildung benannt und darauf hingewiesen, dass »die Bundes- und die Landeszentralen für politische Bildung in den letzten Jahren massive Kürzungen erfahren« haben und das,

»[o]bwohl das System der staatlichen politischen Bildung in Deutschland international als Vorzeigemodell gilt und die Wichtigkeit politischer Bildung v.a. im Nachgang von politisch motivierten Straftaten immer wieder besonders betont wird [...].«(Ebd.)

Die RBS möchte deshalb mit ihrem »Schwerpunkt Jugend und Demokratie [...] den Stellenwert nachhaltiger politischer Bildung stärken und zugleich Mut für unkonventionelle Zugänge machen« (ebd.). Man wolle hier jedoch keine »Lückenfinanzierung« übernehmen, sondern »komplementär [wirken]«; »[a]ls private Stiftung« sei man »in der privilegierten Lage, neue Wege in der politischen Bildung zu gehen und schnell auf wissenschaftliche Befunde zu reagieren « (ebd.). Die Projekte im Schwerpunkt »ugend und Demokratie« würden »über Alleinstellungsmerkmale« verfügen, »die sie auch für die Fachdisziplin der politischen Bildung zu einem in- 
teressanten Referenzpunkt machen« (ebd.). Denn wie die RBS - übereinstimmend mit empirischen Befunden ${ }^{118}$ - feststellt, lassen sich kaum Akteure finden, die

»neue Ansätze praktisch erproben und dabei einen langen Atem beweisen. Hier können wir durch innovative Modellvorhaben, durch Studien und Evaluationen eine sinnvolle Unterstützung für die Praxis und den Fachdiskurs innerhalb der politischen Bildung leisten und wichtige Impulse setzen.« (Ebd.)

Letzteres wird z.B. dadurch realisiert, dass Erkenntnisse aus Modellprojekten und Begleitevaluationen in einschlägigen Fachjournalen und Sammelbänden (vgl. Schuster 2012a, b) und auf Fachtagungen vorgestellt werden. ${ }^{119}$ Damit finden die Zielstellungen der RBS in der politischen Bildung weite Verbreitung in der politischen Bildungsszene. Als Ziele der »Projekte[...] im Schwerpunkt »Jugend und Demokratie« werden genannt:

- "politikferne Jugendliche als Zielgruppe politischer Bildung stärker in den Fokus der Fachöffentlichkeit und der Politik rücken;

- Jugendliche unterstützen, urteilend und handelnd Einfluss auf ihre Angelegenheiten in der Gesellschaft zu nehmen;

- die Entwicklung einer demokratischen Identität fördern und demokratische Handlungsfähigkeit und -kompetenz vermitteln;

- das Verständnis für die Grundlagen und Zusammenhänge unseres Gemeinwesens stärken und eine Haltung des aktiven und mündigen Einstehens und Handelns für demokratische Werte fördern;

- das Interesse an Politik und die politische Partizipation von Jugendlichen stärken« (Robert Bosch Stiftung 2014).

Ein wichtiger Punkt sei - genau wie im vorherrschenden Diskurs in der politischen Bildung (vgl. Kapitel 2.2.2) - wie man Jugendliche (in dem Schwerpunkt Jugend und Demokratie`) »zielgruppenspezifisch« erreicht: Die RBS konzentriere sich auf

»politikferne Zielgruppen, die zu erreichen und zu motivieren eine besondere Herausforderung darstellt. Es geht uns dabei weniger um die Vermittlung von Fachwissen und klassischer Institutionenkunde, sondern um die Veränderung von Haltungen und die Stärkung von Kompetenzen für demokratisches Han-

118 Vgl. dazu die (zweite) Empfehlung von Becker für die politische Bildungsforschung: Becker 2011a, S. 162.

119 So stellte z.B. Anja Besand die Ergebnisse aus der Evaluation von »DU HAST DIE MACHT « auf der Tagung »Praxis und Wissenschaft im Dialog. Ansprache - Methoden - Konzepte in der politischen Bildung mit bildungsbenachteiligten Jugendlichen« 2012 in Cöttingen vor (vgl. Bundeszentrale für politische Bildung 2017d). 
deln und um die Beschäftigung mit grundlegenden Fragen und Problemen des gesamtgesellschaftlichen Zusammenlebens.« (Robert Bosch Stiftung 2014)

Um die Jugendlichen »in ihren Lebenswirklichkeiten« zu erreichen, gehe man in die Schule, ins Fußballstadion oder in die neuen Medien: "In unterschiedlichen Projekten versuchen wir adäquate Zugänge zu jungen Menschen zu schaffen und sie für Politik und politische Beteiligung zu sensibilisieren.« (Ebd.)

Wie noch ausführlicher gezeigt wird, beschreitet die RBS hier mutig neue Wege und fördert experimentelle neue Ansätze, die sich so bisher noch nicht im politischen Bildungsbereich finden lassen. Im Vergleich $\mathrm{zu}$ anderen Stiftungen, die z.T. ausschließlich Elitenförderung betreiben (vgl. Kapitel 4.2.2), hebt sie sich damit positiv ab. Legt man das eingangs begründete Verständnis zugrunde, dass die liberale Demokratie als eine eingeschränkte betrachtet werden kann (vgl. Kapitel 2.2.1), deutet sich jedoch in den Ausführungen in der Strategie auch schon an, dass die Thematisierung und Analyse sozialer und politischer Ausschlüsse nicht zu den (prioritären) Zielen gehören. Eine »kritische[...] Auseinandersetzung mit gesellschaftlichen Ungleichheitsverhältnissen«, wie sie Scherr für die Jugendbildung einfordert (Scherr 2011, S. 52; vgl. auch Kapitel 2.2.3), wird hier nicht als Gegenstand für die politische Bildung genannt. Wie auch schon in der Analyse des Forschungstands zur politischen Bildung mit >benachteiligten Jugendlichen deutlich wurde, wird dies auch im breiteren politischen Bildungsdiskurs kaum thematisiert. Dieser Eindruck lässt sich bei der näheren Betrachtung der RBSModellprojekte und den dazu vorliegenden Evaluationen erhärten.

\subsubsection{Innovative Modellprojekte}

Nachfolgend werden die Modellprojekte der RBS näher betrachtet. In diesen kooperiert die RBS mit unterschiedlichen Partnern und entwickelt neue Formate, in deren Rahmen neue Multiplikator*innen für die politische Bildung ausgebildet, neue Lernorte und -medien erschlossen und starke Anerkennungspraxen sichtbar werden, z.B. dadurch, dass man nach Themeninteressen von Jugendlichen fragt. Auch wird ersichtlich, dass die Programme breite öffentliche Anerkennung erfahren, legt man dafür Schirmherrschaften und Auszeichnungen als Kriterien zugrunde.

Alle drei Modellprojekte der RBS wurden zudem im Rahmen formativer Evaluationen begleitend untersucht und Zwischenergebnisse in die Praxis zurückgespielt. Dies ist mit hohen Kosten verbunden und offensichtlich nur für ressourcenstarke Akteure machbar (wie es schließlich auch die bpb als ein Kooperationspartner der RBS ist). Obwohl es genau einer solchen Forschung zur Weiterentwicklung außer- 
schulischer politischer Bildung bedarf, hat eben jene großen Seltenheitswert. ${ }^{120}$ Denn die »Evaluationspraxis« in der politischen Bildungsforschung

»dient [...] meist der nachträglichen Absicherung von Förderentscheidungen, anstatt dass sie sauf Qualitätskontrolle, Beratung und Weiterbildung der Mitarbeiter und Mitarbeiterinnen setzt, und [...] Projekte als Fundus neuer Erfahrungen, als Feldarbeit mit einem sehr veränderlichen Objekt nutzt.« (Becker 2011a, S. 20$)^{121}$

Besonders in den Evaluationen zu den Programmen »DU HAST DIE MACHT«(DHDM) und »Dialog macht Schule«, die unter der Federführung der Fachdidaktikerin Anja Besand durchgeführt wurden, wird m.E. jedoch eine prozessbegleitende Forschung sichtbar, an der es in der »desolat[en]« empirischen außerschulischen politischen Bildungsforschung bislang oftmals fehlt (Becker 2011a, S. 161).

Anhand einer Inhaltsanalyse soll nachfolgend in einem ersten Schritt (Kapitel 4.3.3.1 bis 4.3.3.3) aufgezeigt werden, welche innovativen Wege beschritten werden, um Jugendliche zu erreichen, aber auch um die Programme zu evaluieren. Zudem soll anhand der Evaluationsergebnisse dargestellt werden, aus welchen Gründen die Formate bei den Jugendlichen gut ankommen. In einem zweiten Schritt (Kapitel 4.3.3.4) werden die in Programmbeschreibungen und Evaluationen formulierten Bildungsziele herausgearbeitet und auch der Frage nachgegangen, ob und inwiefern in diesen Programmen Unternehmensinteressen zur Geltung kommen. Als Quellen dienen die Websites der RBS und ihrer jeweiligen Programmpartner, Programmbroschüren und Programmevaluationen sowie ein Gedächtnisprotokoll, das nach der Teilnahme an einem Workshop des Programms »Lernort Stadion« angefertigt wurde. ${ }^{122}$

120 Becker konstatiert: »Forschung, die sich direkt und zusammen mit betroffenen Trägern der Praxis politischer Bildung widmet, ist selten.«(Becker 2011a, S. 19) Möller führt dazu aus, dass »[a]ufgrund mangelnder Kontakte zwischen Forschung und Praxis und insbesondere fehlender Kontinuität von Austausch und Kooperation [...] die Praxis allenfalls punktuell neuere Erkenntnisse wahrnehmen [kann]. [...] Umgekehrt fallen Beiträge aus der Sphäre der Wissenschaften aus der Sicht von Praktiker/innen (zu) selten anwendungsorientiert aus, kümmern sich Hochschulen und Forschungsinstitute zu wenig um relevante Praxisfragestellungen und entsteht so im Gegenzug zur wahrgenommenen Praxisferne eine gewisse Theorie- und Datenmüdigkeit, so dass die Cefahr von gegenseitiger Abschottung und wechselseitigem Verdruss heraufbeschworen wird.« (Möller 2003, S. 44; zit.n.: Becker 2011a, S. 19).

121 Becker zitiert hier Roth/Lynen von Berg/Benack (2003, S. 19).

122 Die Programme wurden im Rahmen dieser Studie somit (größtenteils) nicht mit eigenen erhobenen Daten untersucht. Dies hat unterschiedliche Gründe: Es lagen zu allen Programmen z.T. sehr umfangreiche Evaluationen vor und die absolute Anonymisierung der Programme wäre bei einer eigenen empirischen Untersuchung aufgrund ihres Modellcharakters schwerlich möglich gewesen; das Programm »DU HAST DIE MACHT « befand sich zudem zum Untersuchungsbeginn 2014 bereits in der Abwicklungsphase. Teilnehmende Beobachtungen waren 


\subsubsection{DU HAST DIE MACHT}

In dem Programm »DU HAST DIE MACHT«, das die RBS von 2010 bis 2015 förderte, verfolgte sie das Anliegen, »[s]oziale Medien konsequent für die politische Bildung zu nutzen«, womit sie gemeinsam mit ihren Partnern »im Jahr 2010 Neuland« betrat (Robert Bosch Stiftung 2017p). Mit DHDM sollen »junge Menschen über innovative Online-Aktionen und Bewegtbildproduktionen für das Thema Politik begeistert « (ebd.) werden. Für das Programm beauftragte die RBS die UFA Film und TV Produktion GmbH, die im Programm auch pädagogische Aufgaben übernahm. ${ }^{123}$ Als Zielgruppe wurden »junge Menschen zwischen 14 und 23 Jahren« ins Auge gefasst (dies.o.J.). Vor allem zu Beginn war es das Ziel, »politikferne Gruppen« (Besand/Birkenhauer/Lange 2013, S. 10) zu erreichen; mit der Zeit wurde jedoch eine Zielgruppenerweiterung vorgenommen (vgl. ebd., S. 154).

Mit dem Fokus auf Bewegtbild- und Online-Formaten wurden bei DHDM zahlreiche neue, mediale politische Bildungsformate erprobt. Dazu gehörten u.a. Video-Formate wie »Was sagt die Straße?«, bei dem zu verschiedenen aktuellen Themen Straßenumfragen durchgeführt wurden. ${ }^{124}$ Bei dem Wettbewerbsformat »RAPutation « konnten »Jugendliche in Rap-Form politisch Stellung beziehen « (Robert Bosch Stiftung 2017q). Des Weiteren bot DHDM auch eine »interaktive Video-Plattform für den Unterricht« an: »Teach and Show«, »die informative Videos von YouTube und anderen Videoportalen kategorisiert und kostenfrei für den (Schul-)Unterricht zur Verfügung stellt« (dies. 2017r). Gemeinsam mit der bpb wurden im Rahmen von DHDM »TenseInforms « und »BrainFed « als »Newsmagazine für jugendrelevante Nachrichten und Dauerbrennerthemen « mit den You-Tube-Stars Tense und DarkViktory entwickelt (vgl. dies. 2017s). »BrainFed« erhielt den Publikumspreis des Grimme Online Award 2016 (vgl. Grimme Online Award 2016). Auch wurde ein Jugendbeirat eingerichtet, der ein Jahr lang (2012 bis 2013) regelmäßig tagte und die Formatentwicklung beeinflusste; später wurde dieser durch den »YouTuber-Beirat ergänzt oder ersetzt « (Besand/Birkenhauer/Lange 2013, S. 160). Großer Wert wird im Programm auf die >Überparteilichkeit` gelegt:

(auf Anfrage) im Programm »Dialog macht Schule« nicht möglich - selbst die Evaluator*innen mussten auf diese weitestgehend verzichten: u.a. aus dem Grund, dass die Dialogmoderator*innen zurückgemeldet hätten, dass Hospitationen bei der ersten Evaluation (die durch ein anderes Forschungsteam durchgeführt wurde) als ablenkend und irritierend bei den Cruppen und Moderator*innen empfunden worden waren (vgl. Besand/Fuhrmann o.J., S. 45).

123 Diese Information ist einem Cespräch mit der damaligen Projektleiterin von DHDM bei UFA LAB, Anna Mauersberger, am 30. Juli 2014 in Berlin entnommen. Besand/Birkenhauer/Lange analysieren auch, »welche Herausforderungen sich im Kontext zentraler Ziel- und Aufgabenstellungen ergeben, wenn Angebote zur politischen Bildung von journalistisch qualifizierten Redaktionen entwickelt werden sollen.«(Besand/Birkenhauer/Lange 2013, S. 170)

124 Themen waren z.B. »Was ist typisch deutsch?«, »Homophobie«, »Ramadan: Der Fastenmonat der Muslime«, »Europa, Einwanderer und Sozialhilfe«, »Cannabis«, »Klimawandel«; die Clips finden sich auf YouTube: vgl. YouTube 2017. 
$\mathrm{Zu}$ Podien oder Programmveranstaltungen werden Vertreter*innen aller Parteien eingeladen. ${ }^{125}$

In der Evaluationsstudie zum Programm wird dieses insbesondere aus zwei Gründen als innovativ eingeordnet, nämlich aufgrund seiner Zielgruppenorientierung und der Formatwahl. So gäbe es immer noch kaum Angebote für »sowohl Jugendliche mit Migrationshintergrund [...] als auch Jugendliche mit Bildungsnachteilen (Besand/Birkenhauer/Lange 2013, S. 41). ${ }^{126}$ Hinsichtlich der Wahl der Vermittlungsform« stellen Besand/Birkenhauer/Lange den folgenden Bedarf fest:

»Obwohl die Nutzung von netzbasierten Medien zur niedrigschwelligen Vermittlung politischer Bildung an politik- bzw. bildungsferne Jugendliche eigentlich naheliegt, finden sich bislang kaum Beispiele für politische Onlineplattformen mit dieser Ausrichtung. Die meisten existierenden Web2.0-Angebote zur politischen Bildung richten sich derzeit noch immer an Multiplikatoren und Multiplikatorinnen [...].« (Ebd., S. 41f.)

Insgesamt werden starke Anerkennungspraxen bei DHDM deutlich: Unter Einbindung von YouTube und Facebook werden sprachlich und ästhetisch ansprechende Formate produziert (vgl. ebd., S. 84.); auch beliebte Musikstile wie Rap und jugendliche Vorbilder (Stars) werden einbezogen, indem sie die Schirmherrschaft für das Programm übernehmen. ${ }^{127}$ Oder es wird der Versuch unternommen, Jugendliche in die Formatentwicklung einzubinden (wie sich an dem Jugendbeirat zeigt).

Mittlerweile hat sich »[a] us DU HAST DIE MACHT [...] MESH COLLECTIVE entwickelt«, das ein »Projekt der UFA GmbH« ist (Meshcollective 2017a) und »Bewegbildformate auf YouTube mit Bildungsanspruch [produziert] [...]« (Robert Bosch Stiftung 2017p). Zu den »Partner[n] « gehören, neben der RBS und der bpb u.a. auch die Bertelsmann Stiftung, ${ }^{128}$ die Stiftung Mercator, ${ }^{129}$ die Joachim Herz

125 So waren z.B. zum Finale der zweiten RAPutation-Staffel im Jahr 2014 Politiker*innen der verschiedenen Bundestagsparteien eingeladen: »Katja Kipping (Die Linke) nimmt an der Garderobe die Jacken entgegen, Özcan Mutlu (Die Grünen) arbeitet am Merchandising-Stand und Gregor Gysi (Die Linke), Cansel Kiziltepe (SPD) und Jens Spahn (CDU) stehen hinter der Bar.« (Robert Bosch Stiftung 2017t)

126 Besand/Birkenhauer/Lange befinden: »Cemessen an der Zahl der Jugendlichen, die dieser durchaus heterogenen Zielgruppe zugerechnet werden müssen, sind die Angebote aber noch immer überschaubar.« (Besand/Birkenhauer/Lange 2013, S. 41)

127 Im Programmflyer werden als »Celebrity Friends « ein Rapper, eine Moderatorin, eine Sängerin, eine Soap-Darstellerin und ein Blogger genannt (vgl. Robert Bosch Stiftung o.J.).

128 Die Bertelsmann Stiftung hat z.B. ein Video zum Thema »Mitentscheiden und mithandeln in der Kita« im Rahmen des Programms »jung bewegt« beauftragt (vgl. Facebook 2017).

129 Mesh Collective hat für die Stiftung Mercator Videos für die Online-Kampagne »\#CheckDichAus « produziert, die wiederum »Teil des Programms Mercator Exchange« ist, »das die internationalen Austausch- und Begegnungsprogramme der Stiftung Mercator für Schüler, Jugendliche und Multiplikatoren bündelt« (Meshcollective 2017c). 
Stiftung oder die Adenauer Stiftung (vgl. Meshcollective 2017b). Damit ist auch ein Hinweis gegeben, wer sich die kostenintensiven innovativen Bildungsformate im Web 2.0 leistet bzw. auch leisten kann oder möchte.

Das Programm DHDM wurde in seiner Entstehungsphase zwei Jahre begleitend evaluiert. Die Evaluation wurde mit einer formativen Beratung des Projektteams verbunden, das heißt, dass Ergebnisse aus dem laufenden Forschungsprozess in die Projektarbeit gegeben wurden. ${ }^{130}$ Dadurch konnten Fehler frühzeitig erkannt und »Bedürfnisse der Zielgruppe« berücksichtigt werden (Besand/Birkenhauer/Lange 2013, S. 32). ${ }^{131}$ Die daraus entstandene Fallstudie möchte auf die »Chancen und Herausforderungen« politischer Bildung im Netz verweisen und aufzeigen, wie sich "politische Bildungsprozesse im Web 2.0 grundsätzlich besser auf Zielgruppen abstimmen lassen « (Besand 2013). ${ }^{132}$

Eine der »wichtigste[n]« Forschungsfragen der Evaluation ist die, »inwiefern es dem Projekt DU HAST DIE MACHT gelingt, die anvisierte Zielgruppe auch tatsächlich zu erreichen« (Besand/Birkenhauer/Lange 2013, S. 32). Damit seien des Weiteren die Fragen verbunden, ob die

»Angebote der Internetplattform DU HAST DIE MACHT tatsächlich geeignet [sind], spolitikferne ]ugendliche auf politische Fragen aufmerksam zu machen, mit ihnen authentisch und auf Augenhöhe über politische Fragen zu diskutieren, sowie sie langfristig an politische Partizipation heranzuführen?« (Besand 2013)

Um diese Fragen (z.B. nach der Zielgruppenerreichung) zu beantworten, wurde eine qualitative Untersuchung durchgeführt und »Jugendliche aus unterschiedlichen sozialen Gruppen im Hinblick auf ihre Wahrnehmung und Einschätzung des Projektangebots befragt « (ebd.).

In der Evaluation werden »drei relevante Perspektiven« eingenommen: die erste hinsichtlich des Angebots, die zweite hinsichtlich seiner Rezeption und die dritte hinsichtlich der Produktion (ebd.). Die erste Perspektive umfasst die Beurteilung aus fachdidaktischer Sicht und ist für die vorliegende Untersuchung besonders wichtig. Denn wie auch in der Evaluation festgestellt wird, ist »Innovation [...] als Gütekriterium von eher ambivalenter Bedeutung. Auch die Nähe zur Zielgruppe

130 Die Forschergruppe hat sich »regelmäßig (ca. alle 8 Wochen) mit der Redaktionsgruppe im UFA Lab zu sogenannten Resonanzgesprächen « getroffen (Besand 2013).

131 Das Forschungsteam hebt die Vorteile einer begleitenden Evaluation mit formativer Beratung (auf der Website des Lehrstuhls) hervor: »[...] wir [sind] der Überzeugung, dass sich eine Verbesserung der Instrumente politischer Bildung und damit nachhaltiger Innovation in diesem Feld nur entwickeln kann, wenn Ergebnisse möglichst frühzeitig in den Produktionsprozess zurückgespiegelt werden.«(Besand 2013)

132 Diese Ergebnisse können hier nicht ausführlich dargestellt werden, vgl. dazu: Besand/Birkenhauer/Lange 2013, S. $78 \mathrm{ff}$. 
und die Offenheit für Anregungen von außen machen an sich noch kein gelungenes Projekt aus.«(Besand/Birkenhauer/Lange 2013, S. 44)

Als »drei Klassen von Güteinstrumenten in der politischen Bildung « werden der »Beutelsbacher Konsens ${ }^{133}$ " ${ }^{3}$ Didaktische Prinzipien ${ }^{134}$ und »Bildungsstandards $\aleph^{135}$ herangezogen. Prinzipien kritisch-emanzipatorischer politischer Bildung und Didaktik werden von Besand/Birkenhauer/Lange somit nicht als Bewertungsmaßstäbe oder -grundlage herangezogen, was insofern nicht überrascht, als dass sie in der der fachdidaktischen Forschung eine eher randständige Rolle spielen.

Die Bewertung aus fachdidaktischer Sicht kann hier nur in gebotener Kürze wiedergegeben werden: Analog zu den Beurteilungskriterien schneidet DHDM z.B. bei »Adressatenorientierung/Schülerorientierung« sehr gut ab (vgl. ausführlich: Besand/Birkenhauer/Lange 2013, S. 47). So würden nicht nur aktuelle Themen jugendgerecht besprochen, sondern es fänden sich auch viele »jugendkulturelle Aspekte« (mit Stars, Bands, TV-Serien etc.) und unterhaltungsorientierte Beiträge. Hinsichtlich des Kriteriums der Einhaltung des Kontroversitätsgebots werde sich zwar bei der Informationsvermittlung um eben jene Kontroversität erfolgreich bemüht, z.B. über Pro- und Contra-Debatten, jedoch würden sich »Schwierigkeiten« beim »erste[n] und vornehmste[n] Ziel des Projekts « ergeben: »ugendliche zur politischen Partizipation anzuregen, d.h. sie zu aktivieren« (ebd., S. 50).

Wie Besand/Birkenhauer/Lange hervorheben, ist dies keineswegs eine triviale, sondern eine höchstrelevante Frage, da »[a]ktivierend [...] (insbesondere in der Umgebung des Web 2.0) eher pointierte Stellungnahmen als vorsichtig formulierte, um Ausgewogenheit ringende Hintergrundbeiträge [wirken].« (Ebd.) Bei »Themenstruktur und Ausrichtung der weniger auf Information als auf Beteiligung und Aktivierung ausgerichteten Angebote« von DHDM ergäbe sich »ein eher kritisches Bild, was Kontroversität und Überwältigung« betrifft (ebd.). So könne bei bestimmten Themen

»eine klare, auch weltanschauliche Positionierung des Projekts festgestellt werden - nämlich für Umweltschutz und nachhaltiges Verhalten, gegen Kernenergie, für Integration und Vielfalt in der Cesellschaft sowie für eine Steigerung der Ausgaben im Bildungsbereich.« (Ebd.)

133 Zum Beutelsbacher Konsens (BK) und der aktuellen Debatte über diesen: vgl. Kapitel 2.1.2.

134 Als didaktische Prinzipien werden Adressatenorientierung, Problemorientierung, Exemplarisches Lernen, Handlungsorientierung, Kontroversität, Wissenschaftsorientierung genannt (vgl. Besand/Birkenhauer/Lange 2013, S. 45).

135 Mit Bildungsstandards wird sich auf die 2004 von der Cesellschaft für Politikdidaktik und politische Jugend- und Erwachsenenbildung (CPJE) veröffentlichten Standards für die politische Bildung in der Schule bezogen: politische Urteilsfähigkeit, politische Handlungsfähigkeit und methodische Fähigkeiten (vgl. Besand/Birkenhauer/Lange 2013, S. 45). 
Um die Rezeption bei Jugendlichen zu erheben (Perspektive 2) wurden »Jugendliche mit unterschiedlichen Bildungsbiographien aus unterschiedlichen sozialen Kontexten « befragt (ebd., S. 59). ${ }^{136}$ Insgesamt kann hinsichtlich der Ergebnisse zur Rezeption hervorgehoben werden, dass »[n]ahezu alle« der befragten Jugendlichen »[...] es toll finden, dass es >so etwas wie DU HAST DIE MACHT überhaupt gibt'« (ebd., S. 61). Die Jugendlichen - egal aus welcher Gruppe - würden

»den Ansatz [honorieren], etwas speziell für Jugendliche zu machen und sie (also die Jugendlichen) selbst dabei immer wieder zu Wort kommen zu lassen. In vielen Interviews kam zum Ausdruck, dass vor allem die Bereitschaft auf junge Leute zuzugehen und ihnen zuzuhören eine der Crundstärken des Projekts [...] ist. $«(\text { Ebd.) })^{137}$

Besonders gut kam an, dass DHDM mit Bewegtbild arbeitet:

$»[$...] dieses Medium [wurde] von allen Jugendlichen stets als überaus positiv hervorgehoben. Videos finden alle Jugendlichen völlig unabhängig von sozialem Hintergrund oder Bildungshintergrund wunderbar. Sie schauen sie konzentriert an und finden über sie Zugänge zu Themen, die sie sich über Texte niemals angeeignet hätten. Insbesondere Jugendliche der Cruppe 3 sind [...] fast ausschließlich über diesen Kanal erreichbar.« (Ebd., S. 74; Herv. i. O.) ${ }^{138}$

In der dritten Perspektive wurden die »Produktionsperspektiven und -bedingungen « von DHDM näher betrachtet. Auch dies passiert in der politischen Bildung eher selten und ist für die Weiterentwicklung eben jener hochinteressant, insbesondere da die Frage danach gestellt wurde, wie die Zielgruppe selbst am Produktionsprozess beteiligt werden kann (vgl. ebd., S. 145). Um zielgruppenadäquate Formate zu entwickeln, wurden im Programm verschiedenen »Strategien zur Überwindung der Distanz zur Zielgruppe« nachgegangen (ebd., S. 159-163). Eine davon ist der »[i]nstitutionalisierte Kontakt mit der Zielgruppe«, z.B. durch den Jugendbeirat

136 Dafür haben sie einen »zweidimensionalen Merkmalsraum« benutzt, der »Bildungshintergrund« und »Nähe oder Ferne zur Politik« abbildet; der Bildungshintergrund wird dabei vorrangig an der Schulform festgemacht (vgl. Besand/Birkenhauer/Lange 2013, S. 59f.). Besand/Birkenhauer/Lange beschreiben, dass die Klassifizierung als spolitikferne*r Jugendliche"r< schwierig war, da es z.B. >benachteiligte Jugendliche sibt, die offensichtlich großes Interesse an politischen Themen haben - als Beispiel nennen sie u.a. ein Mädchen, das aus prekären Lebensverhältnissen stammt und »Kontakt zur rechten Szene pflegt«, sich für »Ceschichte, den zweiten Weltkrieg [...], für Migration und das NPD-Verbot« interessiert (ebd., S. 59).

137 Es gab auch Kritik vonseiten der Jugendlichen am Programm DHDM, z.B. hinsichtlich der »Fülle der Informationen « und der »Übersichtlichkeit des Angebots « (Besand/Birkenhauer/Lange 2013, S. 61). Die Evaluator*innen haben auch den Eindruck, dass Jugendliche aus allen Gruppen sich eher oberflächlich mit der Seite beschäftigt haben (vgl. ebd., S. 63).

138 Zu Cruppe 3 gehören Jugendliche, die die Hauptschule besuchen oder/und zu»Risikogruppen « gehören (vgl. Besand/Birkenhauer/Lange 2013, S. 60). 
bzw. YouTuber-Beirat (ebd., S. 160). Die Mitglieder der Beiräte sind jedoch »eher als engagierte und bildungserfolgreiche Jugendliche $\mathrm{zu}$ beschreiben «, weshalb die Evaluator*innen hervorheben, dass »Sorge dafür getragen werden « müsse, »dass die Beiräte sich nicht $\mathrm{zu}$ sehr von der Zielgruppe unterscheiden« - auch wenn durch sie immerhin »eine Brücke in die entsprechenden Altersgruppen« geschlagen werden könne (ebd.).

\subsubsection{Dialog macht Schule}

Im Jahr 2009 initiierten die RBS und die bpb das Modellprojekt »Jugend, Religion, Demokratie: Politische Bildung mit Jugendlichen in der Einwanderungsgesellschaft «, ${ }_{139}$ das heute unter dem Namen »Dialog macht Schule« (DmS) firmiert. Das Programm richtet sich an die "neue Generation von Kindern aus Einwandererfamilien«, von denen »[v]iele [...] in sogenannten sozial benachteiligten, oft auch bildungsfernen Familien auf[wachsen]« (Robert Bosch Stiftung 20170). Da die »klassischen Methoden politischer Bildung [...] an diesen Kindern und Jugendlichen häufig vorbei[gehen]«, soll »Dialog macht Schule« diese »direkt und auf neue Weise« ansprechen:

»Im Mittelpunkt stehen die Dialoggruppen, die aus 10 bis maximal 15 Schülern ab Klasse sieben bestehen, vornehmlich aus Einwandererfamilien. Die Gruppen treffen sich wöchentlich über einen Zeitraum von zwei Jahren, entweder im Rahmen freiwilliger AGs oder als Teil des Politik- oder Ethikunterrichts. Celeitet werden sie von sogenannten Dialogmoderatoren, jungen Erwachsenen mit Migrationshintergrund, die für ihre Aufgabe in Seminaren und Workshops ausgebildet und vernetzt werden.«(Ebd.)

Momentan besteht DmS an fünf Standorten (Berlin, Hamburg, Hannover, Stuttgart, Wuppertal) und erreicht rund 750 Schüler*innen (Stand: März 2015) (vgl. Dialog macht Schule 2017a), bisher wurden 90 Dialogmoderator*innen ausgebildet (vgl. Robert Bosch Stiftung 20170). Zum Teil findet DmS im Rahmen des Regelunterrichts statt (vgl. Besand/Fuhrmann o.J., S. 123) und ist mit der Vergabe von Noten durch die Dialogmoderator*innen verbunden (vgl. ebd., S. 58, 66).

2013 gründeten zwei ehemalige Dialogmoderatoren aus dem Modellprojekt heraus die »Dialog macht Schule gGmbH« (Robert Bosch Stiftung 20170). Der »Verbreitungsansatz« von DmS basiert nun auf einem »Social Franchise System« (Dialog macht Schule 2017a) und folgt der »Vision, bis 2020 jährlich 1.000 Dialogmoderatoren auszubilden, um so bis zu 25.000 bildungsbenachteiligte Schüler zu erreichen und deren Startchancen zu verbessern« (Teucher 2014). Das Programm

139 Das Modellprojekt startete in Schulen in Berlin-Neukölln und Stuttgart und wurde entsprechend in Kooperation mit der Stadt Stuttgart und dem Bezirksamt Berlin-Neukölln durchgeführt (vgl. Robert Bosch Stiftung 20170). 
wird durch unterschiedliche Partner gefördert. ${ }^{140}$ Die Bundeschirmherrschaft hat Aydan Özoguz, die Beauftragte der Bundesregierung für Migration, Flüchtlinge und Integration, übernommen (vgl. Dialog macht Schule 2017b).

Laut Evaluationsbericht sei ein Alleinstellungsmerkmal von DmS, dass es ein Mentoringprogramm ist - solche gäbe es zwar zahlreich im Bildungsbereich, aber bisher keines »mit klarer Ausrichtung und Schwerpunkt auf politische Bildung« (Besand/Fuhrmann o.J., S. 20). Zu den Voraussetzungen dafür, dass man Dialogmoderator*in werden kann, gehört, dass man Student*in ist und eine "Einwanderungsbiographie und/oder interkulturelle Erfahrungen« hat (Dialog macht Schule 2017a). Man geht davon aus, dass »Menschen mit Migrationshintergrund [...] leichter Zugang zu anderen Menschen mit Migrationshintergrund [schaffen]«(Besand/Fuhrmann o.J., S. 16).

DmS betrachtet sich als innovativ, da es mit einem »dialogisch-partizipativen Ansatz an der Schnittstelle von schulischer und außerschulischer politischer Bildung « arbeitet (Dialog macht Schule 2017a). ${ }^{141}$ Dieser Ansatz beruht vor allem darauf, dass Jugendliche eigene Themen einbringen können. Man lege einen »weiten Politikbegriff zu Grunde« (ebd.), die »Themen und Interessen der Jugendlichen« sollen »im Vordergrund stehen« (dies. 2017c) und man will »den Fokus gezielt auf einen lebensweltlichen Zugang« legen (dies. 2017d). Auf der Website heißt es dazu:

»Dialogmoderator*innen leisten wahre Detektivarbeit, wenn es um das Aufspüren der versteckten politischen Interessen ihrer Schüler*innen geht. So wird zum Beispiel aus dem Engagement im Sportverein eine Diskussion über gesellschaftliche Teilhabe und aus dem Streit mit der älteren Schwester ein Austausch über die Unumgänglichkeit von Konflikten, die Notwendigkeit von Kompromissen und dem Verständnis für andere Perspektiven in einer Demokratie. Themen wie Identität, Heimat, kulturelle Vielfalt, Stereotype, Geschlechterrollen und viele mehr, bieten (nicht nur für unsere Schüler*innen.) [sic!] Raum, die eigenen Perspektiven zu erweitern, Rollenzuschreibungen zu überwinden und ein erweitertes Reflexionsvermögen zu entwickeln.«(Ebd.)

In der Evaluation wird betont: "Solche aufsuchenden dialogisch orientierten Formate sind im Bereich der politischen Bildung bislang noch eher Mangelware [...] (Besand/Fuhrmann o.J., S. 6). So müsse allein der Fakt, dass man bei DmS ohne

140 Auch das Software-Unternehmen SAP förderte »[...] mit 15000 Euro [...] die Erweiterung des Berliner Schulnetzes von >Dialog macht Schule'« (Oeing 2014). Dass SAP ein Programm fördert, das sich für Demokratie einsetzt, ist insofern bemerkenswert, als dass SAP im eigenen Konzern lange Zeit versucht hat, demokratische Mitbestimmung, z.B. durch einen Betriebsrat, zu verhindern (vgl. FAZ 2006).

141 Auch die Evaluation bestätigt nach 12-monatiger wissenschaftlicher Begleitung, dass das Modellprojekt ein »innovatives und überaus gelungenes Format«sei (Besand/Fuhrmann o.]., S. 4). 
Agenda und mit freiem Dialog arbeiten will, im Kontext Schule »(leider) noch immer als innovativer Ansatz bewertet werden« (ebd., S. 16f.). Dass die Schüler*innen den Eindruck haben, dass mit DmS herausgefunden werden soll, »was Jugendliche denken«, sei damit »für ein dialogisch orientiertes Projekt kein schlechter Befund «, so die Autorinnen der Evaluationsstudie (ebd., S. 55). ${ }^{142}$ Als Alleinstellungsmerkmal von $\mathrm{DmS}$ wird somit auch der freie Dialog in Kombination mit politischen Inhalten gewertet (vgl. ebd., S. 116).

Besand/Fuhrmann resümieren in der Evaluation zum Programm, ${ }^{143}$ dass es zu den Stärken des Programms gehöre, dass Jugendliche mit Migrationshintergrund erreicht werden (vgl. ebd., S. 112). Auch in dieser Evaluation wird zudem deutlich, dass die jugendlichen Teilnehmer*innen das Projekt »nicht selten geradezu enthusiastisch [beurteilen] «, obwohl sie zu Projektbeginn skeptisch waren (ebd., 59). Dabei

»scheint es [...] von zentraler Bedeutung zu sein, dass in den Dialoggruppen offen auf Fragen und Probleme der Jugendlichen reagiert wird und damit eine selbstbestimmte Themensetzung möglich ist. Dieser Aspekt wird von den Jugendlichen als zentraler Pluspunkt des Projekts bewertet. (Ebd.)

Wichtig ist insbesondere auch die umgekehrte Erkenntnis, dass »[i]n Gruppen, in denen dies nicht oder für ihre Begriffe zu selten praktiziert wird, [...] sich die Schülerinnen und Schüler entsprechend auch deutlich kritischer [verhalten]« (ebd.). Die >Themenoffenheit bei DmS ist somit als ein besonders innovatives Element in Hinblick auf die Frage, wie Jugendliche erreicht werden können, zu bewerten. Deshalb sollen hier auch einige Ergebnisse der Evaluation zur Themenfindung innerhalb der Dialoggruppen wiedergegeben werden.

Besand/Fuhrmann weisen (unter Bezug auf Becker 2000) darauf hin, dass in der außerschulischen politischen Bildung oft »[u]npräzise Zieldefinitionen

142 Besand/Fuhrmann analysieren auch, wie die Schüler*innen »die Zielgruppe des Projekts [sehen] « (Besand/Fuhrmann o.J., S. 81). Ein*e interviewte*r Schüler*in antwortet in diesem Kontext auch: »Also ich glaube, die wollten uns halt/die wollten halt wissen, wie die Jugend heutzutage ist. Wie die meinen, wie sie denken/» (ebd.).

143 Die Evaluation fokussiert die Alleinstellungsmerkmale und Gelingensbedingungen des Programms sowie die Weiterbildung der Dialogmoderator*innen (vgl. Besand/Fuhrmann o.]., S. 37). Die Ergebnisse können hier nicht ausführlich dargestellt werden und sind z.T. in die Darstellung des Formats eingeflossen. Methodisch wird ein qualitativer Ansatz in der Evaluation verfolgt: einmal eine fachdidaktische Analyse, um die Qualität des Projekts zu beurteilen, und zum zweiten eine empirische Analyse, bei der es um die Wahrnehmung des Programms durch die Zielgruppe und um die beteiligten Projektakteure geht (vgl. ebd., S. 43; zum Einsatz der methodischen Verfahren: vgl. ebd., S. 43ff.). Der (unveröffentlichten) Evaluation von Besand/Fuhrmann ist eine andere Evaluation vorausgegangen, deren Ergebnisse in einer Publikation veröffentlicht wurden (vgl. Bundeszentrale für politische Bildung/Robert Bosch Stiftung 2011). 
oder unkonkrete Leitbilder « zu finden seien (Besand/Fuhrmann o.J., S. 63). Auch DmS habe eine solche "unscharfe Zielbeschreibung « und so sei den Dialogmoderator*innen z.T. unklar, »welchen Auftrag sie nun eigentlich haben und welche Feinziele sich hinter großen Begriffen wie bspw. >Bewusstsein für Demokratie und politische Partizipation entwickeln< [...] verbergen« (ebd.). Das habe auch gute Effekte, da sich die Dialogmoderator*innen dadurch »sehr stark auf die [...] Jugendlichen einlassen« (ebd., S. 63f.).

In der Evaluation wird u.a. die Frage beleuchtet, wie ein Sachverhalt zum Thema in einer Dialoggruppe wird. Hierbei kristallisierten sich verschiedene Strategien heraus: Das »[A]blauschen« von Themen, z.B. durch eine >Wie-geht's-mirRunde $<$, das direkte Erfragen von Themen, das Einbringen von Themen durch Dialogmoderator*innen, wenn sie davon ausgehen, dass diese vermeintlich interessant seien oder auch das Aufgreifen von Themen, die schon im Unterricht behandelt wurden (vgl. ebd., S. 94-97). Bei der Frage, wie man nun zur politischen Dimension kommt, würden die Dialogmoderator*innen »[...] zwischen den Zielpolen [jonglieren], einen freien Dialog mit Jugendlichen einzugehen und ihnen neue Einsichten in politisch-gesellschaftliche Zusammenhänge zu eröffnen« (ebd., S. 98). Man benutze einen weiten Politikbegriff un d nehme Bezug zu "grundlegenden politischen Kernfragestellungen oder Kernkonzepten« (ebd.). ${ }^{144}$

Insgesamt stellen Besand/Fuhrmann deutliche Weiterbildungsbedarfe fest, wie man zur politischen Dimension kommt (vgl. ebd., S. 100). Dass politische Bildungsforschung nur selten der Formulierung konkreter Weiterbildungsbedarfe dient, wurde eingangs in diesem Kapitel erwähnt. Die Evaluation zu DmS formuliert einen solchen Weiterbildungsbedarf für die Dialogmoderator*innen zur »Reflexion über politische Bildung« (ebd., S. 148). ${ }^{145}$ Ein Entwurf für ein Weiterbildungsseminar beinhaltet deshalb auch eine Einheit, in der »[e]in klarer Bezug zur politischen Bildung [...] hergestellt werden [soll] « (ebd., S. 152). ${ }^{146}$

144 Als Bezug geben Besand/Fuhrmann. die »Autorengruppe Fachdidaktik«an (vgl. Autorengruppe Fachdidaktik 2011). Einige Dialogmoderator*innen würden an Basiskonzepte der politischen Bildung anschließen (vgl. Besand/Fuhrmann o.J., S. 99).

145 Dabei würden jedoch die Fortbildungen zur politischen Bildung von den Dialogmoderator*innen »scheinbar als zu abstrakt und fern von der Arbeitsrealität der Dialoggruppen empfunden« (Besand/Fuhrmann o.J., S. 148). Die Fachdidaktikerinnen befinden jedoch, dass man es »genauer betrachtet [...] hier allerdings lediglich mit einem Imageproblem der politischen Bildung zu tun $[. ..] \ll$ habe (ebd.).

146 In bestehenden Konzepten für die Weiterbildung der Dialogmoderator*innen ließen sich bereits »didaktische Prinzipien und Basiskonzepte« finden (Besand/Fuhrmann o.J., S. 169). 


\subsubsection{Lernort Stadion}

Seit 2010 fördert die Robert Bosch Stiftung zusammen mit der DFL Stiftung (ehemals Bundesliga Stiftung) ${ }^{147}$ das Programm »Lernort Stadion«, bei dem aktuell 12 Lernzentren in ganz Deutschland gefördert werden. ${ }^{148}$ Diese bieten mehrtägige Workshops, Projekttage oder längerfristige Seminare für Jugendliche an (vgl. Robert Bosch Stiftung 2013a, S. 2). Themen sind hierbei u.a. "Migration, Rassismus und (Rechts-)Extremismus, Zivilcourage, Umweltschutz, Mobbing, Homophobie, Umgang mit (sozialen) Medien, Gewaltprävention [...]« (ebd.). »Lernort Stadion« ist nach dem »Vorbild « der aus England stammenden »Study Support Centres« gestaltet, bei denen Bildungseinrichtungen und Fußballclubs zusammenarbeiten (ebd., S. 4). Aus dem Programm heraus hat sich im Oktober 2014 der »Lernort Stadion e.V.« gegründet (vgl. dies. 2017m), der als »Verbund der Lernzentren« fungiert (Lernort Stadion 2017a).

Die Lernzentren sind bei den Fanprojekten angesiedelt, die dafür als »Schnittstelle zwischen Fußball und politischer Jugendarbeit [...] in der idealen Rolle« seien (Robert Bosch Stiftung 2013a, S. 4). Jedes Lernzentrum hat ein »eigenes Profil«, das an den jeweiligen Standort angepasst ist (dies. 2017m). Oftmals wird mit den Fußball-Clubs, den CSR-Stiftungen der Clubs (z.B. BVB-Stiftung im BVBLernzentrum in Dortmund) und mit weiteren Partnern (z.B. Arbeit und Leben, AWO) kooperiert. Im Jahr 2012 wurde das Modellprojekt im bundesweiten Wettbewerb »365 Orte im Land der Ideen $\aleph^{149}$ u.a. für seinen »konzeptionelle[n] Projektansatz« ausgezeichnet (dies. 2013a, S. 4).

Das Programm zeichne sich durch eine »niedrigschwellige Herangehensweise, die lebensweltbezogene Themenauswahl und [...] wertschätzende[n] Umgang mit den Jugendlichen« aus (Bundesliga Stiftung 2016c). Das Stadion, Fußball als Sport bzw. auch ein bestimmtes Umfeld sollen motivieren, ein »Erlebnis« darstellen und eine "positive Haltung" im Bildungssetting hervorrufen (Robert Bosch Stiftung $2017 \mathrm{~m}$ ). Die Angebote würden sich »deutlich vom oft als frustrierend empfundenen Schulalltag [unterscheiden] « (dies. 2013a, S. 4). Im Stadion will man

»exemplarisch veranschaulichen [...], wie Politik in viele unterschiedliche Bereiche des alltäglichen Lebens hineinwirkt. Ansatz des Projekts ist es, diese Zusammenhänge vor Ort ganz praktisch aufzuspüren und sie für Jugendliche nachvollziehbar zu machen.«(Ebd.)

147 Die DFL Stiftung wird im Kapitel 4.3.3.4 ausführlicher betrachtet.

148 Die Standorte sind in: Dortmund, Bochum, Bremen, Berlin, Bielefeld, Celsenkirchen, Frankfurt, Dresden, Rostock, Braunschweig, Nürnberg, Stuttgart, Wolfsburg (vgl. Robert Bosch Stiftung 2017m).

149 »Die Initiative >Deutschland - Land der Ideen< ist die gemeinsame Standortinitiative der Bundesregierung und der deutschen Wirtschaft, vertreten durch den Bundesverband der Deutschen Industrie (BDI).«(Land der Ideen 2017) 
Als Schlüsselfaktor wird dabei der "privilegierte Zugang zum Stadion mit der Nähe zum jeweiligen Club, zur faszinierenden Fußballwelt und zum sheiligen Rasen'« gesehen (ebd.). Dies bedeute zudem »eine enorme materialisierte Wertschätzung« (ebd., S. 4f.). In den Workshops kämen nicht nur »unterschiedliche Methoden, Übungen und Spiele zum Einsatz«, sondern auch »sportpädagogische Angebote, künstlerische Ansätze mit Musik, Graffiti und Theater« (ebd., S. 5).

Das Programm erreicht jedes Jahr rund 6000 Jugendliche (Bundesliga Stiftung 2016c). Obwohl es "am Grundprinzip der Freiwilligkeit und den Grundpfeilern der informellen Bildung « ausgerichtet ist (Robert Bosch Stiftung 2013a, S. 5), kommen die Jugendlichen jedoch oftmals im Rahmen des Schulunterrichts. Die »Gruppe im Schulverband« hat »Anwesenheitsplicht«, aber entscheide selbst über »aktive Beteiligung am Programm« (ebd., S. 6).

Als Zielgruppe sollen »Schülerinnen und Schüler, v.a. von Hauptschulen der Jahrgangsstufen sieben bis zehn, sowie fußballbegeisterte Jugendliche und junge Erwachsene aus der Fanszene« erreicht werden (Robert Bosch Stiftung 2017m). In weiteren Programmbeschreibungen werden diese auch als »Jugendliche mit und ohne Migrationshintergrund (Lernort Stadion 2017b) und als "sozial benachteiligte und bildungsferne Jugendliche« (Robert Bosch Stiftung 2013a, S. 4) beschrieben. Wie auch die beiden anderen Modellprojekte will »Lernort Stadion« somit »die Jugendlichen ansprechen, die von traditionellen Bildungsangeboten nur selten erreicht werden « (Bundesliga Stiftung 2012, S. 19). ${ }^{150}$ Dabei soll »die Nähe zu den Clubs« helfen: »Wir wissen doch, dass die Schüler nicht zum Fanprojekt wollen, sondern zu Werder!'«, betont ein Pädagoge vom »Lernort Stadion« (ebd.).

Die RBS hat (in Kooperation mit der DFL Stiftung) eine Methodensammlung mit einem Begleitheft für die Pädagog*innen an den Lernzentren herausgegeben (vgl. Robert Bosch Stiftung 2013a, b). Die Methodensammlung mit hochwertigem Erscheinungsbild hält zahlreiche Übungsanleitungen für mögliche Workshops bereit; einige Methoden wurden auch von anderen Bildungsträgern übernommen, darunter gewerkschaftsnahe wie das DGB-Bildungswerk Thüringen (vgl. dies. 2013b, S. 70) oder Arbeit und Leben Bielefeld (vgl. ebd., S. 152). Im Begleitheft finden sich nicht nur Hintergrundinformationen zum Projekt und zum politischen Bildungsverständnis, sondern auch Vorlagen für die Arbeit (wie z.B. Teilnehmendenbescheinigungen), Workshop-Beispiele und Informationen über jedes Lernzentrum. ${ }^{151}$

150 Oder wie es ein Pädagoge vom Bremer Fanprojekt »schmunzel[nd]« ausdrückt: Die Jugendlichen, »)die sich nie in der Volkshochschule oder bei der Landeszentrale für politische Bildung in einen Kurs einschreiben würden'«(Bundesliga Stiftung 2012, S. 19).

151 In dem Begleitheft wird auch der hohe Stellenwert der Öffentlichkeitsarbeit im Programm »Lernort Stadion« deutlich: So ist z.B. eine Einverständniserklärung für die Eltern zu finden, die darüber informiert, dass während der Teilnahme »zu Dokumentationszwecken, Außendarstellung des Angebots und Veröffentlichung ggf. Foto-, Film- und Tonaufnahmen [...]« angefer- 
Als Schirmherr für das Programm »Lernort Stadion« konnte Oliver Welke gewonnen werden, der nicht nur als Sportmoderator bekannt ist, sondern auch das ZDF-Satire-Magazin »heute-show « moderiert, das als gesellschaftskritisch gilt, da z.B. Lobbyismus oder Ungleichverteilung von Reichtum angeprangert werden. ${ }^{152}$ Mit ihm als Schirmherrn können die beiden unternehmensnahen Stiftungen, RBS und DFL Stiftung, hohe Glaubwürdigkeit für ihr Engagement in der politischen Bildung mit >benachteiligten Jugendlichen gewinnen.

Das Programm wurde wissenschaftlich begleitet und evaluiert vom Centrum für angewandte Politikforschung (CAP). Ein Zwischenbericht von 2013, in den Einsicht genommen werden konnte, fasst erste Untersuchungsergebnisse zusammen: Es werden

»die Ergebnisse der positiven Zustimmungsdaten der beteiligten Jugendlichen anhand der ermittelten Wirkungsfaktoren jeweils Parameter spezifisch (gemäß Alter, Ceschlecht, Bildungs- und Migrationshintergrund sowie mit Blick auf die Fußballaffinität der Jugendlichen) dargestellt.« (Feldmann-Wojtachnia 2013, S. 10)

Damit sollen u.a. für »die Weiterentwicklung des Projekts interessante Hinweise« geliefert werden (ebd.). In einer zweiten Evaluationsphase sollten dann »qualitative Interviews mit allen Projektbeteiligten« geführt werden (ebd.).

Die Evaluation hält fest, dass sich »[a]lle Programme [...] an bildungsferne und sozialschwache Jugendliche, größtenteils aus dem Haupt-, Förder- und Berufsschulbereich [richten], die als Schulklassen an den Programmen teilnehmen « (ebd., S. 3). Auf der Basis von 1.500 Befragungsteilnehmenden zeigt die Evaluation (vgl. ebd., S. 6), dass rund 30 Prozent der Jugendlichen »einen - zumeist türkischen Migrationshintergrund an[geben], der tatsächliche Anteil liegt jedoch schätzungsweise bei $60 \%$, bei einigen Schulklassen sogar bei $80 \%$ bis $100 \%$ (ebd., S. 3). Weiterhin würden

»60\% der Jugendlichen [...] aus der Haupt- bzw. Mittelschule, $10 \%$ aus der Förderschule, $20 \%$ aus der Berufsschule und derzeit ca. $10 \%$ aus anderen Bereichen mit teilweise anderen Bildungshintergründen (freie Gruppen, teilweise aus dem Sport) $[$ kommen].« (Ebd.)

Die Rezeption des Programms bei den Jugendlichen wird anhand verschiedener Kategorien gemessen (vgl. Abb. 3). Nur eine von elf genannten im Bericht betrifft

tigt werden (Robert Bosch Stiftung 2013a, 22). Die Eltern sollen sich u.a. damit einverstanden erklären, dass Aufnahmen von ihrem Kind gemacht sowie bearbeitet, zeitlich unbegrenzt gespeichert und veröffentlicht werden können (vgl. ebd.).

152 Die »heute-show « gilt als Sendung, die »der Kritik an Politik und Gesellschaft im Medium Fernsehen einen eigenen Platz« verschafft (Digitalfernsehen 2012). 
die politische Bildung und überprüft, ob die Jugendlichen »durch den [sic!] Programm gelernt [haben], Bürgersinn zu entwickeln« (Feldmann-Wojtachnia 2013, S. 9). Was genau damit gemeint ist, wird in dem Zwischenbericht nicht näher erläutert. Analog zu der Erhebung hat die Programmteilnahme aber 85 Prozent »Spaß gemacht« und 69 Prozent »fanden die Inhalte interessant« (ebd.). Die RBS resümiert unter Bezug auf die Evaluation:

»Die große Nachfrage nach den Angeboten von Lernort Stadion und die guten Ergebnisse der wissenschaftlichen Begleitung legen nahe, dass dieses Projekt eine Leerstelle innerhalb der politischen Bildung füllt.« (Robert Bosch Stiftung 2013a, S. 7)

Abbildung 3: Evaluationsergebnisse Lernort Stadion: Generelle Bewertung und Wirkung des Programms Lernort Stadion auf die Jugendlichen

85\% der Teilnehmenden hat das Programm im Ganzen Spaß gemacht,

$82 \%$ würden das Angebot anderen Jugendlichen weiterempfehlen,

> $79 \%$ der Jugendlichen haben das Stadion von einer neuen Seite kennengelernt,

$>75 \%$ haben durch den [sic] Programm gelernt, Bürgersinn zu entwickeln,

$>69 \%$ der Jugendlichen fanden die angebotenen Inhalte interessant,

$>69 \%$ glauben, das Gelernte in den Alltag transferieren zu können,

$65 \%$ schätzen es, bei den Angeboten selbst aktiv geworden zu sein,

64\% wurden im Lernzentrum zum Nachdenken und zur Diskussion motiviert,

$64 \%$ haben die Angebote zu Sport und Bewegung gefallen,

$56 \%$ der Jugendlichen wünschen sich, im Kontakt mit dem Angebot zu bleiben,

$55 \%$ der Teilnehmenden hätten sich eine längere Dauer gewünscht.

Quelle: Feldmann-Wojtachnia 2013, S. 9 (Herv. i. O.)

\subsubsection{Bildungsziele}

Aus den bisherigen Ausführungen ist deutlich geworden, dass die RBS (z.T. gemeinsam mit der bpb) tatsächlich viele Innovationsbedarfe aufgreift: hinsichtlich der Frage, wie >benachteiligte Jugendliche durch politische Bildungsangebote erreicht werden können. ${ }^{153}$ Auf was zielen jedoch die Programme? Setzen sie sich mit

153 Auch wenn z.T. eine Zielgruppen-Entgrenzung vorgenommen wurde: So sei seit Anfang 2013 das Programm DmS an allen Schulen der Sekundarstufe möglich (vgl. Besand/Fuhrmann o.]., S. 82). In Berlin liegt zwar das »Hauptaugenmerk auf ১sozialräumlich klar benachteiligte Stadtteile'«, damit gibt es einen »Vorbehalt der sozialen Lage«, aber »Bildungsferne'« ist kein »Alleinstellungsmerkmal« (ebd.; Besand/Fuhrmann zitieren hier aus einem internen Projektdo- 
sozialen Ungleichheitsverhältnissen auseinander? Diese Fragen soll nachfolgend explorativ vor allem an den Konzeptionen der Programme »Dialog macht Schule« und »Lernort Stadion« nachgegangen werden, da das Programm DHDM bereits abgeschlossen ist.

Soziale Ungleichheit wird als gesellschaftliches Problem in den Programmbeschreibungen oder Evaluationen zunächst einmal angesprochen. Z.B. wird in einer Publikation über »Dialog macht Schule« aus dem Jahr 2011 der »Zusammenhang zwischen Bildungsarmut, sozialer Herkunft und einer Einwanderungsbiographie« (Bundeszentrale für politische Bildung/Robert Bosch Stiftung 2011, S. 7) benannt und festgehalten, dass »die (politische) Bildungsbeteiligung « von Kindern und Jugendlichen aus Einwandererfamilien »zu einer dringlichen Zukunftsaufgabe « werde (ebd.). In der Zielgruppenbeschreibung wird die soziale Lage benannt und betont, dass "sozial benachteiligte Schüler*innen aus meist Einwandererfamilien direkt in Schulen im sozialen Brennpunkt ${ }^{154}$ angesprochen werden sollen (Dialog macht Schule 2017d). Operationalisiert wird die Zielgruppenansprache dadurch, dass man

»in der Praxis von der Annahme aus[geht], dass die für das Projekt relevanten Jugendlichen in Stadtteilen mit hoher Arbeitslosigkeit und/oder hohem Anteil an Bevölkerung mit Migrationshintergrund zu finden sind.«(Besand/Fuhrmann o.]., S. 82)

Die soziale Lage und die oft auch materiell schwierige Situation der Jugendlichen werden in der Publikation von 2011 dagegen nicht vertieft analysiert oder problematisiert, auch wenn sie z.B. in den Berichten aus der Praxis immer mal durchschimmern. ${ }^{155}$ Auch in den Programmbeschreibungen $\mathrm{zu}$ "Lernort Stadion « findet sich keine nähere Betrachtung der Lebenssituationen der Jugendlichen, obwohl auch hier die sozial prekäre Lage zumindest benannt wird. So heißt es im Begleitheft der Methodensammlung des Programms:

»Das Konzept ist auf Jugendliche ausgerichtet, die häufig in finanziell sowie sozial prekären Verhältnissen leben und dadurch gesellschaftlich benachteiligt oder bereits von vielen Entwicklungschancen ausgeschlossen sind. Teilhabe ist für sie nicht nur wegen eines einzelnen Hindernismerkmals, sondern aufgrund

kument). Die »Zielgruppe wird damit weiter interpretiert als ursprünglich vorgesehen [...]« (ebd.).

154 In der Evaluation ist auch die Rede von »sozio-ökonomisch benachteiligten Jugendlichen« (Besand/Fuhrmann o.]., S. 6).

155 Zum Beispiel, wenn in einem Interview ein Lehrer darauf hinweist, dass einige Schüler*innen »jobben [müssen], das heißt, ein Teil ihrer Kraft ist dadurch gebunden, dass sie für ihren eigenen Bedarf oder auch für den ihrer Familie zuarbeiten müssen, zuhause Hilfestellungen geben sollen oder auch Ceschwister versorgen [...]« (Bundeszentrale für politische Bildung/Robert Bosch Stiftung 2011, S. 29). 
eines ganzen Bündels von Faktoren erschwert. Die Jugendlichen fühlen sich oft selbst in vielerlei Hinsicht ausgegrenzt, verkennen ihre eigenen Potenziale und können sich selten in vollem Umfang persönlich entfalten. (Robert Bosch Stiftung 2013a, S. 5)

Auch in den Evaluationen zu DHDM und DmS wird das Thema soziale Ungleichheit aufgegriffen. ${ }^{156}$ So stellen Besand et al. Erklärungsmodelle aus der Forschungsliteratur zum >Phänomen Politikferneく zusammen (vgl. Besand/Fuhrmann o.J., S. 31-36; Besand/Birkenhauer/Lange 2013, S. 15-19). In prägnantester Kürze zusammengefasst lauten diese: $:^{157}$

a) es fehlt an Wissen bei den Jugendlichen,

b) Desinteresse der Jugendlichen,

c) es gibt bestimmte Schichten, die >politikfern « seien,

d) zunehmende Komplexität der Politik,

e) es besteht ein unsichtbares Politik-Interesse,

f) Politikferne als Kommunikationsproblem,

g) Politikferne als gesellschaftliche Exklusionsmechanismen.

Soziale Ungleichheit spielt in den Erklärungsmodellen z.B. in c) und g) eine Rolle (vgl. Besand/Fuhrmann o.J., S. 33-35). Aus diesen Konzepten und Erklärungen formen Besand/Fuhrmann eine Matrix mit pädagogischen und didaktischen Aufgaben, die als erste Bewertungsgrundlage für »Dialog macht Schule« (und später auch Besand/Birkenhauer/Lange für DHDM, vgl. dies. 2013, S. 15-19) gelten soll. In der Matrix kommt die (inhaltliche) Auseinandersetzung mit sozialer Ungleichheit jedoch kaum mehr vor, ${ }^{158}$ auch nicht bei DHDM (vgl. Besand/Birkenhauer/Lange 2013, S. 2of.). Bestenfalls noch unter dem Stichwort "genderbewusste Angebote« (Besand/Fuhrmann o.J., S. 36), was jedoch in den weiteren Ausführungen in der Evaluation keine Berücksichtigung mehr findet.

156 Vgl. auch Kapitel 2.2.1, in dem der von Besand et al. zusammengefasste Forschungsstand zur politischen Bildung mit >politikfernen Jugendlichen dargestellt wurde.

157 Ausführlich können diese in der veröffentlichten Evaluation zu »DU HAST DIE MACHT« nachgelesen werden - hier werden auch die Studien genannt, aufdie sich Besand/Birkenhauer/Lange beziehen (vgl. dies. 2013, S. 15-19).

158 In einer Grafik zu dieser Matrix werden die folgenden Kriterien genannt: »sich auf die Kommunikation einlassen «, »Konflikte aushalten«, »genderbewusste Angebote«, »Komplexität verringern«, »zwangslose Angebote«, »unkonventionelle Angebote«, »Artikulationsformen ernst nehmen«, »Wissen vermitteln«, »nicht nach Politik fragen«, »Politikersprech< entschlüsseln«, »hinter Desinteresse kein Unvermögen vermuten«, »Herausfordernde Zielgruppe annehmen«, »spannende und unterhaltsame Angebote schaffen«, »weites Politikverständnis anbieten« (Besand/Fuhrmann o.]., S. 36). 
Die Tatsache, dass soziale Ungleichheit zwar im Forschungsstand als Erklärung für die sogenannte >Politikferne` noch eine Rolle spielt, in der Matrix für die Konzeption der Bildungsangebote jedoch (als inhaltliches Thema) nicht mehr berücksichtigt wird, ist sicher nicht auf einen intentionalen Ausschluss zurückzuführen - wurde doch in dieser Untersuchung (vgl. Kapitel 2.2.3) gezeigt, dass im breiteren Fachdiskurs die Auseinandersetzung mit Ungleichheits- und Herrschaftsverhältnissen sowieso eine randständige Rolle spielt. Gleichzeitig ist damit auch ein Hinweis gegeben, wo die Innovationsfähigkeit und die Gemeinwohlorientierung der Programme ausbaufähig wären: In der Reflexion der Einbettung politischer Bildung in die Reproduktion von Herrschafts- und Ungleichheitsverhältnissen und in der Thematisierung von sozialen Ungleichheitsverhältnissen innerhalb der Programme. Dies dürfte in den nachfolgenden Ausführungen noch deutlicher werden.

Wie die Beschreibung der Formate (vgl. Kapitel 4.3.3.1 bis 4.3.3.3) zeigte, sollen >benachteiligte Jugendlichen den. Damit ist z.B. gemeint, dass jugendkulturelle Aspekte und Themen der Jugendlichen berücksichtigt werden. Bildungsangebote werden ansprechend gestaltet und sollen Spaß machen. Betrachtet man die formulierten Bildungsziele der Modellprojekte, so fällt auf, dass alle Programme die Jugendlichen stärken wollen - wie schon an der Strategie der RBS gezeigt wurde, also ein starker Fokus auf das Individuum gelegt wird. Bei DmS scheinen auch die schulischen Strukturen zumindest teilweise mit ins Blickfeld zu geraten:

»Dialog macht Schule will Demokratie in einer immer vielfältiger werdenden Cesellschaft stärken. Dem Selbstverständnis folgend, dass eine Demokratie von der Beteiligung aller gesellschaftlichen Cruppen lebt, wollen wir jungen Menschen aus sozial benachteiligten Verhältnissen das Cefühl vermitteln, dazuzugehören, gebraucht zu werden und ihnen das Rüstzeug an die Hand geben, die Gesellschaft von morgen mitzugestalten. Gleichzeitig wollen wir Schulen in sozialen Brennpunkten dabei unterstützen, sich auf eine immer multikultureller werdende Schülerschaft einzustellen, indem wir als fester Bestandteil des Schulalltags interkulturelle Brücken zwischen Schüler*innen und Lehrer*innen bauen.«(Dialog macht Schule 2017a)

DmS will die Jugendlichen erreichen, in dem es »ressourcen- und kompetenzorientiert « arbeite und

»[...] sowohl die psychosoziale Ebene der Jugendlichen berücksichtigt (Selbstvertrauen und Selbstwirksamkeit entwickeln) als auch Basiskompetenzen politischer Bildung (Urteils- und Handlungsfähigkeit sowie methodische Fähigkeiten) über einen längeren Zeitraum einübt und erprobt.« (Ebd.) ${ }^{159}$

159 Diese Aspekte spielten von Anfang an im Programm eine Rolle. So werden als drei von sechs Kernaspekten auch schon 2011 »Ressourcen- und Kompetenzorientierung«, »psychosoziale 
Für das Ziel einer Demokratie, »in der alle gesellschaftlichen Gruppen unabhängig von ihrer sozialen und kulturellen Herkunft, die Gesellschaft und Politik mitgestalten können« (dies. 2017c), sollen

»Schüler*innen [...] in ihrer demokratischen Bewusstseinsbildung gefördert werden und sich zu selbstbestimmen Persönlichkeiten entwickeln, die geleitet von ihren Stärken für sich und andere Verantwortung übernehmen.« (Ebd.) ${ }^{160}$

Der Fokus auf das Individuum wird auch im Programm »Lernort Stadion« deutlich. Als übergeordnetes Ziel wird in der Programmbeschreibung zunächst genannt, dass »Jugendliche [...] in ihrer Urteilsfähigkeit und ihrer Selbstverantwortung gestärkt [werden], um ihre Welt selbstbestimmter verändern zu können« (Robert Bosch Stiftung 2017m). In einer Auffächerung der Ziele wird zunächst sehr allgemein genannt, dass man die Jugendlichen »für politische Bildungsthemen und gesellschaftspolitische Fragestellungen interessieren « und »ihre sozialen Kompetenzen stärken « möchte (ebd.). ${ }^{161}$ Die Teilnehmenden sollen »motiviert werden« sich mit bestimmten Themen (»wie Gewalt, Rassismus, Homophobie, Diskriminierung und Vorurteilshandeln«) und »ihren persönlichen Rechten, Integrations- und Partizipationsmöglichkeiten auseinanderzusetzen« (ebd.). Der Kooperationspartner, die DFL Stiftung, schreibt auf seiner Website: »Wir möchten diese Jugendlichen >fit for life machen und sind überzeugt, dass sie trotzdem nicht unpolitisch sind es bedarf nur neuer Wege der politischen Bildung.« (Bundesliga Stiftung 2016c) ${ }^{162}$ Ziel sei es,

Ebene« und »Kompetenzentwicklung« genannt (Bundeszentrale für politische Bildung/Robert Bosch Stiftung 2011, S. 8). Die Evaluation des Programms zeigt auf, dass die Dialogmoderator*innen als Ziele ihrer Arbeit »Wissenssteigerung«, »|rritationen und produktive Brüche«, »Empathie, Perspektivenwechsel und Ambiguitätstoleranz« und »[a]rtikulationsfähig werden« benennen (Besand/Fuhrmann o.J., S. 104-106).

$160 \mathrm{Zu}$ diesen Bildungszielen, die darauf zielen, das Individuum zu stärken, passt, dass in dem jährlichen Weiterbildungsangebot für Dialogmoderator*innen (der »Vertiefungsakademie«) im Jahr 2016 u.a. ein Vortrag zur »positive[n] Psychologie« gehalten wurde, deren zentrale Fragen sei, »was Menschen stark macht und wie sie ihre Stärken entwickeln können« (Dialog macht Schule 2017e). Der Vortrag wurde von Viktoria Franz von der Universität Trier gehalten (vgl. ebd.).

161 Auch im Begleitheft (zur Methodensammlung) wird im direkten Anschluss an die Beschreibung der sozial prekären Lage der Jugendlichen betont, dass man deshalb die »sozialen Kernkompetenzen«stärken möchte (Robert Bosch Stiftung 2013a, S. 5).

162 Im sogenannten »Mission Statement« von »Lernort Stadion « wird ebenfalls das Ziel genannt, »[...] über den Fußball [...] Jugendliche sfit zu machen«, an der Cesellschaft aktiv und reflektiert teilzuhaben« (Robert Bosch Stiftung 2013a, S. 28). Man will sie »in ihrer Urteilsfähigkeit und Selbstverantwortung [...] stärken, damit sie ihre Welt selbstbestimmt gestalten können« (ebd.). 
»[...] Lern- und Denkanstöße zu geben, die bei den Jugendlichen Bildungsprozesse anregen und zu einer positiven, selbstverantwortlichen Entwicklung ihres persönlichen und beruflichen Lebensweges beitragen.« (Robert Bosch Stiftung 2013a, S. 5)

Hinsichtlich der "multiplen Problemlagen der Jugendlichen « wird betont, dass das Programm diese »nicht [...] lösen« könne, man jedoch das Empowerment der Jugendlichen fördern wolle: Das Programm

»kann im Sinne von sozialer Inklusion aktiv auf die Jugendlichen zugehen und ihnen eine positive, wertschätzende Lernerfahrung anbieten, die die Jugendlichen stärkt. Eine solche Lernerfahrung meint Empowerment. Sie zielt auf die Selbstbefähigung der Jugendlichen zur Persönlichkeitsentfaltung, zur besseren gesellschaftlichen Integration und zu gesellschaftlicher Partizipation. Lernort Stadion ermutigt die Jugendlichen dazu, ein demokratisches Miteinander im Projekt auszuprobieren, die eigene Rolle in der Gesellschaft zu reflektieren und Themen, die das Stadion bietet, kritisch zu betrachten, um hieraus Rückschlüsse für das eigene Tun zu ziehen.« (Ebd., S. 6)

Man will $\mathrm{zu}$ »solidarischem und emanzipiertem Denken und Handeln« ermutigen (ebd., S. 20). Das zugrunde gelegte politische Bildungsverständnis verweist darauf, dass davon ausgegangen wird, dass politische Beteiligung voraussetzungsreich ist und spezifischer Kompetenzen bedarf:

»Es geht um die Aktivierung der Jugendlichen zur Auseinandersetzung mit ihrem eigenen Lebensumfeld und zur selbstverantwortlichen Cestaltung des eigenen Lebens. Für die Bereitschaft, sich aktiv zu beteiligen, brauchen Jugendliche soziale, kommunikative, mediale und spezifische demokratische Kompetenzen.« (Ebd., S. 6) $)^{163}$

Die materiellen Voraussetzungen für demokratische Beteiligung - obwohl ihr Fehlen z.B. bei der Zielgruppenbeschreibung klar benannt wird - werden hier nicht mehr aufgeführt.

163 Dazu werden im Begleitheft »demokratische Basiskompetenzen« aufgezählt; im Vordergrund stünde die »Vermittlung von Toleranz und die Anerkennung der Menschenrechte« (Robert Bosch Stiftung 2013a, S. 7). Dass davon ausgegangen wird, dass die Teilnahme am politischen Leben voraussetzungsvoll ist, wird auch im zugrunde gelegten politischen Bildungsverständnis deutlich: „Unter politischer Bildung verstehen wir landläufig alle organisierten, kontinuierlichen und zielgerichteten pädagogischen Maßnahmen, um - in unserem Fall-jugendliche mit den zur Teilnahme am politischen und gesellschaftlichen Leben notwendigen Voraussetzungen auszustatten. Geleitet vom Gedanken der Demokratie ist es dabei Ziel der politischen Bildung, demokratische Spielregeln zu verankern und damit zur Herausbildung und Weiterentwicklung eines aktiven Cemeinwesens, gesellschaftlicher Partizipation und politischer Beteiligung möglichst aller Menschen beizutragen.« (ebd., S. 10) 
Auch im »Lernort Stadion« wird ein »weiter Politikbegriff« zugrunde gelegt dieser soll »[...] über Themen der eigenen Lebenswelt zu Inhalten und Strukturen des politischen Systems « führen (ebd.). Weiterhin heißt es: »Auf diese Weise können sich die Jugendlichen einbezogen fühlen und lernen, dass Politik mehr bedeutet als ein diffuses Gefühl der Ohnmacht, der Desorientierung, Überforderung und Intransparenz.« (Ebd.) Hierin deutet sich an, dass die Ursache für Ohnmachtserfahrungen und das Aufarbeiten dieser weniger im Mittelpunkt steht: Sondern es soll auf die bestehenden (eigenen) Machtressourcen aufmerksam gemacht werden - ganz ähnlich wie es Bröckling für die Machttheorie des Empowerments dargelegt hat (vgl. Kapitel 3.2.1). In diesem Sinne kann im Übrigen auch der Name des Programms »DU HAST DIE MACHT« gedeutet werden: Der Fokus liegt auf dem Entdecken der Machtquellen bei sich selbst - nicht bei der Analyse von gesellschaftlichen Bedingtheiten und Barrieren.

Die Angebote der einzelnen Lernzentren bei »Lernort Stadion« unterscheiden sich zwar, jedoch hat man übergreifende "Qualitätsmerkmale« entwickelt (vgl. Tabelle 3). Diese wurden im Rahmen von »Netzwerktreffen und Methodenwerkstätten« als »Standards für die politische Bildungsarbeit am Lernort Stadion« erarbeitet (Robert Bosch Stiftung 2017m).

\section{Tabelle 3: Qualitätsmerkmale Lernort Stadion}

\begin{tabular}{|l|l|}
\hline \multicolumn{2}{|l|}{ Qualitätsstandards des Modellprojekts Lernort Stadion } \\
\hline 1. & $\begin{array}{l}\text { Der politischen Bildung im Stadion liegt ein weit gefasster Politikbegriff zu- } \\
\text { grunde. Er meint nicht nur die Auseinandersetzung mit Systemen, Akteuren } \\
\text { und Entscheidungsprozessen, sondern nimmt die Beteiligungsmöglichkeiten } \\
\text { jedes Einzelnen in seinem Umfeld, zum Beispiel in Schule, Familie oder Freizeit, } \\
\text { in den Blick. Dadurch lernen Jugendliche, dass auch in ihrem Alltag eine politi- } \\
\text { sche Dimension steckt. }\end{array}$ \\
\hline 2. & $\begin{array}{l}\text { Gezielte und aufeinander abgestimmte Lernmodule stärken die individuellen } \\
\text { und gruppenbezogenen Kommunikations- und Handlungsfähigkeiten als de- } \\
\text { mokratische Basis-kompetenzen. Im Mittelpunkt stehen kritisches Denken, ei- } \\
\text { genverantwortliches Handeln, Solidarität, Toleranzfähigkeit, Respekt, Empa- } \\
\text { thie, das Aushalten von Widersprüchen und das gewaltfreie Lösen von Konflik- } \\
\text { ten. Hierzu ist ein ganzheitlicher Lernansatz notwendig. }\end{array}$ \\
\hline 3. & $\begin{array}{l}\text { Der politische Bildungsansatz im Lernort Stadion konzentriert sich auf den } \\
\text { Kernbereich »Demokratie Lernen«. Die Teilnehmer beschäftigen sich mit den } \\
\text { Werten und Ambivalenzen im Fußball und entwickeln darüber ein demokrati- } \\
\text { sches Bewusstsein. Ziel ist die Erkenntnis, dass Demokratie Lernen der Persön- } \\
\text { lichkeitsentwicklung dient und die eigene Rolle in der Cesellschaft stärkt. }\end{array}$ \\
\hline
\end{tabular}




\begin{tabular}{|c|c|}
\hline 4. & $\begin{array}{l}\text { Das Stadion dient als Anschauungsort für die gesellschaftspolitische Dimensi- } \\
\text { on der bearbeiteten Themen. So erkennen die Jugendlichen, dass sie sich mit } \\
\text { ihrem Umfeld aus unterschiedlichen Perspektiven auseinandersetzen können. }\end{array}$ \\
\hline 5. & $\begin{array}{l}\text { Das Konzept der politischen Bildung im Lernort Stadion schließt den Anspruch } \\
\text { auf gesellschaftliche Teilhabe ein. Es ist darauf ausgerichtet, Jugendliche zur } \\
\text { Partizipation - zum aktiven Mitgestalten des eigenen Umfelds - aufzufordern } \\
\text { und zu befähigen. }\end{array}$ \\
\hline 6. & $\begin{array}{l}\text { Die aktive Teilnahme an den Lernangeboten im Stadion ist für die Jugendlichen } \\
\text { grundsätzlich an Freiwilligkeit gebunden. Die Angebote zielen auf eine leben- } \\
\text { dige Diskussion, die zu reflektiertem Nachdenken ermutigt und Meinungsviel- } \\
\text { falt fördert. Eine Schlüsselrolle spielen die Authentizität und Qualifikation der } \\
\text { Referenten in den Lernzentren. }\end{array}$ \\
\hline 7. & $\begin{array}{l}\text { Die Faszination der Jugendlichen für Stadion und Fußballumfeld trifft auf einen } \\
\text { pädagogisch vorstrukturierten Lernort und projektorientierte Angebote, die } \\
\text { forschendes und selbstgesteuertes Lernen ermöglichen. Methoden des nicht } \\
\text { formalen Lernens bestärken die Jugendlichen darin, eine eigene Haltung zu } \\
\text { entwickeln und sich für eigene Ideen einzusetzen. }\end{array}$ \\
\hline 8. & $\begin{array}{l}\text { Lernprozess und Lernangebote sind an den wertschätzenden Grundprinzipi- } \\
\text { en der Anschauungs- und Erlebnispädagogik ausgerichtet. Der Lernansatz im } \\
\text { Lernort Stadion ist ressourcen- und subjektorientiert, um die sozialen Kompe- } \\
\text { tenzen der Jugendlichen zu stärken. }\end{array}$ \\
\hline 9. & $\begin{array}{l}\text { Komplexe gesellschaftspolitische Zusammenhänge werden nicht ausgeblen- } \\
\text { det, sondern durch niederschwellige, zunächst elementarisierte Zugänge über } \\
\text { Fußball und Sport begreifbar gemacht. Praktische, bewegungsreiche und inter- } \\
\text { aktive Module werden als » Türöffner den inhaltlichen Diskussions- und Refle- } \\
\text { xionsphasen vorangestellt. Sie ermöglichen neue Erfahrungen und schaffen die } \\
\text { nötige Offenheit und Motivation für das Demokratie Lernen. }\end{array}$ \\
\hline 10. & $\begin{array}{l}\text { Lernort Stadion schließt als einladender Lernort mit einer positiven und wert- } \\
\text { schätzenden Pädagogik an den Inklusionsgedanken an und kann gerade auch } \\
\text { benachteiligte Jugendliche motivieren und stärken. Damit wirkt der Ansatz } \\
\text { Ausgrenzungserfahrungen der Jugendlichen entgegen und baut Barrieren zur } \\
\text { gleichberechtigten Teilhabe an der Gesellschaft ab. }\end{array}$ \\
\hline
\end{tabular}

Quelle: Robert Bosch Stiftung 2017m, S. 26f. 
Die `Qualitätsmerkmale $<$ zeigen, dass alle Schlagworte und Konzepte aufgegriffen werden, die sich im Bildungsbereich aktuell großer Beliebtheit erfreuen: Partizipation, Inklusion, selbstgesteuertes Lernen, Erlebnispädagogik, Ressourcenorientierung usw. Die Auseinandersetzung mit sozialen Ungleichheits-, gesellschaftlichen Macht- und Herrschaftsverhältnissen ist jedoch kein Kriterium - sie mag in den Angeboten einzelner Lernzentren eine Rolle spielen, als Qualitätsmerkmal wurde sie nicht aufgenommen.

Eine Reflexion, wie zielgruppenadäquate Formate Ungleichheits- und Herrschaftsverhältnisse reproduzieren, soll an dieser Stelle nicht ausführlich erfolgen, sondern erst in Kapitel 5 auf Basis einer empirisch fundierten Fallstudie für das Programm »Micro Projects« vorgenommen werden (vgl. Kapitel 5.3). Dennoch sei an dieser Stelle ein Hinweis für weitere Analysen gegeben und ein kurze theoretische Überlegung erwähnt: Aus einer subjektkritischen Perspektive (die in Kapitel 3.2.1 dargelegt wurde) fällt auf, dass die Bedingtheit der Jugendlichen bzw. der Subjekte wenig thematisiert wird: Die Subjekte sollen demokratisches Bewusstsein ausbilden, eine eigene Haltung entwickeln, sich für ihre Ideen einsetzen, soziale Kompetenzen (weiter) ausbilden, es soll ihre Persönlichkeitsentwicklung und ihre Rolle in der Gesellschaft gestärkt werden (vgl. die Merkmale 3, 7, 8 in Tabelle 3). Der weite Politikbegriff wird hier so interpretiert, dass Beteiligungsmöglichkeiten im sozialen Umfeld identifiziert werden sollen (vgl. Merkmal 1 in Tabelle 3) - wie Beteiligungsmöglichkeiten gesellschaftlich eingeschränkt und bedingt sind, darauf liegt nicht der Fokus. Die Thematisierung von Ohnmacht, gesellschaftlichen Erwartungen und Zwängen, beschränkten oder ungleichen Beteiligungsmöglichkeiten - als »anti-heroisches Korrektiv«, wie es Bünger nennt (Bünger 2011, S. 323; vgl. Kapitel 3.2.1) - spielen somit keine Rolle. Somit kann argumentiert werden, dass auf diese Weise Ungleichheits- und Herrschaftsstrukturen reproduziert werden.

Dass soziale Ungleichheitsdimensionen wenig mitgedacht werden, zeigt ein weiterer Auszug aus dem Begleitheft zur Methodensammlung des Programms »Lernort Stadion«. Dort ist das Grundgesetz (GG) als wichtiger Bezugspunkt angeführt: Voraussetzung für Demokratie sei

»eine in der Verfassung garantierte rechtliche und politische Grundordnung, die allen Staatsbürgern bestimmte Grundrechte zusichert. Im Grundgesetz für die Bundesrepublik Deutschland (CC), das eine auf die Menschenwürde gegründete demokratische Ordnung schaffen und die Wiederkehr eines totalitären Systems verhindern will, stehen die wichtigsten Grundrechte bewusst ganz am Anfang des Verfassungstextes. (Robert Bosch Stiftung 2013a, S. 10)

Neben der Unantastbarkeit der Menschenwürde wird als ein weiteres Recht u.a. »allgemeine Handlungsfreiheit; Freiheit der Person; Recht auf Leben« genannt (ebd., S. 12). Es sei 
»die persönliche Entscheidung eines jeden Einzelnen, ob er in seiner Freizeit Sport treiben oder faul vor dem Fernsehapparat sitzen will, ob er Bayern München oder Holstein Kiel anfeuert, Tribünenkarten kauft oder lieber in der Fankurve steht - oder sich überhaupt nicht für Fußball interessiert. Beschränkt werden die Freiheitsrechte eines Menschen nur durch die Rechte anderer, das Sittengesetz und die verfassungsmäßige Ordnung, d.h. ein Fußballfan darf z.B. während einer Weltmeisterschaft sehr wohl seine Freunde zu einer Cartenparty mit Spielübertragung einladen, diese müssen aber Rücksicht auf die Nachbarn nehmen, falls jene sich vom Torjubel in ihrer Ruhe gestört fühlen.« (Ebd.)

Soziale Voraussetzung für demokratische Teilhabe werden hier nicht mitgedacht denn nicht jede* $r$ hat in der Gesellschaft die freie Entscheidung, woran er* sie teilnehmen kann. Mit der Rücksicht auf die Bedürfnisse von Anderen (Nachbar*innenBeispiel) wird eher eine sozialmoralische Erziehung angestrebt als politische Bildung. ${ }^{164}$

Dass soziale Ungleichheit nicht thematisiert wird, ist m.E. besonders bemerkenswert vor dem Hintergrund, dass man in einem politischen Bildungsprogramm, das sich an >benachteiligte Jugendliche richtet, (wohl leider) vielfältige Einschränkungen diskutieren könnte, die z.B. auf einen Mangel an ökonomischem Kapitel sowie der Wirksamkeit symbolischer Herrschaftsmechanismen (analog zu den Begriffen von Bourdieu, vgl. Kapitel 3.2.3) zurückzuführen sind.

Im Programm-Begleitheft für die Lernzentren wird als Bildungsziel auch mehrfach eine kritische Auseinandersetzung erwähnt: Man wolle »besonders sozial benachteiligte und bildungsferne Jugendliche [...] motivieren, sich am Beispiel Fußball kritisch mit ihrem Umfeld auseinanderzusetzen « (Robert Bosch Stiftung 2013a, S. 4), ${ }^{165}$ sie sollen »lernen, genau hinzusehen und Gegebenes kritisch zu hinterfragen « (ebd., S. 5). Auch wird z.B. im Kontext des GG-Bezugs betont, dass sich Fußball als Interpretationsfolie u.a. für die »Verletzbarkeit von Grundrechten« eigne (ebd., S. 11). Als Beispiel wird genannt, dass die Unantastbarkeit der Menschenwürde am Beispiel von »Beschimpfungen von Spielern und Schiedsrichtern im Stadion« diskutiert werden kann - »zumal, wenn beispielsweise rassistisch oder homophob motiviert« (ebd.).

164 Dies zeigt sich auch darin, wie die persönliche Entscheidungsfreiheit erläutert wird: „Gerade bei Jugendlichen erscheint es außerdem wichtig, auch die Tragweite der persönlichen Entscheidungsfreiheit und des persönlichen Handelns für das eigene Leben zu thematisieren, indem man etwa Jugendliche, die den Sport zum zentralen Freizeit- und Lebensinhalt erkoren haben, z.B. auf die Vernachlässigung von Freunden und Schule als mögliche negative Konsequenzen hinweist. « (Robert Bosch Stiftung 2013a, S. 12)

165 Das Zitat im Kontext lautet: »Ziel ist dabei, besonders sozial benachteiligte und bildungsferne Jugendliche darin zu bestärken, aktiv an der Cesellschaft teilzuhaben, demokratische Werthaltungen zu reflektieren, und sie zu motivieren, sich am Beispiel Fußball kritisch mit ihrem Umfeld auseinanderzusetzen.«(Robert Bosch Stiftung 2013a, S. 4) 
Um sich einen Eindruck von der Umsetzung des Programms zu verschaffen, wurde deshalb an einem mehrtägigen Workshop in einem Lernzentrum teilgenommen und im Nachgang ein Protokoll angefertigt (vgl. Gedächtnisprotokoll 2015). Hier ist zunächst positiv hervorzuheben, dass der Feldzugang problemlos möglich und das besuchte Lernzentrum sehr offen und unterstützend war. Auch für Kritik und Weiterentwicklung aus den Beobachtungen zeigte sich das pädagogische Personal sehr offen. Eine umfassende empirische Erhebung wurde ausgeschlossen, da das Programm aufgrund seines Alleinstellungsmerkmals >politische Bildung im Stadion werden mithilfe des Gedächtnisprotokolls (vgl. ebd.) dennoch drei Programmeinheiten kurz betrachtet, um damit zumindest verdeutlichen zu können, welches Potenzial die Untersuchung solcher Projekte mithilfe ethnografischer Ansätze hätte und dass solche Untersuchungen geeignet wären, um die Einbettung politischer Bildungsprogramme in Herrschafts- und Ungleichheitsverhältnisse $\mathrm{zu}$ reflektieren.

Ein Punkt, der nach der Analyse der Programmpublikationen offen blieb, war, ob und inwiefern die Grundrechte des GG in ihrem Spannungsverhältnis zur Realität behandelt werden? Dies geht aus den Programmbeschreibungen nicht klar hervor. In dem besuchten Workshop zum GG teilte das pädagogische Personal zunächst einen Lückentext zum GG aus. Der Text beinhaltete einzelne Sätze aus dem Verfassungstext, in denen einzelne Wörter fehlten. Die Jugendlichen mussten den Text in Gruppenarbeit ausfüllen, daraufhin im Plenum vergleichen. Eine kritische Einordnung des GG, z.B. in Hinblick auf die auch im Begleitheft von »Lernort Stadion« genannte >Verletzbarkeit von Grundrechten<, erfolgte nicht durch das pädagogische Personal. Im Gegenteil: Die Jugendlichen wurden gefragt, ob und wo sie schon einmal im Ausland im Urlaub gewesen seien. Als einige dann Russland oder Ägypten nannten, wurden sie gefragt, ob diese Länder auch ein GG haben. Die Jugendlichen verneinten, woraufhin das pädagogische Personal meinte, sie sollten doch froh sein, dass sie in einem freiheitlichen Land wie Deutschland leben.

In einer weiteren Workshop-Einheit, einem Besuch in einem Nachwuchsleistungszentrum, in dem Jugendliche zu professionellen Fußballspielern ausgebildet werden, war u.a. eine Gesprächsrunde der Teilnehmenden mit einem Jugendtrainer vorgesehen. Dieser stellte das Nachwuchsleistungszentrum vor und erwähnte in diesem Zuge auch die sozusagen freizeitlose Jugend von jungen Nachwuchssportler*innen, die de facto keine Zeit für Familie, Freund*innen oder eine*n feste*n Freund*in hätten. Es wurde erläutert, dass 2 von 100 Jugendlichen nach mehrjähriger Ausbildung vom Verein als Berufsfußballspieler*innen übernommen werden. Es findet also eine sehr starke Auslese statt, die Jugendlichen stehen in einem enormen Wettbewerb zueinander und werden angehalten, Höchstleistungen zu erbringen. Der Trainer erwähnte neben der Fußballbegeisterung der Jugendlichen 
auch, dass die psycho-soziale Beratungsstelle des Nachwuchsleistungszentrums ausgelastet sei.

Der Trainer problematisierte den Leistungsdruck nicht, was wenig überraschend ist, jedoch kamen auch vom pädagogischen Personal des Fanprojekts (das "Lernort Stadion« repräsentiert) keine kritischen Nachfragen. Die Darstellungen des Trainers, bzw. die darin deutlich werdende vorherrschende Leistungsideologie, blieben unhinterfragt. Stattdessen betonte die pädagogische Fachkraft in Hintergrundgesprächen mit mir immer wieder, dass sich die Jugendlichen (die gerade ihren Hauptschulabschluss nachholen und aus prekären Verhältnissen stammen) von den Jugendlichen aus dem Nachwuchsleistungszentrum >mal eine Scheibe abschneiden könnten<.

Die Auseinandersetzung mit der vorherrschenden Leistungsideologie anhand eines Leistungszentrums wäre als Lernanlass für politische Bildung geradezu ideal. Doch das pädagogische Personal scheint hierfür nicht sensibilisiert und betrachtet den disziplinierten bzw. auch disziplinierenden Alltag von Jugendlichen im Nachwuchsleistungszentrum eher im Rahmen einer Vorbildfunktion für andere Jugendliche, die zudem als weniger diszipliniert und leistungsstark betrachtet werden.

Bei der Konzeption der Stadionrundführungen wurde im Begleitheft zum Programm »Lernort Stadion« nicht ersichtlich, ob der Rundgang auch für die Auseinandersetzung mit sozialer Ungleichheit genutzt wird. Diese könnte im Stadion z.B. anhand unterschiedlicher Plätze für unterschiedliche soziale Gruppen (z.B. V.I.P.-Bereich, Fankurve etc.) diskutiert werden. In dem besuchten Workshop war dies nicht der Fall. Hier stellt sich auch die Frage, ob so eine Thematisierung und damit die Orientierung an einem weiten Politikbegriff, der auch Herrschafts-, Macht- und Ungleichheitsverhältnisse thematisiert, nicht auch den Interessen der DFL Stiftung entgegen liefe? Die DFL vermarktet schließlich Fußball (vgl. auch Ausführungen weiter unten) und profitiert somit von bestimmten Grenzziehungen im Stadion (z.B. V.I.P.-Bereich). Eine solche Thematisierung würde in gewisser Weise die eigene Geschäftsgrundlage infrage stellen.

Die Beobachtungen zeigen, dass ein weiter Politikbegriff ${ }^{166}$ und eine alltagsweltliche Orientierung - wie im Programm »Lernort Stadion«- nicht automatisch auch mit einer kritischen Demokratiebildung verbunden sein müssen, nämlich einer, die die sozialen Voraussetzungen von Demokratie und politischer Beteiligung in den Blick nimmt und für diese sensibilisiert ist.

Die drei Modellprojekte der RBS, die in Kooperation mit anderen Partnern wie der bpb oder den Fanprojekten durchgeführt werden, weisen mit den skizzierten Bildungszielen keinen unmittelbar ersichtlichen Bezug zu den Geschäftsfeldern

166 Der auch als sentgrenzter Politikbegriff<bezeichnet wird, vgl. dazu die Ausführungen in Kapitel 2.2.1. 
der Bosch $\mathrm{GmbH}$ auf. Wenn auch gleichzeitig die Vermittlung einer Weltanschauung deutlich wird, die bestimmte hegemoniale Muster reproduziert und auch im Kontext von Eigentümer*innen-Interessen interpretiert werden kann (zumindest in dem Sinne, dass sie diesen nicht entgegen läuft): z.B., wenn auf Aktivierung und Eigenverantwortung gesetzt wird, wenn der Fokus auf den sbenachteiligten Jugendlichen und nicht den benachteiligenden Strukturen liegt.

Jedoch, darauf sei hier abschließend verwiesen, sind im Programm »Lernort Stadion« durchaus auch Verbindungen zu Geschäftsinteressen erkenntlich; allerdings nicht zur Bosch GmbH, sondern zur DFL GmbH. Zur Erläuterung muss hier zunächst ein Blick auf die Stiftungsstrukturen der DFL Stiftung geworfen werden (vgl. Abb. 4). Die Stiftung wurde 2009 als Stiftung bürgerlichen Rechts vom Ligaverband e.V. und der DFL Deutsche Fußball Liga GmbH gegründet (vgl. Bundesliga Stiftung 2012, S. 6) ${ }^{167}$ Der Ligaverband (vgl. linke Grafik in Abb. 4: hier als »Die Liga Fußballverband e.V.« bezeichnet) ist ein »Zusammenschluss aller 36 lizensierten Vereine und Kapitalgesellschaften der Bundesliga und 2. Bundesliga« (Bundesliga Stiftung 2012, S. 12). Sie »beauftragt mit operativem Geschäft« wiederum die DFL als eine $\mathrm{GmbH}$ (ebd.). $\mathrm{Zu}$ den »Kernaufgaben« der DFL gehören die »Organisation und die Vermarktung des Profifußballs in Deutschland« (Bundesliga 2017). ${ }^{168}$

Damit ist die von vielen wahrscheinlich nur als Fußball- oder Sportstiftung wahrgenommene DFL Stiftung zwar keine klassische Unternehmensstiftung, da ihr Stifter nicht nur ein Unternehmen, sondern ein Unternehmen und ein Verein sind (vgl. linke Grafik bei Abb. 4). Da in dem Verein jedoch Kapitalgesellschaften organisiert sind und sich die DFL Stiftung zudem auch als CSR-Stiftung der DFL versteht, kann sie m.E. recht eindeutig als Unternehmensstiftung eingeordnet werden. Der Konstruktion als CSR-Stiftung entsprechend nimmt das Unternehmen DFL wesentlichen Einfluss auf die Stiftungsgremien: Die rechte Grafik in Abb. 4 zeigt, dass die beiden Stifter den Vorstand und Stiftungsrat bestellen.

167 Der Stiftungsstock wurde bei der Gründung bei einer Million festgesetzt (vgl. Bundesliga Stiftung 2012, S. 9f.). Die Mittelverwendung »speist sich« aus mehreren Quellen: Die Stiftung erhält die »Dividenden aus den Vermögensanlagen« und »Einzelspenden«, einen »Teil der im Rahmen der Sportgerichtsbarkeit ausgesprochenen Geldstrafen«, die »Vertragsstrafen, die im Rahmen der Lizenzierung anfallen« und die Erlöse aus den aller zwei Jahre stattfindendem »Benefiz-Länderspiel« (ebd., S. 10).

168 Außerdem ist sie »für die Ansetzung aller 612 Saisonspiele in der Bundesliga und 2. Bundesliga sowie die Ausrichtung der Relegationsspiele zuständig und gewährleistet durch ihre Arbeit einen reibungslosen, ausgewogenen und vor allem spannenden Wettbewerb im deutschen Profifußball« (Bundesliga 2017). Weiterhin gehört zum »Kerngeschäft der DFL [...] die Lizenzierung der 36 Proficlubs der Bundesliga und der 2. Bundesliga« (ebd.). 

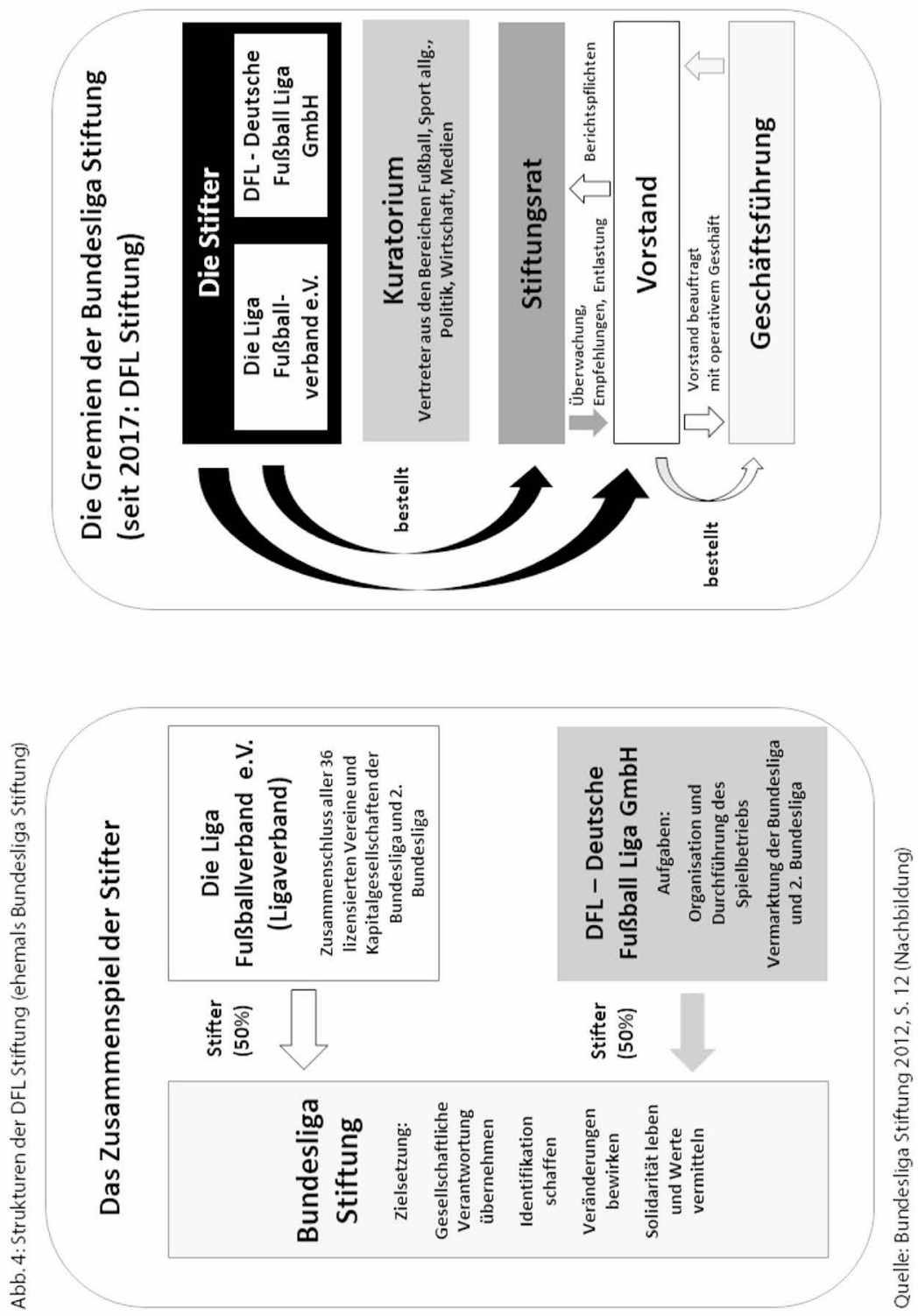
Bei der DFL Stiftung finden sich reichlich personelle Verflechtungen mit der DFL GmbH. So ist die DFL im höchsten Stiftungsgremium, dem zweiköpfigen Vorstand, ${ }^{169}$ vertreten: mit Jörg Degenhart, der zugleich "Direktor Finanzen, IT \& Organisation der DFL Deutsche Fußball Liga $\mathrm{GmbH}$ « ist (Bundesliga Stiftung 2017b). ${ }^{170}$ Der fünfköpfige Stiftungsrat besteht ausschließlich (!) aus Personen aus der DFL (aus ihrem Präsidium, ihrem Aufsichtsrat und ihrer Geschäftsführung). ${ }^{171}$ Er stellt neben dem Vorstand ein weiteres zentrales Gremium dar, denn er überwacht, berät und unterstützt den Stiftungsvorstand (vgl. dies. 2017a). ${ }^{172}$

Mit der Gründung der DFL Stiftung wurde das Ziel verbunden, »die gesellschaftliche Verantwortung des Profifußballs noch intensiver wahrzunehmen« (dies. 2017c). "Als eine führende Stiftung aus dem Sport für die Gesellschaft» hat die DFL Stiftung einen Schwerpunkt auf die Unterstützung von »Kinder[n], Menschen mit Behinderung und Sportler[n] anderer Sportarten « und das Thema Integration (dies. 2016a), ${ }^{173}$ in dessen Rahmen auch das Programm »Lernort Stadion « als eines der »Leuchtturmprojekte ${ }^{174}$ der Stiftung angesiedelt ist. Die Zusammenarbeit mit der DFL und den Clubs der Bundesliga und zweiten Bundesliga ist für die DFL Stiftung "von großer Bedeutung« (dies. 2012, S. 10). ${ }^{175} \mathrm{Um}$ »das enge Miteinander« mit den »Stiftern [...]« zu unterstreichen (DFL Stiftung 2017b), wurde die »Bundesliga Stiftung« 2017 sogar in »DFL Stiftung« umbenannt.

169 Der Vorstand sei »erstes Entscheidungsgremien« (Bundesliga Stiftung 2017a). Er »verwaltet die Stiftung. Er entwickelt ihre strategische Ausrichtung, stimmt sie mit dem Stiftungsrat ab und sorgt für ihre Umsetzung. «(Ebd.)

170 Der Vorstand besteht aus dem hauptamtlichen Vorstandsvorsitzenden Stefan Kiefer sowie Jörg Degenhart als stellvertretenden Vorstandsvorsitzenden (vgl. Bundesliga Stiftung 2017a).

171 Die Personalunionen scheinen in der Satzung festgelegt. Da die Satzung nicht veröffentlicht ist, kann dies jedoch nicht überprüft werden. Auf der Website findet sich folgender Verweis: »Den Stiftungsrat bilden DFL-Präsident Dr. Reinhard Rauball als Vorsitzender, dessen Stellvertreter Peter Peters (FC Schalke 04), und Fritz Keller (SC Freiburg) sowie der Vorsitzende der DFL-Geschäftsführung, Christian Seifert (stellvertretender Vorsitzender), und der Direktor Unternehmens- und Markenkommunikation der DFL, Christian Pfennig.«(Bundesliga Stiftung 2017a) Fritz Keller ist Aufsichtsratsmitglied der DFL (vgl. Bundesliga 2017).

172 Zudem fördern seine Mitglieder die Stiftungstätigkeit »ideell und leisten einen Beitrag für ein positives Erscheinungsbild der Stiftung« (Bundesliga Stiftung 2017a). Die Männer-Dominanz in Stiftungsgremien sticht generell ins Auge; bei der DFL Stiftung sind Stiftungsvorstand und -rat sogar ausschließlich mit Männern besetzt, im 18-köpfigen Kuratorium sind zwei Frauen vertreten (vgl. ebd.).

173 »Laut Satzung liegt der Stiftungszweck in der Förderung des Sports, der Bildung und Erziehung und der Völkerverständigung.«(Bundesliga Stiftung 2017c)

174 So die Aussage von Stefan Kiefer, dem Vorstandsvorsitzenden der Bundesliga-Stiftung (vgl. Robert Bosch Stiftung 2017m).

175 Die DFL Stiftung führt dazu aus: »Denn nur im engen Miteinander besitzen wir die Kraft, um unser gemeinsames Ziel zu erreichen: Chancen zu schaffen.«(Bundesliga Stiftung 2012, S. 10) 
Die Stiftung betont ausdrücklich, dass es Teil ihres Ansatzes sei, »neben der finanziellen Förderung die mediale und öffentliche Aufmerksamkeit, die der Fußball und seine Vorbilder zu bieten haben, für die geförderten Partner und Projekte zu nutzen« (Bundesliga Stiftung 2016a) und sich damit »für gemeinnützige Zwecke« (dies. 2016b) einzusetzen. Umgekehrt haben die Stifter - und damit auch die 36 Vereine und Kapitalgesellschaften der Bundesliga und 2. Bundesliga - natürlich auch etwas von der Stiftungsarbeit: »Das Ansehen der Bundesliga wächst schon seit Jahren in allen Gesellschaftsschichten. Daran trägt auch die Bundesliga Stiftung einen großen Anteil.« (dies. 2012, S. 36) ${ }^{176}$

Wenn die DFL Stiftung »Politische Bildung mit Fußballbezug « ${ }^{177}$ anbietet, ist das eben nicht nur einfach ein Bezug zu einer (Volks-)Sportart, sondern auch zum Produkt Fußball, dass die DFL vermarktet. So führt das Programm »Lernort Stadion « nicht nur potenzielle (und potenziell neue) Zuschauer*innen ins Stadion, sondern kann z.B. auch für die Imageaufbesserung von Stadien sorgen, von denen mittlerweile Besucher*innen (insbesondere Familien) aufgrund von gewalttätigen Ausschreitungen wegbleiben. Auch wird schließlich immer wieder diskutiert, ob die DFL für die teuren Polizeieinsätze bei Fußballspielen aufkommen soll. ${ }^{178}$ Die DFL will dies nicht, kann dafür aber mit Programmen wie »Lernort Stadion« öffentlich ihren Einsatz für Gewaltprävention demonstrieren. Das ist nicht per se verwerflich, verweist jedoch auf die Eigeninteressen der DFL, die sie über die Arbeit der DFL Stiftung realisieren kann.

\subsection{Zwischenfazit}

In diesem Kapitel wurden zunächst >unternehmensnahe Stiftungen akteure näher betrachtet: Hierbei ist zwischen Unternehmensstiftungen (auch: CSR-Stiftungen), die von Unternehmen gegründet und finanziell ausgestattet werden, und unternehmensverbundenen Stiftungen, die Anteile an Unternehmen halten, zu unterscheiden. Beide Formen der unternehmensnahen Stiftung erfüllen ökonomische und politische Funktionen und eröffnen z.B. über Personalunionen die Möglichkeit, Partikularinteressen umzusetzen (vgl. Kapitel 4.2.1). Der Typ der Unternehmensstiftung ist i.d.R. Teil der CSR-Strategien von Unternehmen

176 Diese Aussage trifft Christian Seifert in einem Interview im Tätigkeitsbericht der Stiftung aus dem Jahr 2012 - weshalb er von »Bundesliga Stiftung « und nicht »DFL Stiftung « spricht; er war der damalige stellvertretende Vorsitzende des Stiftungsrats (und gleichzeitige Vorsitzende der DFL-Geschäftsführung) (vgl. Bundesliga Stiftung 2012, S. 36).

177 So umschreibt die RBS das Programm (vgl. Robert Bosch Stiftung 2017m).

178 Vgl. dazu u.a. den Beitrag im hr-iNFO: »Wer zahlt? Streit um Polizeieinsätze beim Fußball« (hr-iNFO 2017). 
und insofern haben die Aktivitäten gerade dieser Stiftungen oftmals auch einen Bezug zum Kerngeschäft des Unternehmens (denn dies ist ein Merkmal von CSR). Insbesondere problematisch erscheinen die Partikularinteressen der unternehmensnahen, gemeinnützig anerkannten Stiftungen insofern, als dass sie sin Kombination gesetzlichen Transparenzstandards einhergehen.

Die darauffolgende explorative Analyse von unternehmensnahen Stiftungen in der politischen Bildung ergab, dass sich nicht nur eindeutig als unternehmensnah zu klassifizierende Stiftungen identifizieren lassen, sondern zahlreiche weitere Stiftungen, die nicht zu den klassischen unternehmensnahen Stiftungen gehören, jedoch vielfältige Verbindungen zu Unternehmen aufweisen - z.B. personeller und finanzieller Art, wenn in ihren Gremien zahlreiche Wirtschaftsvertreter*innen sitzen oder die Stiftungsmittel zu großen Teilen von Unternehmen stammen. Auch wurde deutlich, dass gerade bei diesen nicht eindeutig als unternehmensnah zu bestimmenden Stiftungen aufgrund der fehlenden Transparenz die genauen Verbindungen zu Unternehmen ohne größeren Rechercheaufwand (der hier nicht betrieben werden konnte) nicht zu identifizieren sind. So bleiben etwa Unternehmensverbundenheiten im Dunkeln, wenn sich Stiftungen weigern, die Zusammensetzung ihres Vermögens zu veröffentlichen. Die explorative Analyse der Aktivitäten der unternehmensnahen und der weiteren identifizierten Stiftungen, deren Unternehmensnähe als ungeklärt gelten muss, verweist auf ein vielfältiges Spektrum in der politischen Bildung hinsichtlich der umgesetzten Formate, Zielgruppen und Kooperationspartner.

Die nähere Betrachtung der RBS (in Kapitel 4.3) zeigte schließlich beispielhaft, wie komplex die Analyse der engen strukturellen und personellen Verflochtenheit der Stiftung mit dem Unternehmen ist und dass sich über die RBS als Teil eines sogenannten Doppelstiftungsmodells auch vielfältige Partikularinteressen verfolgen lassen: z.B. Steuerumgehung und die finanzielle Versorgung der Bosch-Familie.

Anhand eines Auszugs aus der nicht-veröffentlichten Strategie der RBS in ihrem Programmschwerpunkt »Jugend und Demokratie« konnte der Fokus auf sogenannte spolitikferne Zielgruppen ` der Stiftung in der politischen Bildung erläutert werden. Die drei Modellprojekte der Stiftung zeigen, dass neue Formate experimentell erprobt und begleitend evaluiert werden, um >benachteiligte Jugendliche mit politischen Bildungsangeboten zu erreichen - damit werden wichtige Innovationsbedarfe hinsichtlich der Formatgestaltung aufgegriffen. Hinsichtlich der Bildungsziele ist aus der in dieser Arbeit eingenommenen Theorieperspektive und auf Basis der Inhaltsanalyse von Programmbeschreibungen und Evaluationen jedoch festzuhalten, dass die soziale Benachteiligung der Jugendlichen in Programmbeschreibungen zwar benannt wird, jedoch innerhalb der Programme keine Auseinandersetzung mit sozialen Ungleichheitsverhältnissen und den sozialen Vorausset- 
zungen fokussiert wird. Jugendliche sollen vor allem als Individuen gestärkt werden, da ein wichtiges Ziel ist, dass sie >Eigenverantwortung übernehmen.

Schließlich wurde auch deutlich, dass in den Modellprojekten der RBS keine unmittelbaren Bezüge zu den Geschäftsfeldern der Bosch $\mathrm{GmbH}$ bestehen - wenn auch die Programme im Interesse mittelbarer Eigentümer*innen-Interessen gedeutet werden können, wie die Förderung von >Eigenverantwortungく zeigt. Das Programm "Lernort Stadion«, das 'politische Bildung mit Fußballbezug anbietet und bei dem die RBS mit der DFL Stiftung kooperiert, weist dagegen einen Bezug zu den Geschäftsinteressen der DFL auf. Bei dem Programm »Lernort Stadion" wirken jedoch noch weitere Partner mit, wie die Fanprojekte. Der Umstand, dass CSR allgemein und so auch CSR-Stiftungen einen Bezug zum Kerngeschäft von Unternehmen haben (vgl. Kapitel 4.1.1, 4.2.1), gleichzeitig jedoch CSR-Programme mit unterschiedlichen zivilgesellschaftlichen Kooperationspartnern durchgeführt werden (wie z.B. den `Fanprojekten « im »Lernort Stadion«), wirft viele Fragen für die politische Bildung auf: Beispielsweise, welche Prioritäten bestimmter Partner wie durchgesetzt werden. Nachfolgend wird deshalb ein Engagementprogramm einer CSR-Stiftung, das sich an >benachteiligte Jugendliche< richtet und mit Partnern aus der Jugendarbeit umgesetzt wird, im Rahmen einer Fallstudie untersucht. 
